CIRJE-F-1052

\title{
Trading and Ordering Patterns of Market Participants in High Frequency Trading Environment -Empirical Study in the Japanese Stock Market-
}

\author{
Taiga Saito \\ The University of Tokyo \\ Takanori Adachi \\ Ritsumeikan University \\ Teruo Nakatsuma \\ Keio University \\ Akihiko Takahashi \\ The University of Tokyo \\ Hiroshi Tsuda \\ Doshisha University \\ Naoyuki Yoshino \\ ADBI Institute
}

June 2017

CIRJE Discussion Papers can be downloaded without charge from:

http://www.cirje.e.u-tokyo.ac.jp/research/03research02dp.html

Discussion Papers are a series of manuscripts in their draft form. They are not intended for circulation or distribution except as indicated by the author. For that reason Discussion Papers may not be reproduced or distributed without the written consent of the author. 


\title{
Trading and Ordering Patterns of Market Participants in High Frequency Trading Environment -Empirical Study in the Japanese Stock Market- *
}

Taiga Saito $\dagger$ Takanori Adachi $\ddagger$ Teruo Nakatsuma $§$ Akihiko Takahashi $₫$ Hiroshi Tsuda I, Naoyuki Yoshino **

\begin{abstract}
In this study, we investigate ordering patterns of different types of market participants in Tokyo Stock Exchange (TSE) by examining order records of the listed stocks. Firstly, we categorize the virtual servers in the trading system of TSE, each of which is linked to a single trading participant, by the ratio of cancellation and execution in the order placement as well as the number of executions at the opening of the afternoon session. Then, we analyze ordering patterns of the servers in the categories in short intervals for the top 10 highest trading volume stocks. By classifying the intervals into four cases by returns, we observe how different types of market participants submit or execute orders in the market situations. Moreover, we investigate the shares of the executed volumes for the different types of servers in the swings and roundabouts of the Nikkei 225 index, which were observed in July, August, and September in 2015. The main findings of this study are as follows: Server type A, which supposedly includes non-market making proprietary traders with high-speed algorithmic strategies, executes and places orders along with the direction of the market. The shares of the execution and order volumes along with the market direction increase when the stock price moves sharply. Server type B, which presumably includes servers employing a market making strategy with high cancellation and low execution ratio, shifts its market making price ranges in the rapid price movements. We observe that passive servers in Server type B have a large share and buy at low levels in the price falls. Also, Server type B, as well as Server type A, makes profit in the price falling days and particularly, the aggressive servers in the server type make most of the profit. Server type C, which is assumed to include servers receiving orders from small investors, constantly has a large share of execution and order volume.
\end{abstract}

*All the contents expressed in this research are solely those of the authors and do not represent any views or opinions of any institutions. In particular, they are not an official view of the Financial Services Agency or FSA Institute. The authors are not responsible or liable in any manner for any losses and/or damages caused by the use of any contents in this research.

${ }^{\dagger}$ Graduate School of Econo mics, University of Tokyo. Former research fellow at Financial Research Center in Financial Services Agency, Government of Japan. saitotaiga@hotmail.com

${ }^{\ddagger}$ BKC Research Organization of Social Sciences, Ritsumeikan University.

$\S$ Department of Economics, Keio University

"Graduate School of Economics, University of Tokyo

"Department of Mathematical Sciences, Doshisha University.

**Financial Services Agency, Government of Japan. ADBI Institute. 


\section{Introduction}

High frequency trading environment has become common globally because of the advancements in information technology in the financial industry. While this enables millisecond high-speed trading of traders with algorithmic strategies, highly volatile intraday price movements, represented by the Flash Crash in 2010 in the U.S. stock market, have been observed in financial markets. In Japan, since Arrowhead, the high-speed execution system, was first introduced in 2010 and especially the tick sizes were narrowed in 2014 in TSE, we have come to observe large intraday stock price movements. Since excessively volatile markets are undesirable for investor protection, identifying the cause of the price instability is important from the regulatory perspective.

Although it has been said that this price instability in the high-speed trading environment might be caused by the high frequency traders (HFTs), the mechanisms are yet unknown. Following studies investigate HFTs' trading behaviors and their impacts on stock markets: Riordan and Storkenmaier [1] explores the relation between the latency of trading and the price discovery by using the data of DAX. Menkveld [2] analyzes HFTs' trading strategies across two different stock markets. O' Hara [3] discusses effects of the high frequency trading environment on the financial market including trading strategies of the market participants. Kirilenko et al. [4] examines HFTs' trading patterns in the flash crash, which took place on 6th May 2010, with E-mini S\&P futures contract data. Boehmer et al. [5] analyzes influences of the short-selling banning in 2008 on the market activities. Hasbrouck and Saar [6] investigates impacts of the low-latency trading on the market qualities using NASDAQ data. Carrion [7] examines trading performances of HFTs and their influences on the market with the NASDAQ data. Brogaard et al. [8] explores relations between HFTs' trading volumes and the stock returns in NASDAQ by the use of the state space model. Particularly, it decomposes the stock return into the overshoot and price efficiency effects, and examines the relation between these effects and the trading volumes of HFTs. Hosaka [9] analyzes HFTs' trading and ordering patterns and their impacts in TSE comparing those of the other servers, and concludes that HFTs provide liquidities in the market and help stabilize the stock prices.

On the other hand, our study investigates trading strategies and ordering patterns of four different types of market players, one of which presumably includes non-market making proprietary traders with high-speed algorithmic strategies, in TSE. This focuses on the order placement/cancellation and execution patterns in the short periods of different market situations using not only executed records, but also limit/cancellation/modification orders submitted. This study provides new insights on what is happening in the price movements; who are trading and submitting what type of orders, especially for traders with non-market making strategies.

In particular, we take the following procedure. First, we examine ordering and trading patterns of each ID of virtual servers, which is linked to a single market participant, by using order data of stocks listed in TSE. In detail, we calculate the following ratios for all the server IDs with all the order data of listed stocks as of 8th July 2015 when the Nikkei 225 index declined by 2.69\%: cancellation and execution ratio, number of executions at the opening of the afternoon session, and the average volume per order. After classifying the server IDs based on the ratios into four groups, we investigate executed volumes and changes in volume of orders in 10 second periods for the top 10 large trading volume 
stocks, which we consider to be representatives of the price movements of the index, in four different market situations depending on the return in the short intervals; price decline more than $0.10 \%$, price decline less than $0.10 \%$, price increase less than $0.10 \%$, and price increase more than $0.10 \%$.

Moreover, we analyze the large stock price movements in the swings and roundabouts of the Nikkei 225 index, which occurred in July, August, and September 2015, by examining the shares of the executed volume for the top 10 large trading volume stocks for the four server types. In detail, we classify the 10 second intervals by the return and the thinness of the orders, which is defined as the return divided by the executed volume in the Japanese yen (JPY), and observe the shares of the selling and buying executed volumes for the four server types.

The main findings of this study are as follows. Server type A, which supposedly includes non-market making proprietary traders with high-speed algorithmic strategies, executes and places orders along with the market direction. In particular, the share of trading volume as well as the order volume along with the market direction increases as the stock price moves sharply. Server type B, which presumably includes servers employing a market making strategy with high cancellation and low execution ratio, shifts its market making price ranges in the rapid price movements. Server type C, which is assumed to contain servers receiving orders from small investors, constantly has a large share of execution and order volume regardless of the market situations. In the market falls of the swings and roundabouts for the Nikkei 225 index, Server type A has large shares of selling volumes for the 10 representative stocks. In the days of index rebound, two patterns are observed. The first one is that the market is highly liquid and Server type $\mathrm{C}$ has the largest share of buying volume in the price rises. The second one is that the market is thin and Server type A has a high share of buying volume in the price rises as well as selling volume in the price falls. Also, in a quiet market where most of the price movements are small, we observe similar trading patterns to the days in the swings and roundabouts for each server type.

Furthermore, we investigate at which level Server type B buys in the price falls and how much P\&L this server type as well as Server type A makes in price falling days. We observe that passive servers in Server type B have a large share and buy at low levels in the price falls. Also, Server type B as well as Server type A makes profit in the price falling days and particularly, the aggressive servers in the server type make most of the profit.

This paper is organized as follows. Section 2 explains the details of the data provided by Japan Exchange Group (JPX) and the methodology to classify the server IDs into the four categories depending on the ratios which represent the features of trading or ordering patterns. Section 3 presents analyses of the trading and ordering patterns of the server types in the swing and the roundabouts as well as in a quiet market. Section 4 investigates the price levels where Server type B buys in the price falls and how much profit this server type as well as Server type A makes. Finally, Section 5 concludes. 


\section{Methodology}

\subsection{Data set}

We use order data of all the listed stocks in TSE on 8th July 2015 for the classification of server IDs. The details of the data set are as follows. All the order records are labeled with either of the following types:

1. new order,

2. executed order,

3. order modification canceling an old status,

4. order modification with a new status,

5. canceled order,

6. expired order.

Moreover, each order record accompanies the following information: order volume, buy or sell, order price, time stamp, order ID, server ID and last traded price. First, let us fix the ticker symbol of the stock $k$ and the trading date. Let $N^{(k)}$ be the number of all orders for the stock in the day. For the $i$-th order $\left(1 \leq i \leq N^{(k)}\right)$ of the day for the stock, we define a collection of the information $\mathbf{x}_{i}=\left(x_{i 1}^{(k)}, x_{i 2}^{(k)}, x_{i 3}^{(k)}, x_{i 4}^{(k)}, x_{i 5}^{(k)}, x_{i 6}^{(k)}, x_{i 7}^{(k)}, x_{i 8}^{(k)}\right)$, where $x_{i 1}^{(k)} \in\{1,2,3,4,5,6,7\}, x_{i 2}^{(k)} \in \mathbf{R}, x_{i 3}^{(k)} \in\{0,1\}, x_{i 4}^{(k)} \in(0, \infty), x_{i 5}^{(k)} \in[0, T], x_{i 6}^{(k)} \in \mathcal{O}$, $x_{i 7}^{(k)} \in \mathcal{S}, x_{i 8}^{(k)} \in \mathbf{R}$. Here, $\mathcal{O}$ is the set of the order IDs and $\mathcal{S}$ is the set of the server IDs. $x_{i 1}^{(k)}, x_{i 2}^{(k)}, x_{i 3}^{(k)}, x_{i 4}^{(k)}, x_{i 5}^{(k)}, x_{i 6}^{(k)}, x_{i 7}^{(k)}$ and $x_{i 8}^{(k)}$ represent the order status, the order volume, buy or sell, the order price, the time stamp, the order ID, the server ID and the previous price, respectively. $x_{i, 2}^{(k)}$, the order volume, takes a positive value if it is a new order or an order modification with a new status, and a negative value if it is a cancellation order or an order modification canceling an old status. $x_{i, 3}^{(k)}$ is 0 for a buying order and 1 for a selling order. The times 0 and $T$ correspond to 9:00:00 a.m. and 3:00:00 p.m. JST, respectively, which are the opening and closing times of the Tokyo stock market.

\subsubsection{Remarks on server IDs and limitation of the analysis}

Server IDs are the IDs allocated to the virtual servers used for the connections between the trading participants and TSE in the trading system. The participants, who serve as brokers between TSE and their clients, are the registered securities companies in TSE. They own multiple virtual servers, each of which has a limit in trading volume and the server IDs do not overlap among different participants. Generally, orders from different customers of a participant are submitted to TSE through one server ID. However, some server IDs of participants are kept for exclusive use of their special customers, such as hedge funds trading with algorithmic strategies.

Another important point to notice is that participants can specify a server ID in their submission of orders, which means that they can use different server IDs for new submission, modification, and cancellation of a single order whose life is tracked by an order ID. Hence, the Server IDs do not perfectly reflect the behavior of the final investors. 
One possible way to capture the behavior of the final investors better is that we bundle the server IDs used for the same order ID and assume a correspondence relation between the bundles and the final investors. However, we focus on server ID based analysis due to the burden of computation.

\subsection{Cancellation and execution ratio of servers}

Next, we define a cancellation and an execution ratio of servers based on order data on all listed stocks in TSE on 8th July 2015. We have chosen the day when the Nikkei 225 stock index fell significantly, more than $3 \%$ from the closing level of the previous day. This is the fifth largest price decline in absolute index value in 2015. In particular, the prices of the Nikkei 225 future contracts dropped significantly in response to the large drop in the Shanghai market, during the lunch break of the underlying stock market. Consequently, large numbers of limit sell orders were placed during the lunch break and the most prices of the stocks opened at lower levels at the beginning of the afternoon session.

Let $c_{s}$ and $h_{s}$ be the cancellation and the execution ratio, respectively. We divide the market opening periods, which are from 9:00 a.m. to 11:30 a.m. and from 0:30 p.m. to 3:00 p.m., into 1,800 ten-second intervals. Note that the interval from 11:30 a.m. to 0:30 p.m. is the lunch break when the underlying stock market is closed.

We define them as follows.

$$
\begin{array}{r}
c_{s}=\frac{\sum_{k \in K} \sum_{i=1}^{N^{(k)}} 1_{\left\{x_{i 1}^{(k)}=5, x_{i 7}^{(k)}=s\right\}}}{\sum_{k \in K} \sum_{i=1}^{N^{(k)}} 1_{\left\{x_{i 1}^{(k)}=1, x_{i 7}^{(k)}=s\right\}}}, \\
h_{s}=\frac{\sum_{k \in K} \sum_{i=1}^{N^{(k)}} 1_{\left\{x_{i 1}^{(k)}=2, x_{i 7}^{(k)}=s\right\}}}{\sum_{k \in K} \sum_{i=1}^{N^{(k)}} 1_{\left\{x_{i 1}^{(k)}=1, x_{i 7}^{(k)}=s\right\}}},
\end{array}
$$

where $K$ is the set of all the listed stocks in TSE. $c_{s}$, the cancellation ratio of the server $s$, is the number of cancellation orders from the server $s$ divided by the number of new orders submitted by $s$. Similarly, $h_{s}$, the hit ratio of the server $s$, is the number of executed orders from the server $s$ divided by the number of new orders from $s$.

Let $\mathrm{C} 1, \mathrm{C} 2, \mathrm{C} 3, \mathrm{C} 4$ and $\mathrm{C} 5$ be the categories of the servers with the cancellation ratio $0-20 \%, 20-40 \%, 40-60 \%, 60-80 \%$, and 80-100\%, respectively. Similarly, let H1, H2, $\mathrm{H} 3$, and $\mathrm{H} 4$ be the categories with the executed ratio $0-25 \%, 25-50 \%, 50-75 \%, 75-100 \%$, respectively. Table 1 summarizes the numbers and shares of the new, cancellation and executed orders by server type. 


\begin{tabular}{|c|c|c|c|c|c|c|c|c|c|}
\hline & & \# of servers & Share & \# of new orders & Share & \# of canceled orders & Share & \# of executed orders & Share \\
\hline $\mathrm{C} 1$ & $\mathrm{H} 1$ & 143 & $3 \%$ & $1,915,078$ & $6 \%$ & 316 & $0 \%$ & 133,418 & $2 \%$ \\
\hline $\mathrm{C} 1$ & $\mathrm{H} 2$ & 135 & $3 \%$ & 427,087 & $1 \%$ & 9,878 & $0 \%$ & 146,506 & $2 \%$ \\
\hline $\mathrm{C} 1$ & H3 & 456 & $10 \%$ & 826,931 & $2 \%$ & 120,702 & $1 \%$ & 488,436 & $6 \%$ \\
\hline $\mathrm{C} 1$ & $\mathrm{H} 4$ & 316 & $7 \%$ & 401,839 & $1 \%$ & 58,885 & $0 \%$ & 382,960 & $5 \%$ \\
\hline $\mathrm{C}_{2}$ & $\mathrm{H} 1$ & 13 & $0 \%$ & 49,213 & $0 \%$ & 14,609 & $0 \%$ & 7,519 & $0 \%$ \\
\hline $\mathrm{C} 2$ & $\mathrm{H} 2$ & 32 & $1 \%$ & 125,180 & $0 \%$ & 40,959 & $0 \%$ & 58,010 & $1 \%$ \\
\hline $\mathrm{C}^{2}$ & H3 & 214 & $5 \%$ & $4,023,461$ & $12 \%$ & $1,189,379$ & $5 \%$ & $2,298,612$ & $27 \%$ \\
\hline $\mathrm{C} 2$ & $\mathrm{H} 4$ & 354 & $8 \%$ & 288,256 & $1 \%$ & 86,081 & $0 \%$ & 268,172 & $3 \%$ \\
\hline $\mathrm{C}$ & $\mathrm{H} 1$ & 23 & $1 \%$ & 49,941 & $0 \%$ & 21,227 & $0 \%$ & 9,252 & $0 \%$ \\
\hline $\mathrm{C}$ & $\mathrm{H} 2$ & 489 & $11 \%$ & $2,429,534$ & $7 \%$ & $1,351,270$ & $6 \%$ & 946,029 & $11 \%$ \\
\hline $\mathrm{C}$ & H3 & 206 & $5 \%$ & 676,088 & $2 \%$ & 343,740 & $1 \%$ & 398,531 & $5 \%$ \\
\hline $\mathrm{C}$ & $\mathrm{H} 4$ & 23 & $1 \%$ & 35,019 & $0 \%$ & 14,971 & $0 \%$ & 34,486 & $0 \%$ \\
\hline $\mathrm{C} 4$ & H1 & 195 & $4 \%$ & $2,331,722$ & $7 \%$ & $1,754,429$ & $7 \%$ & 477,943 & $6 \%$ \\
\hline $\mathrm{C} 4$ & $\mathrm{H} 2$ & 467 & $10 \%$ & $3,811,269$ & $11 \%$ & $2,755,218$ & $12 \%$ & $1,353,687$ & $16 \%$ \\
\hline $\mathrm{C} 4$ & H3 & 61 & $1 \%$ & 326,843 & $1 \%$ & 210,407 & $1 \%$ & 177,588 & $2 \%$ \\
\hline $\mathrm{C} 4$ & $\mathrm{H} 4$ & 5 & $0 \%$ & 6,599 & $0 \%$ & 4,458 & $0 \%$ & 5,323 & $0 \%$ \\
\hline $\mathrm{C} 5$ & $\mathrm{H} 1$ & 894 & $20 \%$ & $15,773,284$ & $46 \%$ & $14,943,880$ & $64 \%$ & $1,034,581$ & $12 \%$ \\
\hline $\mathrm{Cs}$ & $\mathrm{H} 2$ & 115 & $3 \%$ & 556,794 & $2 \%$ & 488,690 & $2 \%$ & 172,628 & $2 \%$ \\
\hline $\mathrm{C} 5$ & H3 & 5 & $0 \%$ & 12,355 & $0 \%$ & 14,442 & $0 \%$ & 8,233 & $0 \%$ \\
\hline $\mathrm{C} 5$ & $\mathrm{H} 4$ & 0 & $0 \%$ & 0 & $0 \%$ & 0 & $0 \%$ & 0 & $0 \%$ \\
\hline & Total & 4,448 & $100 \%$ & $34,075,892$ & $100 \%$ & $23,447,976$ & $100 \%$ & $8,408,934$ & $100 \%$ \\
\hline
\end{tabular}

Table 1: Distribution of the new, cancellation and executed orders for all listed stocks as of 8th July 2015 by server type.

We observe that in total, $69 \%$ of the new orders are canceled and $25 \%$ of them are executed. The rest are orders expired either as the special orders such as the immediate or cancel orders or at the end of the trading session of the day. Particularly, C5H1, the category with the highest cancellation and the lowest executed ratios which accounts for $20 \%$ of the servers in number, has $46 \%$ share of the new orders and $64 \%$ share of the cancellation orders. It is notable that although only $7 \%$ of the new orders submitted by C5H1 are executed, they still consist of $10 \%$ of all the executed orders.

\subsection{Classification of server types}

Next, we categorize the servers, which have more than 100 new order submissions in the day, into four types. Firstly, following Hosaka [9] for the definition of HFTs, we categorize the server IDs labeled C5H1, C4H1, C3H1 and C2H1 as Server type B. Then, for the other server IDs, if a server does not have any sell orders executed in the one second after the opening of the afternoon session, when the most of the executed orders are as a result of participating in Itayose in the lunch break, then we regard it as the server in Server type A. Here, Itayose is a method of matching orders submitted in the lunch break or the pre-opening of the market, which is adopted in TSE. In the method, only the price level of orders matters for the order matching, and the price-time priority principle does not apply.

Since it is expected that servers dealing with customer order flows place at least one sell order at a low price when there is a negative market surprise in the lunch break, we presume that Server type A includes servers of proprietary traders with non-market making and high-speed algorithmic trading strategies. Moreover, for the servers in neither Server type A nor Server type B, if the average volume in JPY per order is less than JPY 3 million, we label them as Server type C, otherwise Server type D. Here, we define the average volume per order as follows. For each new order of server IDs, we multiply the 
order volume by the order price. We take the average over the new orders submitted by the server ID.

Server type B is expected to include traders who take a market making strategy actively placing orders at the best bid or offer. Server type $\mathrm{C}$ is supposed to include securities companies who mainly deal with orders from retail customers. Server type D is assumed to include the servers of securities companies which primarily deal with large orders from their customers. We note that traders, who execute large orders from institutional investors such as insurance companies and pensions with algorithmic strategies, are assumed to belong to Server type C or D, since they also make use of Itayose in the lunch break and the pre-opening period. Also, non-market-making proprietary traders with algorithmic strategies using Itayose are expected to belong to Server type C or D.

\begin{tabular}{|l|cc|cc|ccc|}
\hline & \# of servers & Share & \# of new orders & Share & \# of cancellation orders & Cancellation ratio & Share \\
\hline Server type A & 1,633 & $37 \%$ & $2,585,173$ & $8 \%$ & 943,715 & $37 \%$ & $4 \%$ \\
Server type B & 1,125 & $25 \%$ & $18,204,160$ & $53 \%$ & $16,734,145$ & $92 \%$ & $71 \%$ \\
Server type C & 1,115 & $25 \%$ & $11,300,943$ & $33 \%$ & $4,921,759$ & $44 \%$ & $21 \%$ \\
Server type D & 273 & $6 \%$ & $1,976,217$ & $6 \%$ & 823,922 & $42 \%$ & $4 \%$ \\
\hline
\end{tabular}

Table 2: Number of servers, new orders, and cancellation orders by server type

\begin{tabular}{|l|ccc|c|}
\hline & \# of executed orders & Execution ratio & Share & Average volume per order in JPY \\
\hline Server type A & $1,014,443$ & $39 \%$ & $12 \%$ & $3,784,789$ \\
Server type B & $1,529,295$ & $8 \%$ & $18 \%$ & $2,581,234$ \\
Server type C & $4,825,364$ & $43 \%$ & $57 \%$ & $1,024,046$ \\
Server type D & $1,032,812$ & $52 \%$ & $12 \%$ & $8,315,842$ \\
\hline
\end{tabular}

Table 3: Number of executed orders by server type

Tables 2 and 3 show numbers of servers, new orders, cancellation orders, and executed orders, those ratios against the new orders, and average volumes in JPY per order by server type. We observe that Server type A has the largest number of server IDs, 37\% of the total numbers, though the shares of the new, executed, and cancellation orders are low. Server type B has the largest shares of the new orders and the cancellation orders, which are $53 \%$ and $71 \%$, respectively, and $18 \%$ share of the executed orders, which is the second largest after that of Server type C. Server type C has the largest share of the executed orders, $57 \%$, and the second largest shares of the new and cancellation orders and the number of server IDs. Server type D has the highest execution ratio, $52 \%$, and the highest order volume per order, which is around JPY 8 million, although the shares of the server numbers and the new, cancellation, executed orders are the lowest among the four categories.

\begin{tabular}{|c|cccc|}
\hline & H1 & H2 & H3 & H4 \\
\hline C1 & 128 & 121 & 380 & 186 \\
C2 & 0 & 18 & 30 & 246 \\
C3 & 0 & 357 & 59 & 5 \\
C4 & 0 & 70 & 12 & 3 \\
C5 & 0 & 17 & 1 & 0 \\
\hline
\end{tabular}

Table 4: Server distribution, Server type A 


\begin{tabular}{|c|cccc|}
\hline & H1 & H2 & H3 & H4 \\
\hline C1 & 0 & 0 & 0 & 0 \\
C2 & 13 & 0 & 0 & 0 \\
C3 & 23 & 0 & 0 & 0 \\
C4 & 195 & 0 & 0 & 0 \\
C5 & 894 & 0 & 0 & 0 \\
\hline
\end{tabular}

Table 5: Server distribution, Server type B

\begin{tabular}{|c|cccc|}
\hline & H1 & H2 & H3 & H4 \\
\hline C1 & 5 & 14 & 64 & 110 \\
C2 & 0 & 9 & 151 & 59 \\
C3 & 0 & 129 & 126 & 9 \\
C4 & 0 & 345 & 40 & 2 \\
C5 & 0 & 48 & 4 & 0 \\
\hline
\end{tabular}

Table 6: Server distribution, Server type C

\begin{tabular}{|c|cccc|}
\hline & H1 & H2 & H3 & H4 \\
\hline C1 & 10 & 0 & 12 & 20 \\
C2 & 0 & 5 & 33 & 49 \\
C3 & 0 & 3 & 21 & 9 \\
C4 & 0 & 52 & 9 & 0 \\
C5 & 0 & 50 & 0 & 0 \\
\hline
\end{tabular}

Table 7: Server distribution, Server type D

Tables 4-7 show numbers of servers by the cancellation and hit ratios. We observe that Server type A, which has $37 \%$ and $39 \%$ of the cancellation and execution ratios, have its servers mostly in $\mathrm{C} 1 \mathrm{H} 3$, the categories with low cancellation and high execution ratios. Server type B, which has the high cancellation ratio $92 \%$ and the low execution ratio $8 \%$, are mainly distributed in $\mathrm{C} 5 \mathrm{H} 1$. Server type $\mathrm{C}$, which has the relatively high cancellation ratio $44 \%$ and the execution ratio $43 \%$, exist most heavily on $\mathrm{C} 4 \mathrm{H} 2$. Server type $\mathrm{D}$, which has high cancellation ratio $42 \%$ and execution ratios $52 \%$, are distributed mainly in $\mathrm{C} 4 \mathrm{H} 2$.

\section{Analysis of trading and ordering patterns by server type}

In this section, we analyze the shares of the volumes of the executed orders and the changes in volumes of the submitted orders by server type, during the 10 second intervals of the top 10 trading volume stocks for the four different market situations.

Tables 8 and 9 illustrate an example of order books, which are for Mitsubishi UFJ Financial Group, at 9:10:00 a.m. and 9:10:10 a.m. on 8th July 2015. These tables indicate that the stocks were sold off in the short interval, and as a result, the buying orders in the price range from 875.2 to 875.5 were taken and the selling orders in the range from 875.3 to 875.7 were placed instead.

These changes in the order book are due to different types of orders submitted during the period. The originally placed limit buy orders are either canceled, modified to different 
price levels, or taken by market sell orders, while new limit buy orders are placed at the same time. In contrast, there is no limit sell order in the price range at first. As new market sell orders are executed against the existing limit buy orders, new limit sell orders are placed and the limit sell prices are changed to lower levels.

\begin{tabular}{|c|cc|}
\hline 877.0 & 37,600 & 0 \\
876.9 & 20,300 & 0 \\
876.8 & 6,900 & 0 \\
876.7 & 2,200 & 0 \\
876.6 & 5,400 & 0 \\
876.5 & 18,700 & 0 \\
876.4 & 7,500 & 0 \\
876.3 & 6,100 & 0 \\
876.2 & 7,000 & 0 \\
876.1 & 6,000 & 0 \\
876.0 & 3,300 & 0 \\
875.9 & 4,500 & 0 \\
875.8 & 600 & 0 \\
875.7 & 0 & 0 \\
875.6 & 0 & 0 \\
875.5 & 0 & 4,300 \\
875.4 & 0 & 8,400 \\
875.3 & 0 & 3,500 \\
875.2 & 0 & 3,900 \\
875.1 & 0 & 3,300 \\
875.0 & 0 & 20,900 \\
874.9 & 0 & 32,000 \\
874.8 & 0 & 1,900 \\
874.7 & 0 & 5,100 \\
874.6 & 0 & 42,900 \\
874.5 & 0 & 6,500 \\
874.4 & 0 & 9,200 \\
874.3 & 0 & 21,800 \\
874.2 & 0 & 12,200 \\
874.1 & 0 & 400 \\
874.0 & 0 & 34,100 \\
\hline
\end{tabular}

Table 8: Order book at the beginning of the period.

\begin{tabular}{|c|cc|}
\hline 877.0 & 35,400 & 0 \\
876.9 & 20,300 & 0 \\
876.8 & 5,600 & 0 \\
876.7 & 600 & 0 \\
876.6 & 2,500 & 0 \\
876.5 & 13,700 & 0 \\
876.4 & 6,900 & 0 \\
876.3 & 2,600 & 0 \\
876.2 & 9,900 & 0 \\
876.1 & 6,600 & 0 \\
876.0 & 16,000 & 0 \\
875.9 & 5,800 & 0 \\
875.8 & 2,200 & 0 \\
875.7 & 7,000 & 0 \\
875.6 & 4,500 & 0 \\
875.5 & 3,100 & 0 \\
875.4 & 1,600 & 0 \\
875.3 & 700 & 0 \\
875.2 & 0 & 0 \\
875.1 & 0 & 600 \\
875.0 & 0 & 23,200 \\
874.9 & 0 & 13,500 \\
874.8 & 0 & 2,900 \\
874.7 & 0 & 2,800 \\
874.6 & 0 & 5,500 \\
874.5 & 0 & 7,000 \\
874.4 & 0 & 5,100 \\
874.3 & 0 & 43,600 \\
874.2 & 0 & 8,000 \\
874.1 & 0 & 6,900 \\
874.0 & 0 & 35,200 \\
\hline
\end{tabular}

Table 9: Order book at the end of the period.

\subsection{Execution share and change in order volume}

In this subsection, we examine trading and ordering patterns in different degrees of price movements by server type. First, we observe the case of 1st September 2015 when the index declined most in the year by $3.19 \%$. Tables 10-17 show the shares of the executed volume, changes in order volume, and average numbers of price renewal trades for the four server types. These numbers are calculated as follows. Let $w_{\alpha, \beta, \gamma}, v_{\alpha, \beta, \gamma}$, and $u_{\alpha, \beta, \gamma}$ be the share of executed orders, the change in order volume and the number of price renewal, respectively for the server type $\beta$ in the 10 second periods for the market situation $\alpha$ and the order type $\gamma$. Here, $\alpha \in\{$ negative return greater than $10 \mathrm{bps}$, negative return less than 10 bps, positive return less than 10 bps, positive return greater than 10 bps . Since we are interested in the execution and ordering patterns of servers in market moves, we exclude the ten second intervals with zero return, where the start price and the end price are the same, from our analysis. $\beta \in\{\mathrm{A}, \mathrm{B}, \mathrm{C}, \mathrm{D}\} . \gamma \in\{$ buy, sell $\}$. Let $A_{i}$ be the $i$-th 10 second interval of $[0, T]$. Then, the trading period $[0, T]$ is divided into 1,800 disjoint 10 second intervals as follows.

$$
[0, T]=\coprod_{l=1}^{1800} A_{l}
$$

We note that $\coprod_{l=901}^{1260} A_{l}$ corresponds to the lunch break of the market, which is from 11:30 a.m. to 0:30 p.m. JST. We denote by $L_{\alpha}^{(k)}$ the set of $l \in\{1, \ldots, 1800\}$ where the return 
of stock $k$ in $A_{l}$ is $\alpha$. Here, $k=1, \ldots, 10$ represent the top 10 trading volume stocks as of 30th March 2015: 7203 Toyota, 8306 Mitsubishi UFJ Financial Group, 8411 Mizuho Financial Group, 9984 Softbank, 8316 Mitsui Sumitomo Financial Group, 6758 Sony, 2802 Ajinomoto, 6762 TDK, 7267 Honda, and 7270 Subaru. Here, the four digit numbers represent the ticker codes of the stocks.

Let $l_{\alpha}^{(k)}(j), j=1, \ldots, \# L_{\alpha}^{(k)}$ be elements of $L_{\alpha}^{(k)}$. We define $w_{\alpha, \beta, \gamma}, v_{\alpha, \beta, \gamma}$ and $u_{\alpha, \beta, \gamma}$ as follows.

$$
\begin{aligned}
& w_{\alpha, \beta, \gamma}=\frac{1}{\sum_{k=1}^{10} \# L_{\alpha}^{(k)}} \sum_{k=1}^{10} \sum_{j=1}^{\# L_{\alpha}^{(k)}} \frac{\sum_{i=1}^{N^{(k)}} x_{i, 2}^{(k)} 1_{\left\{x_{i, 1}^{(k)}=2, x_{i, 3}^{(k)}=1_{\{\gamma=\mathrm{sell}\}}, x_{i, 7}^{(k)} \in \mathcal{S}_{\beta}, x_{i, 5}^{(k)} \in A_{l_{\alpha}^{(k)}(j)}\right\}}}{\sum_{i=1}^{N^{(k)}} x_{i, 2}^{(k)} 1_{\left\{x_{i, 1}^{(k)}=2, x_{i, 3}^{(k)}=1_{\{\gamma=\mathrm{sell}\}}, x_{i, 5}^{(k)} \in A_{l_{\alpha}^{(k)}(j)}\right\}}} . \\
& v_{\alpha, \beta, \gamma}=\frac{1}{\sum_{k=1}^{10} \# L_{\alpha}^{(k)}} \sum_{k=1}^{10} \sum_{j=1}^{\# L_{\alpha}^{(k)}} \frac{\sum_{s \in \mathcal{S}_{\beta}} c_{s, A_{l_{\alpha}(j)}^{(k), \gamma}}}{\sum_{i=1}^{N^{(k)}} x_{i, 2}^{(k)} 1_{\left\{x_{i, 1}^{(k)}=2, x_{i, 3}^{(k)}=1_{\{\gamma=\mathrm{sell}\}}, x_{i, 5}^{(k)} \in A_{l_{\alpha}^{(k)}(j)}\right\}}} \\
& u_{\alpha, \beta, \gamma}=\frac{1}{\sum_{k=1}^{10} \# L_{\alpha}^{(k)}} \sum_{k=1}^{10} \sum_{j=1}^{\# L_{\alpha}^{(k)}} \sum_{i=1}^{N^{(k)}} 1_{\left\{x_{i, 1}^{(k)}=2, x_{i, 3}^{(k)}=1_{\{\gamma=\mathrm{sell}\}}, x_{i, 7}^{(k)} \in \mathcal{S}_{\beta}, x_{i, 5}^{(k)} \in A_{l_{\alpha}^{(k)}(j)}, x_{i, 4} \neq x_{i, 8}\right\}},
\end{aligned}
$$

where $\mathcal{S}_{\beta}$ is the set of servers with $\beta$ for the server type.

Here, $c_{s, A}^{(k), b u y}$ and $c_{s, A}^{(k), \text { sell }}$ are the changes in the buying and the selling order volume for the time interval $A$ in $B \subset(0, \infty)$, the price range between the last traded prices at the beginning of $A$ and at the end of $A$, for the server $s$, respectively. They are defined as follows.

$$
\begin{aligned}
c_{s, A}^{(k), \text { buy }} & =\sum_{i=1}^{N^{(k)}} x_{i 2}^{(k)} 1_{\left\{x_{i 1}^{(k)}=1,3,4,5, x_{i 2}^{(k)} \in B, x_{i 3}^{(k)}=0, x_{i 5}^{(k)} \in A, x_{i 7}^{(k)}=s\right\}}, \\
c_{s, A}^{(k), \text { sell }} & =\sum_{i=1}^{N^{(k)}} x_{i 2}^{(k)} 1_{\left\{x_{i 1}^{(k)}=1,3,4,5, x_{i 2}^{(k)} \in B, x_{i 3}^{(k)}=1, x_{i 5}^{(k)} \in A, x_{i 7}^{(k)}=s\right\}} .
\end{aligned}
$$

We observe that Server type A has a large share of trading volume in the rapid market movements. In the 10 second intervals where the stock price declines more than $0.10 \%$ in Tables 10 and 11, the share of selling volume is $35 \%$, while the share of buying volume is 14\%. When the stock price rises more than $0.10 \%$ in Tables 16 and 17 , the buying share is $36 \%$, while the selling share is only $13 \%$. Thus, they take the trading strategy where they follow the short-term trend of the stock price movements. Moreover, we find that they place a large amount of selling orders, $118 \%$ of the executed selling orders, when the stock price falls more than $0.10 \%$ in Table 11 , and they place buying orders, which amount to $147 \%$ of the executed orders, when the stock price rises more than $0.10 \%$ in Table 16.

Server type B has a certain level of shares of executed volumes for both buying and selling sides in all the situations. In cases where the stock price moves more than $0.10 \%$, we observe that the buying share is $21 \%$ when the price falls in Table 10, while it is $13 \%$ 
when the price rises in Table 16. Similarly, the selling share is $26 \%$ when the price rises in Table 17, while it is $17 \%$ when the price falls in Table 11. This implies that Server type B trades against the market moves. Next, for the order placement, they submit large selling orders while canceling the buying orders in the falling markets. This indicates that Server type B takes market-making strategies, where they shift the price levels along with the sharp price falls.

Server type $\mathrm{C}$ has considerable shares of trading volumes for both buying and selling sides in all the market situations. They buy $50 \%$ of the executed volume, while selling $37 \%$ of it in the sharp price falls in Tables 10 and 11 . They also have $50 \%$ share of the selling executed orders while having $40 \%$ share of the buying orders in the rapid price rises in Tables 16 and 17. They submit large selling orders in the price falls, while placing a large volume of buying orders in the price rises.

Server type D constantly has around 10\%-14\% shares of the executed volumes in Tables 10-17. The same things hold true for the other dates in our analysis, 4th and 8th of September, 24th and 26th of August, and 8th and 9th of July in Tables 165-220.

\begin{tabular}{|c|ccc|}
\hline & Executed order volume & Change in order volume & Price renewal \\
\hline Server type A & $14 \%$ & $21 \%$ & 0.87 \\
Server type B & $21 \%$ & $-50 \%$ & 0.44 \\
Server type C & $50 \%$ & $22 \%$ & 1.57 \\
Server type D & $14 \%$ & $10 \%$ & 0.31 \\
\hline
\end{tabular}

Table 10: Intervals with a negative return greater than $0.10 \%$, buy orders

\begin{tabular}{|c|ccc|}
\hline & Executed order volume & Change in order volume & Price renewal \\
\hline Server type A & $35 \%$ & $118 \%$ & 2.06 \\
Server type B & $17 \%$ & $124 \%$ & 1.03 \\
Server type C & $37 \%$ & $90 \%$ & 3.04 \\
Server type D & $11 \%$ & $15 \%$ & 0.96 \\
\hline
\end{tabular}

Table 11: Intervals with a negative return greater than $0.10 \%$, sell orders

\begin{tabular}{|l|ccc|}
\hline & Executed order volume & Change in order volume & Price renewal \\
\hline Server type A & $17 \%$ & $13 \%$ & 0.64 \\
Server type B & $15 \%$ & $-14 \%$ & 0.27 \\
Server type C & $54 \%$ & $51 \%$ & 1.55 \\
Server type D & $14 \%$ & $9 \%$ & 0.39 \\
\hline
\end{tabular}

Table 12: Intervals with a negative return less than $0.10 \%$, buy orders

\begin{tabular}{|c|ccc|}
\hline & Executed order volume & Change in order volume & Price renewal \\
\hline Server type A & $32 \%$ & $109 \%$ & 1.25 \\
Server type B & $18 \%$ & $67 \%$ & 0.66 \\
Server type C & $40 \%$ & $82 \%$ & 2.12 \\
Server type D & $11 \%$ & $20 \%$ & 0.61 \\
\hline
\end{tabular}

Table 13: Intervals with a negative return less than $0.10 \%$, sell orders 


\begin{tabular}{|l|ccc|}
\hline & Executed order volume & Change in order volume & Price renewal \\
\hline Server type A & $34 \%$ & $116 \%$ & 1.30 \\
Server type B & $13 \%$ & $50 \%$ & 0.49 \\
Server type C & $40 \%$ & $92 \%$ & 1.90 \\
Server type D & $12 \%$ & $16 \%$ & 0.52 \\
\hline
\end{tabular}

Table 14: Intervals with a positive return less than $0.10 \%$, buy orders

\begin{tabular}{|l|ccc|}
\hline & Executed order volume & Change in order volume & Price renewal \\
\hline Server type A & $17 \%$ & $15 \%$ & 0.55 \\
Server type B & $19 \%$ & $-12 \%$ & 0.29 \\
Server type C & $54 \%$ & $42 \%$ & 1.36 \\
Server type D & $11 \%$ & $5 \%$ & 0.34 \\
\hline
\end{tabular}

Table 15: Intervals with a positive return less than $0.10 \%$, sell orders

\begin{tabular}{|l|ccc|}
\hline & Executed order volume & Change in order volume & Price renewal \\
\hline Server type A & $36 \%$ & $147 \%$ & 2.37 \\
Server type B & $13 \%$ & $105 \%$ & 1.02 \\
Server type C & $40 \%$ & $74 \%$ & 2.61 \\
Server type D & $10 \%$ & $37 \%$ & 0.77 \\
\hline
\end{tabular}

Table 16: Intervals with a positive return greater than $0.10 \%$, buy orders

\begin{tabular}{|l|ccc|}
\hline & Executed order volume & Change in order volume & Price renewal \\
\hline Server type A & $13 \%$ & $19 \%$ & 0.81 \\
Server type B & $26 \%$ & $-72 \%$ & 0.43 \\
Server type C & $50 \%$ & $33 \%$ & 1.54 \\
Server type D & $12 \%$ & $4 \%$ & 0.35 \\
\hline
\end{tabular}

Table 17: Intervals with a positive return greater than $0.10 \%$, sell orders

Remark 1. If we change the definition of Server type A in Section 2.3 as the servers which are not included in Server type $B$ and do not have any buy/sell executed orders in any one second after the opening of the afternoon sessions of 6th, 7th and 8th of July, the summary of the server types is as in Tables 18 \& 19. Note that the servers in Server type A of this classification are more likely to be the proprietary trading servers dealing only in the opening hours. The proprietary trading servers which sometimes use Itayose are excluded from Server type $A$ in this definition. Tables 20-27 show the execution shares and ordering patterns of the four server types on 8th July 2015 with this classification. We still observe that Server type A has a larger share of selling orders in the price falls and buying orders in the price rises.

\begin{tabular}{|c|c|c|c|c|c|c|c|}
\hline & \# of servers & Share & \# of new orders & Share & \# of cancellation orders & Cancellation ratio & Share \\
\hline Server type A & 1,234 & $28 \%$ & $2,007,917$ & $6 \%$ & 682,501 & $34 \%$ & $3 \%$ \\
\hline Server type B & 1,125 & $25 \%$ & $18,204,160$ & $53 \%$ & $16,734,145$ & $92 \%$ & $71 \%$ \\
\hline Server type C & 1,331 & $30 \%$ & $11,705,850$ & $34 \%$ & $5,130,087$ & $44 \%$ & $22 \%$ \\
\hline Server type D & 456 & $10 \%$ & $2,148,566$ & $6 \%$ & 876,808 & $41 \%$ & $4 \%$ \\
\hline
\end{tabular}

Table 18: Number of servers, new orders, and cancellation orders by server type 


\begin{tabular}{|c|ccc|c|}
\hline & \# of executed orders & Execution ratio & Share & Average volume per order in JPY \\
\hline Server type A & 741,412 & $37 \%$ & $9 \%$ & $3,559,876$ \\
Server type B & $1,529,295$ & $8 \%$ & $18 \%$ & $2,581,234$ \\
Server type C & $5,002,736$ & $43 \%$ & $59 \%$ & $1,011,827$ \\
Server type D & $1,128,471$ & $53 \%$ & $13 \%$ & $8,449,489$ \\
\hline
\end{tabular}

Table 19: Number of executed orders by server type

\begin{tabular}{|l|ccc|}
\hline & Executed order volume & Change in order volume & Price renewal \\
\hline Server type A & $11 \%$ & $22 \%$ & 0.74 \\
Server type B & $21 \%$ & $-50 \%$ & 0.44 \\
Server type C & $52 \%$ & $21 \%$ & 1.67 \\
Server type D & $16 \%$ & $10 \%$ & 0.34 \\
\hline
\end{tabular}

Table 20: Intervals with a negative return greater than $0.10 \%$, buy orders

\begin{tabular}{|l|ccc|}
\hline & Executed order volume & Change in order volume & Price renewal \\
\hline Server type A & $27 \%$ & $103 \%$ & 1.60 \\
Server type B & $17 \%$ & $124 \%$ & 1.03 \\
Server type C & $41 \%$ & $97 \%$ & 3.29 \\
Server type D & $14 \%$ & $23 \%$ & 1.17 \\
\hline
\end{tabular}

Table 21: Intervals with a negative return greater than $0.10 \%$, sell orders

\begin{tabular}{|c|ccc|}
\hline & Executed order volume & Change in order volume & Price renewal \\
\hline Server type A & $15 \%$ & $17 \%$ & 0.54 \\
Server type B & $15 \%$ & $-14 \%$ & 0.27 \\
Server type C & $56 \%$ & $46 \%$ & 1.63 \\
Server type D & $15 \%$ & $10 \%$ & 0.42 \\
\hline
\end{tabular}

Table 22: Intervals with a negative return less than $0.10 \%$, buy orders

\begin{tabular}{|c|ccc|}
\hline & Executed order volume & Change in order volume & Price renewal \\
\hline Server type A & $28 \%$ & $102 \%$ & 1.05 \\
Server type B & $18 \%$ & $67 \%$ & 0.66 \\
Server type C & $42 \%$ & $87 \%$ & 2.24 \\
Server type D & $12 \%$ & $22 \%$ & 0.68 \\
\hline
\end{tabular}

Table 23: Intervals with a negative return less than $0.10 \%$, sell orders

\begin{tabular}{|l|ccc|}
\hline & Executed order volume & Change in order volume & Price renewal \\
\hline Server type A & $30 \%$ & $108 \%$ & 1.11 \\
Server type B & $13 \%$ & $50 \%$ & 0.49 \\
Server type C & $43 \%$ & $97 \%$ & 2.04 \\
Server type D & $14 \%$ & $18 \%$ & 0.58 \\
\hline
\end{tabular}

Table 24: Intervals with a positive return less than $0.10 \%$, buy orders 


\begin{tabular}{|c|ccc|}
\hline & Executed order volume & Change in order volume & Price renewal \\
\hline Server type A & $15 \%$ & $14 \%$ & 0.47 \\
Server type B & $19 \%$ & $-12 \%$ & 0.29 \\
Server type C & $55 \%$ & $43 \%$ & 1.42 \\
Server type D & $11 \%$ & $5 \%$ & 0.35 \\
\hline
\end{tabular}

Table 25: Intervals with a positive return less than $0.10 \%$, sell orders

\begin{tabular}{|l|ccc|}
\hline & Executed order volume & Change in order volume & Price renewal \\
\hline Server type A & $29 \%$ & $129 \%$ & 1.90 \\
Server type B & $13 \%$ & $105 \%$ & 1.02 \\
Server type C & $43 \%$ & $83 \%$ & 2.91 \\
Server type D & $14 \%$ & $47 \%$ & 0.95 \\
\hline
\end{tabular}

Table 26: Intervals with a positive return greater than $0.10 \%$, buy orders

\begin{tabular}{|l|ccc|}
\hline & Executed order volume & Change in order volume & Price renewal \\
\hline Server type A & $11 \%$ & $18 \%$ & 0.67 \\
Server type B & $26 \%$ & $-72 \%$ & 0.43 \\
Server type C & $51 \%$ & $34 \%$ & 1.65 \\
Server type D & $12 \%$ & $4 \%$ & 0.39 \\
\hline
\end{tabular}

Table 27: Intervals with a positive return greater than $0.10 \%$, sell orders

\subsection{Shares of executed orders in the swings and roundabouts}

Next, we investigate the shares of execution volumes in the 10 second intervals in different market thinnesses and returns for the four server types. Specifically, we analyze the dates when a large index decrease or increase was observed in the swings and roundabouts of the Nikkei 225 index in July, August, and September in 2015.

\subsubsection{1st - 9th September}

The Nikkei 225 index dropped by $7.50 \%$ in total in the daytime trading hours of 1 st to 8th of September and increased by $5.70 \%$ on 9 th. Among the first six trading dates in the trend of index fall, we choose 1st, 4th, and 8th of September where the index largely dropped by $3.19 \%, 2.84 \%$ and $2.87 \%$ respectively for the analysis. We note that the averages of the daily returns of the 10 representative stocks are as follows:

\begin{tabular}{|l|c|c|}
\hline Trading date & Nikkei 225 index return & 10 stock average return \\
\hline 1st September & $-3.19 \%$ & $-2.90 \%$ \\
2nd September & $1.31 \%$ & $1.66 \%$ \\
3rd September & $-0.96 \%$ & $-1.31 \%$ \\
4th September & $-2.84 \%$ & $-3.09 \%$ \\
7th September & $1.05 \%$ & $1.30 \%$ \\
8th September & $-2.87 \%$ & $-1.92 \%$ \\
9th September & $5.70 \%$ & $3.56 \%$ \\
\hline
\end{tabular}

Table 28: The Nikkei 225 index and 10 representative stock return 
First, we classify the 10 second intervals of the 10 representative stocks by return and market thinness. We label the intervals with a pair of numbers by the level of return and the market thinness as follows.

Return:

1. negative return greater than $0.20 \%$ in 10 seconds,

2. negative return less than $0.20 \%$ in 10 seconds,

3. positive return less than $0.20 \%$ in 10 seconds,

4. positive return greater than $0.20 \%$ in 10 seconds.

Market thinness: the 10 second price change in basis points divided by the executed volume in JPY multiplied by 10 million is

1. less than 1 ,

2. between 1 and 5 ,

3. greater than 5 .

In other words, 1, 2 and 3 for the market thinness imply that the stock price moves less than $1 \mathrm{bp}, 1-5 \mathrm{bps}$, and more than $5 \mathrm{bps}$, respectively, with JPY 10 million in the interval. Table 29 is a histogram which shows the number of the 10 second intervals for the 10 representative stocks in the date by the return and the market thinness. We observe that for all the dates with the price change 1 and 4 , the numbers of times with the thinness 3 are large compared to these with the thinnesses 1 and 2. This means that the sharp price movements are caused by small volumes of trades.

Tables 30-33, 35-38, 40-43, and 45-48 show the shares of the executed orders and the price renewal orders for the four server types by the return and the market thinness. It is observed that on 1st and 8th, the shares of selling volume for Server type A are biased toward the situations with the returns 1 and 2, which indicates that Server type A sells larger volumes in the price falls than in the price rises.

Figures 1-8 are the scattered diagrams displaying the relation between the executed volume in JPY (the horizontal axis) and the return in bps for the 10 second intervals (the vertical axis). The dots and the cross marks correspond to the intervals when the market is opened and at the beginning of the afternoon session, respectively.

On 9th, when the index rebounded significantly, as the high numbers of times for the thinness 2 compared to the thinness 3 and the horizontally long scatter diagram show, the prices did not move much by small volumes of trades. The shares of Server type A are around 20-30 \% in all the situations, and Server type C has constantly large shares higher than $40 \%$ on the date.

- Tables and figure of 1st September 2015 - Down 3.19\% 


\begin{tabular}{|ll|l|}
\hline Return & Thinness & \# of times \\
\hline 1 & 1 & 2 \\
1 & 2 & 19 \\
1 & 3 & 53 \\
\hline 2 & 1 & 1272 \\
2 & 2 & 3009 \\
2 & 3 & 2144 \\
\hline 3 & 1 & 1101 \\
3 & 2 & 2638 \\
3 & 3 & 2146 \\
\hline 4 & 1 & 1 \\
4 & 2 & 19 \\
4 & 3 & 43 \\
\hline
\end{tabular}

Table 29: Number of times for intervals with different levels of market returns and thinnesses

\begin{tabular}{|ll|cccc|}
\hline Return & Thinness & Server type A & Server type B & Server type C & Server type D \\
\hline 1 & 1 & $6 \%$ & $4 \%$ & $17 \%$ & $72 \%$ \\
1 & 2 & $37 \%$ & $18 \%$ & $38 \%$ & $7 \%$ \\
1 & 3 & $34 \%$ & $17 \%$ & $45 \%$ & $5 \%$ \\
\hline 2 & 1 & $32 \%$ & $19 \%$ & $35 \%$ & $14 \%$ \\
2 & 2 & $35 \%$ & $18 \%$ & $38 \%$ & $10 \%$ \\
2 & 3 & $29 \%$ & $16 \%$ & $44 \%$ & $10 \%$ \\
\hline 3 & 1 & $20 \%$ & $17 \%$ & $49 \%$ & $14 \%$ \\
3 & 2 & $17 \%$ & $18 \%$ & $54 \%$ & $11 \%$ \\
3 & 3 & $14 \%$ & $23 \%$ & $55 \%$ & $8 \%$ \\
\hline 4 & 1 & $47 \%$ & $6 \%$ & $42 \%$ & $6 \%$ \\
4 & 2 & $6 \%$ & $20 \%$ & $58 \%$ & $16 \%$ \\
4 & 3 & $9 \%$ & $39 \%$ & $43 \%$ & $9 \%$ \\
\hline
\end{tabular}

Table 30: Shares of selling orders with different levels of market returns and thinnesses

\begin{tabular}{|ll|cccc|}
\hline Return & Thinness & Server type A & Server type B & Server type C & Server type D \\
\hline 1 & 1 & $12 \%$ & $12 \%$ & $54 \%$ & $23 \%$ \\
1 & 2 & $12 \%$ & $22 \%$ & $50 \%$ & $16 \%$ \\
1 & 3 & $10 \%$ & $31 \%$ & $43 \%$ & $16 \%$ \\
\hline 2 & 1 & $20 \%$ & $12 \%$ & $48 \%$ & $20 \%$ \\
2 & 2 & $16 \%$ & $14 \%$ & $54 \%$ & $15 \%$ \\
2 & 3 & $16 \%$ & $19 \%$ & $56 \%$ & $9 \%$ \\
\hline 3 & 1 & $32 \%$ & $13 \%$ & $39 \%$ & $15 \%$ \\
3 & 2 & $37 \%$ & $14 \%$ & $38 \%$ & $12 \%$ \\
3 & 3 & $33 \%$ & $12 \%$ & $44 \%$ & $11 \%$ \\
\hline 4 & 1 & $9 \%$ & $16 \%$ & $6 \%$ & $69 \%$ \\
4 & 2 & $39 \%$ & $15 \%$ & $38 \%$ & $7 \%$ \\
4 & 3 & $36 \%$ & $14 \%$ & $44 \%$ & $7 \%$ \\
\hline
\end{tabular}

Table 31: Shares of buying orders with different levels of market returns and thinnesses 


\begin{tabular}{|ll|cccc|}
\hline Return & Thinness & Server type A & Server type B & Server type C & Server type D \\
\hline 1 & 1 & $4 \%$ & $2 \%$ & $16 \%$ & $77 \%$ \\
1 & 2 & $25 \%$ & $19 \%$ & $44 \%$ & $12 \%$ \\
1 & 3 & $30 \%$ & $12 \%$ & $52 \%$ & $6 \%$ \\
\hline 2 & 1 & $28 \%$ & $17 \%$ & $38 \%$ & $17 \%$ \\
2 & 2 & $31 \%$ & $15 \%$ & $41 \%$ & $12 \%$ \\
2 & 3 & $27 \%$ & $12 \%$ & $46 \%$ & $11 \%$ \\
\hline 3 & 1 & $23 \%$ & $11 \%$ & $48 \%$ & $15 \%$ \\
3 & 2 & $17 \%$ & $9 \%$ & $46 \%$ & $11 \%$ \\
3 & 3 & $10 \%$ & $5 \%$ & $29 \%$ & $5 \%$ \\
\hline 4 & 1 & $88 \%$ & $1 \%$ & $12 \%$ & $0 \%$ \\
4 & 2 & $15 \%$ & $16 \%$ & $48 \%$ & $11 \%$ \\
4 & 3 & $19 \%$ & $9 \%$ & $33 \%$ & $2 \%$ \\
\hline
\end{tabular}

Table 32: Shares of price renewal selling orders with different levels of market returns and thinnesses

\begin{tabular}{|ll|cccc|}
\hline Return & Thinness & Server type A & Server type B & Server type C & Server type D \\
\hline 1 & 1 & $68 \%$ & $11 \%$ & $13 \%$ & $9 \%$ \\
1 & 2 & $34 \%$ & $9 \%$ & $43 \%$ & $4 \%$ \\
1 & 3 & $17 \%$ & $9 \%$ & $21 \%$ & $6 \%$ \\
\hline 2 & 1 & $25 \%$ & $8 \%$ & $49 \%$ & $14 \%$ \\
2 & 2 & $20 \%$ & $7 \%$ & $45 \%$ & $12 \%$ \\
2 & 3 & $10 \%$ & $3 \%$ & $24 \%$ & $6 \%$ \\
\hline 3 & 1 & $31 \%$ & $10 \%$ & $43 \%$ & $15 \%$ \\
3 & 2 & $35 \%$ & $11 \%$ & $40 \%$ & $13 \%$ \\
3 & 3 & $32 \%$ & $8 \%$ & $44 \%$ & $12 \%$ \\
\hline 4 & 1 & $14 \%$ & $5 \%$ & $11 \%$ & $71 \%$ \\
4 & 2 & $32 \%$ & $13 \%$ & $46 \%$ & $9 \%$ \\
4 & 3 & $35 \%$ & $10 \%$ & $43 \%$ & $11 \%$ \\
\hline
\end{tabular}

Table 33: Shares of price renewal buying orders with different levels of market returns and thinnesses

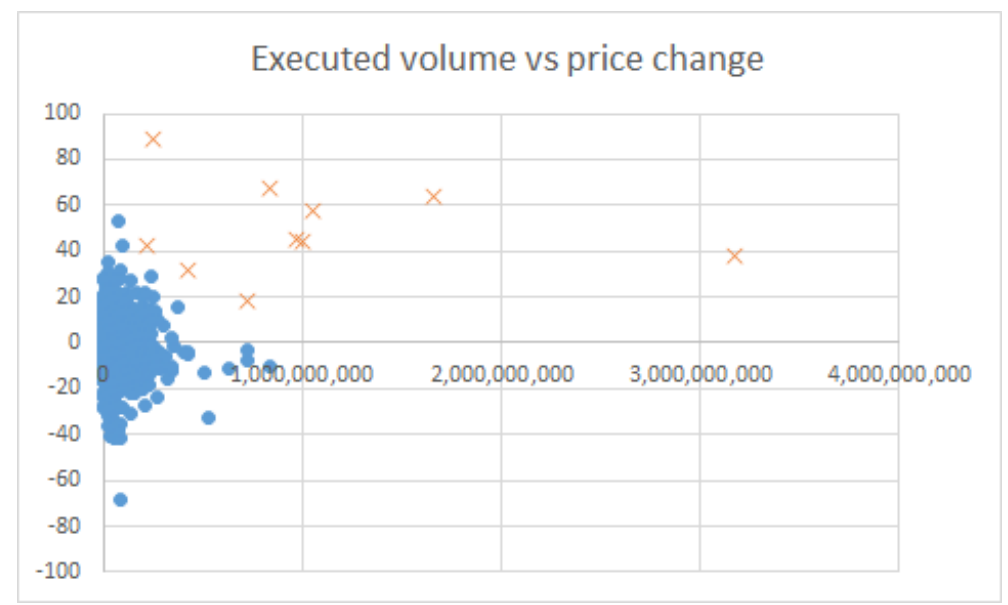

Figure 1: Executed volumes vs price changes, 1st September

- Tables and figure of 4th September 2015 - Down 2.84\% 


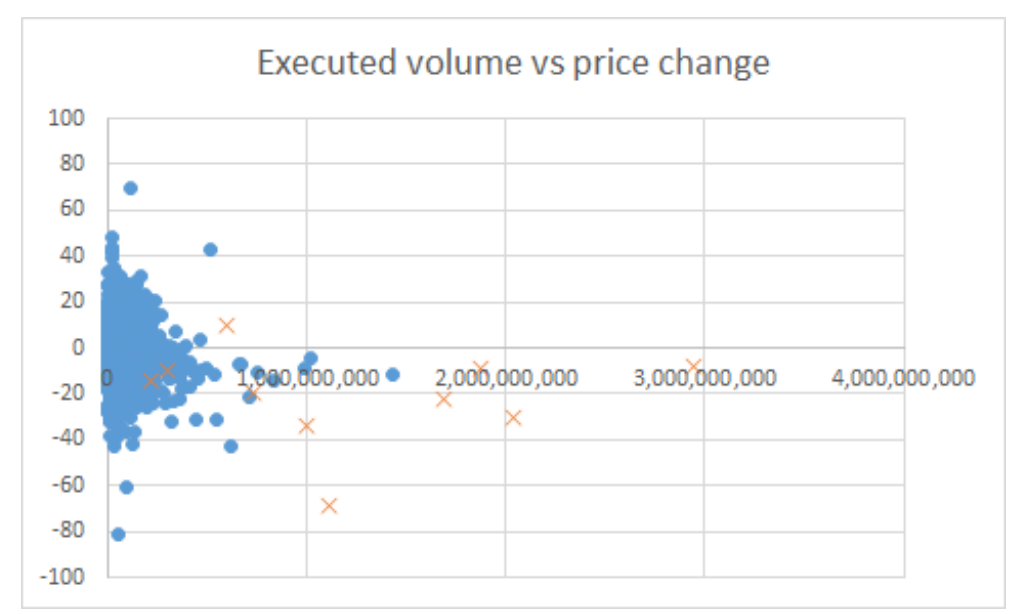

Figure 2: Executed volumes vs price changes, 4th September

\begin{tabular}{|ll|l|}
\hline Return & Thinness & \# of times \\
\hline 1 & 1 & 1 \\
1 & 2 & 17 \\
1 & 3 & 38 \\
\hline 2 & 1 & 828 \\
2 & 2 & 2397 \\
2 & 3 & 2279 \\
\hline 3 & 1 & 815 \\
3 & 2 & 2303 \\
3 & 3 & 2220 \\
\hline 4 & 1 & 2 \\
4 & 2 & 12 \\
4 & 3 & 35 \\
\hline
\end{tabular}

Table 34: Number of times for intervals with different levels of market returns and thinnesses

\begin{tabular}{|ll|cccc|}
\hline Return & Thinness & Server type A & Server type B & Server type C & Server type D \\
\hline 1 & 1 & $60 \%$ & $8 \%$ & $28 \%$ & $5 \%$ \\
1 & 2 & $17 \%$ & $12 \%$ & $55 \%$ & $14 \%$ \\
1 & 3 & $25 \%$ & $31 \%$ & $40 \%$ & $4 \%$ \\
\hline 2 & 1 & $24 \%$ & $18 \%$ & $47 \%$ & $11 \%$ \\
2 & 2 & $25 \%$ & $20 \%$ & $46 \%$ & $10 \%$ \\
2 & 3 & $27 \%$ & $20 \%$ & $44 \%$ & $9 \%$ \\
\hline 3 & 1 & $24 \%$ & $18 \%$ & $46 \%$ & $11 \%$ \\
3 & 2 & $24 \%$ & $18 \%$ & $47 \%$ & $10 \%$ \\
3 & 3 & $26 \%$ & $21 \%$ & $44 \%$ & $9 \%$ \\
\hline 4 & 1 & $3 \%$ & $26 \%$ & $17 \%$ & $53 \%$ \\
4 & 2 & $12 \%$ & $17 \%$ & $50 \%$ & $21 \%$ \\
4 & 3 & $21 \%$ & $21 \%$ & $44 \%$ & $14 \%$ \\
\hline
\end{tabular}

Table 35: Shares of selling orders with different levels of market returns and thinnesses 


\begin{tabular}{|ll|cccc|}
\hline Return & Thinness & Server type A & Server type B & Server type C & Server type D \\
\hline 1 & 1 & $27 \%$ & $72 \%$ & $1 \%$ & $0 \%$ \\
1 & 2 & $29 \%$ & $12 \%$ & $38 \%$ & $21 \%$ \\
1 & 3 & $20 \%$ & $19 \%$ & $55 \%$ & $7 \%$ \\
\hline 2 & 1 & $25 \%$ & $14 \%$ & $49 \%$ & $12 \%$ \\
2 & 2 & $26 \%$ & $15 \%$ & $47 \%$ & $12 \%$ \\
2 & 3 & $26 \%$ & $17 \%$ & $46 \%$ & $10 \%$ \\
\hline 3 & 1 & $25 \%$ & $12 \%$ & $50 \%$ & $12 \%$ \\
3 & 2 & $25 \%$ & $15 \%$ & $48 \%$ & $12 \%$ \\
3 & 3 & $28 \%$ & $16 \%$ & $45 \%$ & $11 \%$ \\
\hline 4 & 1 & $1 \%$ & $3 \%$ & $45 \%$ & $51 \%$ \\
4 & 2 & $22 \%$ & $14 \%$ & $51 \%$ & $12 \%$ \\
4 & 3 & $29 \%$ & $20 \%$ & $41 \%$ & $10 \%$ \\
\hline
\end{tabular}

Table 36: Shares of buying orders with different levels of market returns and thinnesses

\begin{tabular}{|ll|cccc|}
\hline Return & Thinness & Server type A & Server type B & Server type C & Server type D \\
\hline 1 & 1 & $50 \%$ & $0 \%$ & $25 \%$ & $25 \%$ \\
1 & 2 & $27 \%$ & $7 \%$ & $46 \%$ & $15 \%$ \\
1 & 3 & $10 \%$ & $7 \%$ & $34 \%$ & $1 \%$ \\
\hline 2 & 1 & $23 \%$ & $12 \%$ & $49 \%$ & $11 \%$ \\
2 & 2 & $25 \%$ & $12 \%$ & $45 \%$ & $9 \%$ \\
2 & 3 & $26 \%$ & $9 \%$ & $41 \%$ & $9 \%$ \\
\hline 3 & 1 & $23 \%$ & $12 \%$ & $47 \%$ & $11 \%$ \\
3 & 2 & $25 \%$ & $12 \%$ & $45 \%$ & $11 \%$ \\
3 & 3 & $25 \%$ & $11 \%$ & $40 \%$ & $8 \%$ \\
\hline 4 & 1 & $1 \%$ & $0 \%$ & $10 \%$ & $89 \%$ \\
4 & 2 & $9 \%$ & $9 \%$ & $61 \%$ & $20 \%$ \\
4 & 3 & $20 \%$ & $8 \%$ & $32 \%$ & $12 \%$ \\
\hline
\end{tabular}

Table 37: Shares of price renewal selling orders with different levels of market returns and thinnesses

\begin{tabular}{|ll|cccc|}
\hline Return & Thinness & Server type A & Server type B & Server type C & Server type D \\
\hline 1 & 1 & $23 \%$ & $77 \%$ & $0 \%$ & $0 \%$ \\
1 & 2 & $34 \%$ & $13 \%$ & $39 \%$ & $15 \%$ \\
1 & 3 & $14 \%$ & $5 \%$ & $30 \%$ & $7 \%$ \\
\hline 2 & 1 & $26 \%$ & $7 \%$ & $47 \%$ & $11 \%$ \\
2 & 2 & $26 \%$ & $6 \%$ & $47 \%$ & $12 \%$ \\
2 & 3 & $27 \%$ & $6 \%$ & $39 \%$ & $9 \%$ \\
\hline 3 & 1 & $25 \%$ & $6 \%$ & $49 \%$ & $12 \%$ \\
3 & 2 & $26 \%$ & $6 \%$ & $46 \%$ & $12 \%$ \\
3 & 3 & $28 \%$ & $6 \%$ & $38 \%$ & $10 \%$ \\
\hline 4 & 1 & $0 \%$ & $0 \%$ & $33 \%$ & $67 \%$ \\
4 & 2 & $21 \%$ & $4 \%$ & $53 \%$ & $14 \%$ \\
4 & 3 & $19 \%$ & $7 \%$ & $28 \%$ & $5 \%$ \\
\hline
\end{tabular}

Table 38: Shares of price renewal buying orders with different levels of market returns and thinnesses

\section{- Tables and figure of 8th September 2015 - Down $2.87 \%$}




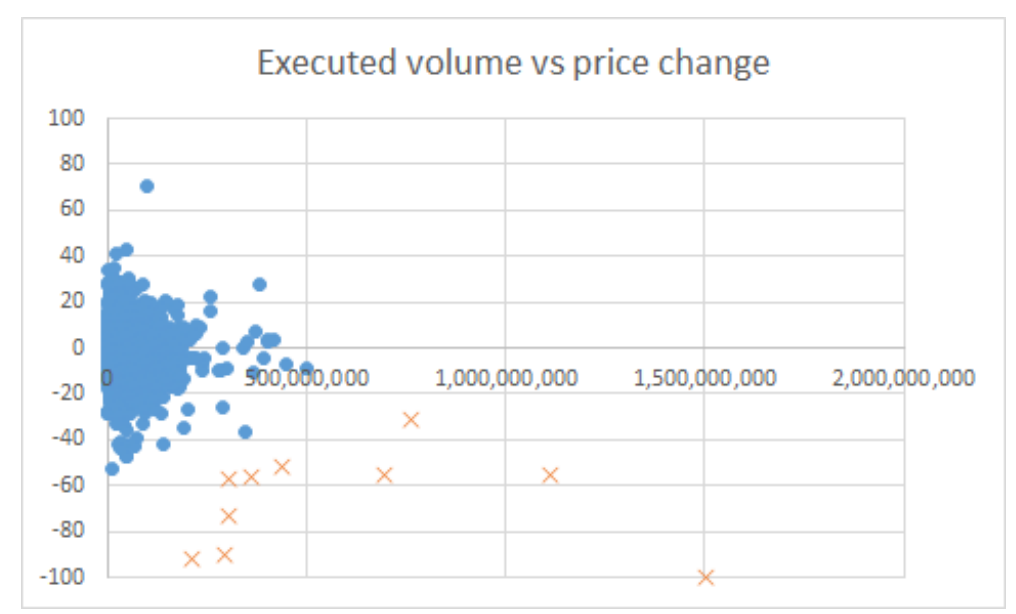

Figure 3: Executed volumes vs price changes, 8th September

\begin{tabular}{|ll|l|}
\hline Return & Thinness & \# of times \\
\hline 1 & 1 & 1 \\
1 & 2 & 20 \\
1 & 3 & 59 \\
\hline 2 & 1 & 835 \\
2 & 2 & 2499 \\
2 & 3 & 2652 \\
\hline 3 & 1 & 826 \\
3 & 2 & 2402 \\
3 & 3 & 2575 \\
\hline 4 & 1 & 2 \\
4 & 2 & 13 \\
4 & 3 & 59 \\
\hline
\end{tabular}

Table 39: Number of times for intervals with different levels of market returns and thinnesses

\begin{tabular}{|ll|cccc|}
\hline Return & Thinness & Server type A & Server type B & Server type C & Server type D \\
\hline 1 & 1 & $58 \%$ & $32 \%$ & $0 \%$ & $10 \%$ \\
1 & 2 & $38 \%$ & $19 \%$ & $36 \%$ & $8 \%$ \\
1 & 3 & $35 \%$ & $16 \%$ & $39 \%$ & $9 \%$ \\
\hline 2 & 1 & $37 \%$ & $16 \%$ & $34 \%$ & $14 \%$ \\
2 & 2 & $38 \%$ & $16 \%$ & $36 \%$ & $10 \%$ \\
2 & 3 & $33 \%$ & $14 \%$ & $45 \%$ & $8 \%$ \\
\hline 3 & 1 & $22 \%$ & $13 \%$ & $47 \%$ & $18 \%$ \\
3 & 2 & $19 \%$ & $17 \%$ & $51 \%$ & $13 \%$ \\
3 & 3 & $15 \%$ & $22 \%$ & $55 \%$ & $8 \%$ \\
\hline 4 & 1 & $6 \%$ & $44 \%$ & $25 \%$ & $25 \%$ \\
4 & 2 & $12 \%$ & $22 \%$ & $46 \%$ & $19 \%$ \\
4 & 3 & $13 \%$ & $37 \%$ & $42 \%$ & $8 \%$ \\
\hline
\end{tabular}

Table 40: Shares of selling orders with different levels of market returns and thinnesses 


\begin{tabular}{|ll|cccc|}
\hline Return & Thinness & Server type A & Server type B & Server type C & Server type D \\
\hline 1 & 1 & $28 \%$ & $25 \%$ & $41 \%$ & $7 \%$ \\
1 & 2 & $10 \%$ & $21 \%$ & $47 \%$ & $22 \%$ \\
1 & 3 & $12 \%$ & $32 \%$ & $47 \%$ & $9 \%$ \\
\hline 2 & 1 & $20 \%$ & $12 \%$ & $52 \%$ & $15 \%$ \\
2 & 2 & $18 \%$ & $16 \%$ & $52 \%$ & $13 \%$ \\
2 & 3 & $18 \%$ & $23 \%$ & $50 \%$ & $8 \%$ \\
\hline 3 & 1 & $33 \%$ & $13 \%$ & $44 \%$ & $11 \%$ \\
3 & 2 & $39 \%$ & $14 \%$ & $38 \%$ & $9 \%$ \\
3 & 3 & $39 \%$ & $13 \%$ & $41 \%$ & $8 \%$ \\
\hline 4 & 1 & $27 \%$ & $4 \%$ & $20 \%$ & $49 \%$ \\
4 & 2 & $36 \%$ & $21 \%$ & $32 \%$ & $10 \%$ \\
4 & 3 & $39 \%$ & $18 \%$ & $36 \%$ & $6 \%$ \\
\hline
\end{tabular}

Table 41: Shares of buying orders with different levels of market returns and thinnesses

\begin{tabular}{|ll|cccc|}
\hline Return & Thinness & Server type A & Server type B & Server type C & Server type D \\
\hline 1 & 1 & $83 \%$ & $17 \%$ & $0 \%$ & $0 \%$ \\
1 & 2 & $39 \%$ & $16 \%$ & $35 \%$ & $9 \%$ \\
1 & 3 & $33 \%$ & $9 \%$ & $49 \%$ & $10 \%$ \\
\hline 2 & 1 & $32 \%$ & $11 \%$ & $43 \%$ & $14 \%$ \\
2 & 2 & $34 \%$ & $11 \%$ & $42 \%$ & $12 \%$ \\
2 & 3 & $30 \%$ & $9 \%$ & $48 \%$ & $9 \%$ \\
\hline 3 & 1 & $23 \%$ & $8 \%$ & $49 \%$ & $13 \%$ \\
3 & 2 & $19 \%$ & $7 \%$ & $47 \%$ & $11 \%$ \\
3 & 3 & $10 \%$ & $5 \%$ & $29 \%$ & $5 \%$ \\
\hline 4 & 1 & $2 \%$ & $38 \%$ & $32 \%$ & $28 \%$ \\
4 & 2 & $28 \%$ & $16 \%$ & $42 \%$ & $14 \%$ \\
4 & 3 & $6 \%$ & $6 \%$ & $38 \%$ & $0 \%$ \\
\hline
\end{tabular}

Table 42: Shares of price renewal selling orders with different levels of market returns and thinnesses

\begin{tabular}{|ll|cccc|}
\hline Return & Thinness & Server type A & Server type B & Server type C & Server type D \\
\hline 1 & 1 & $27 \%$ & $0 \%$ & $73 \%$ & $0 \%$ \\
1 & 2 & $28 \%$ & $17 \%$ & $45 \%$ & $5 \%$ \\
1 & 3 & $12 \%$ & $4 \%$ & $23 \%$ & $0 \%$ \\
\hline 2 & 1 & $25 \%$ & $7 \%$ & $52 \%$ & $11 \%$ \\
2 & 2 & $21 \%$ & $6 \%$ & $46 \%$ & $9 \%$ \\
2 & 3 & $12 \%$ & $3 \%$ & $25 \%$ & $4 \%$ \\
\hline 3 & 1 & $34 \%$ & $10 \%$ & $42 \%$ & $13 \%$ \\
3 & 2 & $38 \%$ & $9 \%$ & $42 \%$ & $10 \%$ \\
3 & 3 & $37 \%$ & $7 \%$ & $43 \%$ & $8 \%$ \\
\hline 4 & 1 & $2 \%$ & $1 \%$ & $48 \%$ & $49 \%$ \\
4 & 2 & $33 \%$ & $12 \%$ & $45 \%$ & $10 \%$ \\
4 & 3 & $33 \%$ & $15 \%$ & $41 \%$ & $11 \%$ \\
\hline
\end{tabular}

Table 43: Shares of price renewal buying orders with different levels of market returns and thinnesses

- Tables and figure of 9th September 2015 - Up 5.70\% 


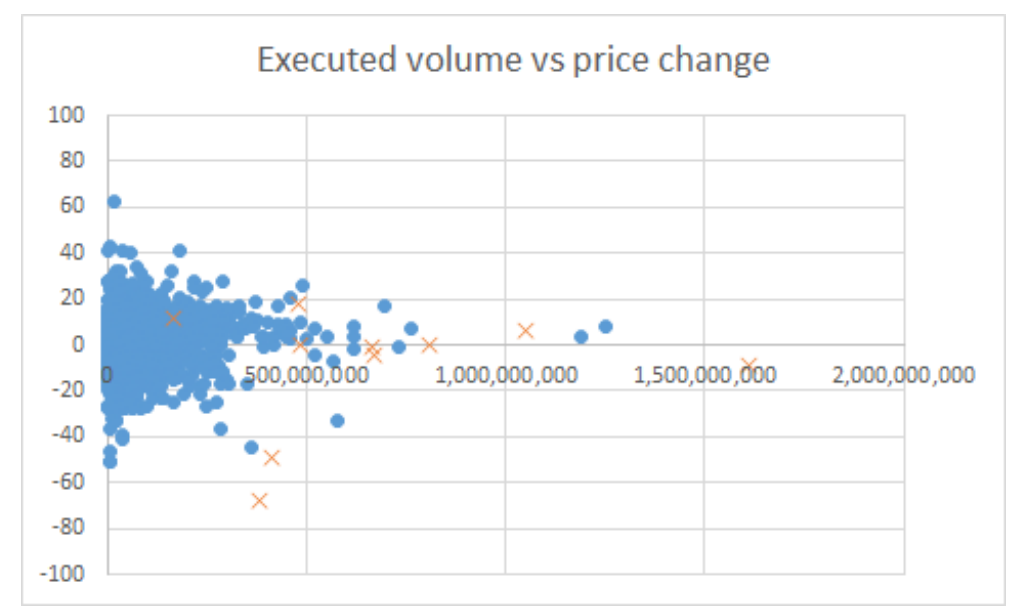

Figure 4: Executed volumes vs price changes, 9th September

\begin{tabular}{|ll|l|}
\hline Return & Thinness & \# of times \\
\hline 1 & 1 & 3 \\
1 & 2 & 18 \\
1 & 3 & 30 \\
\hline 2 & 1 & 1272 \\
2 & 2 & 2550 \\
2 & 3 & 1630 \\
\hline 3 & 1 & 1625 \\
3 & 2 & 2627 \\
3 & 3 & 1675 \\
\hline 4 & 1 & 4 \\
4 & 2 & 27 \\
4 & 3 & 27 \\
\hline
\end{tabular}

Table 44: Number of times for intervals with different levels of market returns and thinnesses

\begin{tabular}{|ll|cccc|}
\hline Return & Thinness & Server type A & Server type B & Server type C & Server type D \\
\hline 1 & 1 & $28 \%$ & $31 \%$ & $40 \%$ & $1 \%$ \\
1 & 2 & $20 \%$ & $16 \%$ & $52 \%$ & $11 \%$ \\
1 & 3 & $16 \%$ & $19 \%$ & $51 \%$ & $14 \%$ \\
\hline 2 & 1 & $22 \%$ & $12 \%$ & $53 \%$ & $12 \%$ \\
2 & 2 & $22 \%$ & $13 \%$ & $53 \%$ & $12 \%$ \\
2 & 3 & $22 \%$ & $15 \%$ & $52 \%$ & $11 \%$ \\
\hline 3 & 1 & $20 \%$ & $12 \%$ & $54 \%$ & $14 \%$ \\
3 & 2 & $20 \%$ & $14 \%$ & $53 \%$ & $12 \%$ \\
3 & 3 & $24 \%$ & $16 \%$ & $50 \%$ & $11 \%$ \\
\hline 4 & 1 & $2 \%$ & $6 \%$ & $68 \%$ & $24 \%$ \\
4 & 2 & $20 \%$ & $21 \%$ & $49 \%$ & $11 \%$ \\
4 & 3 & $18 \%$ & $29 \%$ & $41 \%$ & $12 \%$ \\
\hline
\end{tabular}

Table 45: Shares of selling orders with different levels of market returns and thinnesses 


\begin{tabular}{|ll|cccc|}
\hline Return & Thinness & Server type A & Server type B & Server type C & Server type D \\
\hline 1 & 1 & $26 \%$ & $18 \%$ & $54 \%$ & $1 \%$ \\
1 & 2 & $27 \%$ & $13 \%$ & $50 \%$ & $10 \%$ \\
1 & 3 & $18 \%$ & $24 \%$ & $40 \%$ & $18 \%$ \\
\hline 2 & 1 & $24 \%$ & $12 \%$ & $50 \%$ & $14 \%$ \\
2 & 2 & $25 \%$ & $13 \%$ & $48 \%$ & $13 \%$ \\
2 & 3 & $29 \%$ & $13 \%$ & $47 \%$ & $11 \%$ \\
\hline 3 & 1 & $28 \%$ & $12 \%$ & $48 \%$ & $13 \%$ \\
3 & 2 & $27 \%$ & $13 \%$ & $47 \%$ & $13 \%$ \\
3 & 3 & $28 \%$ & $13 \%$ & $48 \%$ & $11 \%$ \\
\hline 4 & 1 & $32 \%$ & $13 \%$ & $46 \%$ & $9 \%$ \\
4 & 2 & $20 \%$ & $19 \%$ & $47 \%$ & $14 \%$ \\
4 & 3 & $29 \%$ & $18 \%$ & $47 \%$ & $6 \%$ \\
\hline
\end{tabular}

Table 46: Shares of buying orders with different levels of market returns and thinnesses

\begin{tabular}{|ll|cccc|}
\hline Return & Thinness & Server type A & Server type B & Server type C & Server type D \\
\hline 1 & 1 & $33 \%$ & $27 \%$ & $38 \%$ & $2 \%$ \\
1 & 2 & $22 \%$ & $9 \%$ & $44 \%$ & $20 \%$ \\
1 & 3 & $14 \%$ & $3 \%$ & $27 \%$ & $13 \%$ \\
\hline 2 & 1 & $19 \%$ & $6 \%$ & $55 \%$ & $16 \%$ \\
2 & 2 & $19 \%$ & $6 \%$ & $52 \%$ & $13 \%$ \\
2 & 3 & $21 \%$ & $6 \%$ & $46 \%$ & $8 \%$ \\
\hline 3 & 1 & $17 \%$ & $7 \%$ & $55 \%$ & $15 \%$ \\
3 & 2 & $18 \%$ & $6 \%$ & $53 \%$ & $12 \%$ \\
3 & 3 & $21 \%$ & $6 \%$ & $44 \%$ & $10 \%$ \\
\hline 4 & 1 & $7 \%$ & $20 \%$ & $20 \%$ & $28 \%$ \\
4 & 2 & $21 \%$ & $7 \%$ & $51 \%$ & $18 \%$ \\
4 & 3 & $16 \%$ & $1 \%$ & $48 \%$ & $6 \%$ \\
\hline
\end{tabular}

Table 47: Shares of price renewal selling orders with different levels of market returns and thinnesses

\begin{tabular}{|ll|cccc|}
\hline Return & Thinness & Server type A & Server type B & Server type C & Server type D \\
\hline 1 & 1 & $22 \%$ & $4 \%$ & $66 \%$ & $8 \%$ \\
1 & 2 & $32 \%$ & $6 \%$ & $50 \%$ & $6 \%$ \\
1 & 3 & $21 \%$ & $4 \%$ & $33 \%$ & $10 \%$ \\
\hline 2 & 1 & $26 \%$ & $6 \%$ & $47 \%$ & $13 \%$ \\
2 & 2 & $27 \%$ & $6 \%$ & $43 \%$ & $11 \%$ \\
2 & 3 & $30 \%$ & $6 \%$ & $35 \%$ & $9 \%$ \\
\hline 3 & 1 & $30 \%$ & $6 \%$ & $44 \%$ & $12 \%$ \\
3 & 2 & $28 \%$ & $6 \%$ & $43 \%$ & $10 \%$ \\
3 & 3 & $30 \%$ & $6 \%$ & $36 \%$ & $9 \%$ \\
\hline 4 & 1 & $20 \%$ & $15 \%$ & $56 \%$ & $8 \%$ \\
4 & 2 & $25 \%$ & $11 \%$ & $51 \%$ & $13 \%$ \\
4 & 3 & $21 \%$ & $3 \%$ & $45 \%$ & $2 \%$ \\
\hline
\end{tabular}

Table 48: Shares of price renewal buying orders with different levels of market returns and thinnesses

\subsubsection{4th - 26th of August}

The Nikkei 225 index fell by $4.81 \%$ in total in the daytime trading hours of 24 th and 25th. The index increased by $2.70 \%$ on $26 \mathrm{th}$. The returns of the 10 representative stocks are as follows: 


\begin{tabular}{|l|c|c|}
\hline Trading date & Nikkei 225 index return & 10 stock average return \\
\hline 24th August & $-2.80 \%$ & $-3.34 \%$ \\
25th August & $-2.01 \%$ & $0.83 \%$ \\
26th August & $2.70 \%$ & $2.16 \%$ \\
\hline
\end{tabular}

Table 49: The Nikkei 225 index and 10 representative stock return

Among the three dates, we focus on the analysis of 24th when the index dropped by $2.80 \%$, and 26 th when the index increased by $2.70 \%$. We observe that on 24 th, the shares of selling orders for Server type A are skewed to the situations with the price falls. It is notable that on 26th, the numbers of the situations with the return 1 and 4 are particularly high and skewed to the market thinness 3. Moreover, Server type A has large shares of buying orders $45 \%$ in the situation with Return 4 \& Thinness 3 and selling orders $51 \%$ in the situations with Return $1 \&$ Thinness 3 . This implies that there were large price fluctuations mainly caused by small volumes of trades of Server type A on this date.

- Tables and figure of 24th August 2015 - Down 2.80\%

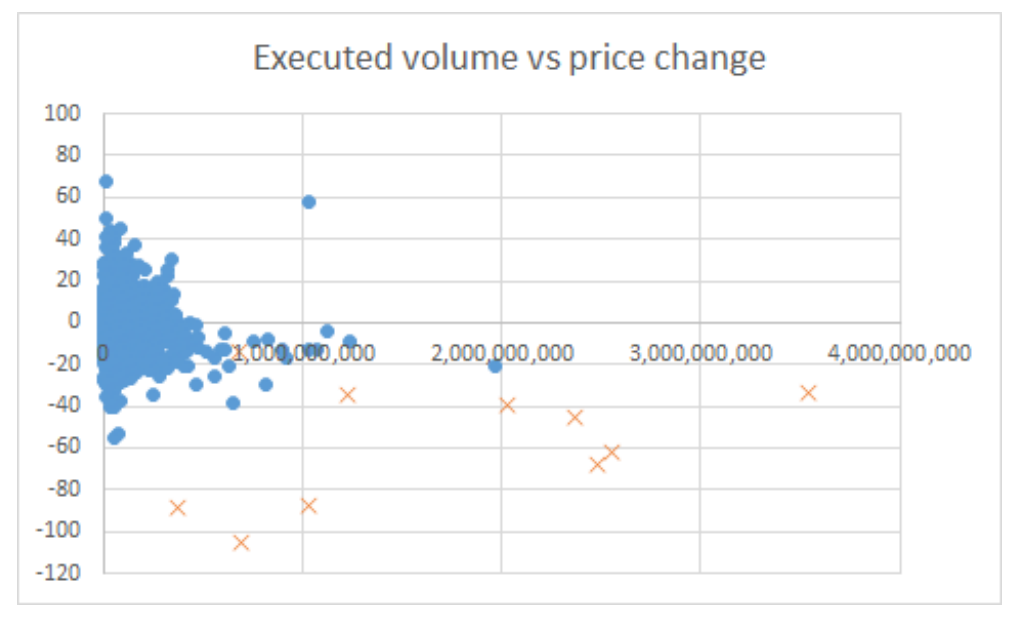

Figure 5: Executed volumes vs price changes, 24th August

\begin{tabular}{|ll|l|}
\hline Return & Thinness & \# of times \\
\hline 1 & 1 & 14 \\
1 & 2 & 25 \\
1 & 3 & 73 \\
\hline 2 & 1 & 2225 \\
2 & 2 & 2894 \\
2 & 3 & 1659 \\
\hline 3 & 1 & 1897 \\
3 & 2 & 2684 \\
3 & 3 & 1553 \\
\hline 4 & 1 & 5 \\
4 & 2 & 39 \\
4 & 3 & 73 \\
\hline
\end{tabular}

Table 50: Number of times for intervals with different levels of market returns and thinnesses 


\begin{tabular}{|ll|cccc|}
\hline Return & Thinness & Server type A & Server type B & Server type C & Server type D \\
\hline 1 & 1 & $13 \%$ & $30 \%$ & $34 \%$ & $16 \%$ \\
1 & 2 & $27 \%$ & $12 \%$ & $45 \%$ & $16 \%$ \\
1 & 3 & $45 \%$ & $10 \%$ & $39 \%$ & $6 \%$ \\
\hline 2 & 1 & $29 \%$ & $13 \%$ & $43 \%$ & $15 \%$ \\
2 & 2 & $32 \%$ & $13 \%$ & $45 \%$ & $10 \%$ \\
2 & 3 & $34 \%$ & $12 \%$ & $46 \%$ & $9 \%$ \\
\hline 3 & 1 & $17 \%$ & $14 \%$ & $51 \%$ & $17 \%$ \\
3 & 2 & $13 \%$ & $17 \%$ & $58 \%$ & $12 \%$ \\
3 & 3 & $11 \%$ & $21 \%$ & $61 \%$ & $8 \%$ \\
\hline 4 & 1 & $8 \%$ & $31 \%$ & $49 \%$ & $11 \%$ \\
4 & 2 & $10 \%$ & $23 \%$ & $55 \%$ & $13 \%$ \\
4 & 3 & $8 \%$ & $28 \%$ & $52 \%$ & $12 \%$ \\
\hline
\end{tabular}

Table 51: Shares of selling orders with different levels of market returns and thinnesses

\begin{tabular}{|ll|cccc|}
\hline Return & Thinness & Server type A & Server type B & Server type C & Server type D \\
\hline 1 & 1 & $14 \%$ & $14 \%$ & $51 \%$ & $21 \%$ \\
1 & 2 & $12 \%$ & $19 \%$ & $48 \%$ & $21 \%$ \\
1 & 3 & $11 \%$ & $43 \%$ & $34 \%$ & $11 \%$ \\
\hline 2 & 1 & $18 \%$ & $11 \%$ & $52 \%$ & $18 \%$ \\
2 & 2 & $16 \%$ & $16 \%$ & $54 \%$ & $15 \%$ \\
2 & 3 & $13 \%$ & $24 \%$ & $53 \%$ & $10 \%$ \\
\hline 3 & 1 & $32 \%$ & $11 \%$ & $41 \%$ & $16 \%$ \\
3 & 2 & $37 \%$ & $11 \%$ & $40 \%$ & $12 \%$ \\
3 & 3 & $36 \%$ & $13 \%$ & $41 \%$ & $10 \%$ \\
\hline 4 & 1 & $13 \%$ & $7 \%$ & $34 \%$ & $46 \%$ \\
4 & 2 & $34 \%$ & $12 \%$ & $40 \%$ & $15 \%$ \\
4 & 3 & $49 \%$ & $13 \%$ & $31 \%$ & $8 \%$ \\
\hline
\end{tabular}

Table 52: Shares of buying orders with different levels of market returns and thinnesses

\begin{tabular}{|ll|cccc|}
\hline Return & Thinness & Server type A & Server type B & Server type C & Server type D \\
\hline 1 & 1 & $10 \%$ & $15 \%$ & $44 \%$ & $24 \%$ \\
1 & 2 & $18 \%$ & $11 \%$ & $53 \%$ & $18 \%$ \\
1 & 3 & $47 \%$ & $7 \%$ & $40 \%$ & $5 \%$ \\
\hline 2 & 1 & $27 \%$ & $9 \%$ & $48 \%$ & $16 \%$ \\
2 & 2 & $31 \%$ & $8 \%$ & $48 \%$ & $12 \%$ \\
2 & 3 & $33 \%$ & $9 \%$ & $45 \%$ & $10 \%$ \\
\hline 3 & 1 & $21 \%$ & $7 \%$ & $55 \%$ & $15 \%$ \\
3 & 2 & $18 \%$ & $6 \%$ & $55 \%$ & $11 \%$ \\
3 & 3 & $10 \%$ & $4 \%$ & $34 \%$ & $5 \%$ \\
\hline 4 & 1 & $12 \%$ & $27 \%$ & $48 \%$ & $14 \%$ \\
4 & 2 & $14 \%$ & $12 \%$ & $57 \%$ & $7 \%$ \\
4 & 3 & $10 \%$ & $7 \%$ & $29 \%$ & $4 \%$ \\
\hline
\end{tabular}

Table 53: Shares of price renewal selling orders with different levels of market returns and thinnesses 


\begin{tabular}{|ll|cccc|}
\hline Return & Thinness & Server type A & Server type B & Server type C & Server type D \\
\hline 1 & 1 & $22 \%$ & $9 \%$ & $48 \%$ & $21 \%$ \\
1 & 2 & $22 \%$ & $11 \%$ & $48 \%$ & $12 \%$ \\
1 & 3 & $14 \%$ & $5 \%$ & $18 \%$ & $4 \%$ \\
\hline 2 & 1 & $24 \%$ & $5 \%$ & $52 \%$ & $16 \%$ \\
2 & 2 & $21 \%$ & $5 \%$ & $52 \%$ & $12 \%$ \\
2 & 3 & $10 \%$ & $3 \%$ & $30 \%$ & $6 \%$ \\
\hline 3 & 1 & $31 \%$ & $6 \%$ & $45 \%$ & $17 \%$ \\
3 & 2 & $36 \%$ & $5 \%$ & $45 \%$ & $13 \%$ \\
3 & 3 & $36 \%$ & $7 \%$ & $43 \%$ & $10 \%$ \\
\hline 4 & 1 & $4 \%$ & $5 \%$ & $31 \%$ & $60 \%$ \\
4 & 2 & $33 \%$ & $7 \%$ & $42 \%$ & $18 \%$ \\
4 & 3 & $48 \%$ & $10 \%$ & $34 \%$ & $7 \%$ \\
\hline
\end{tabular}

Table 54: Shares of price renewal buying orders with different levels of market returns and thinnesses

- Tables and figure of 26th August 2015 - Up 2.70\%

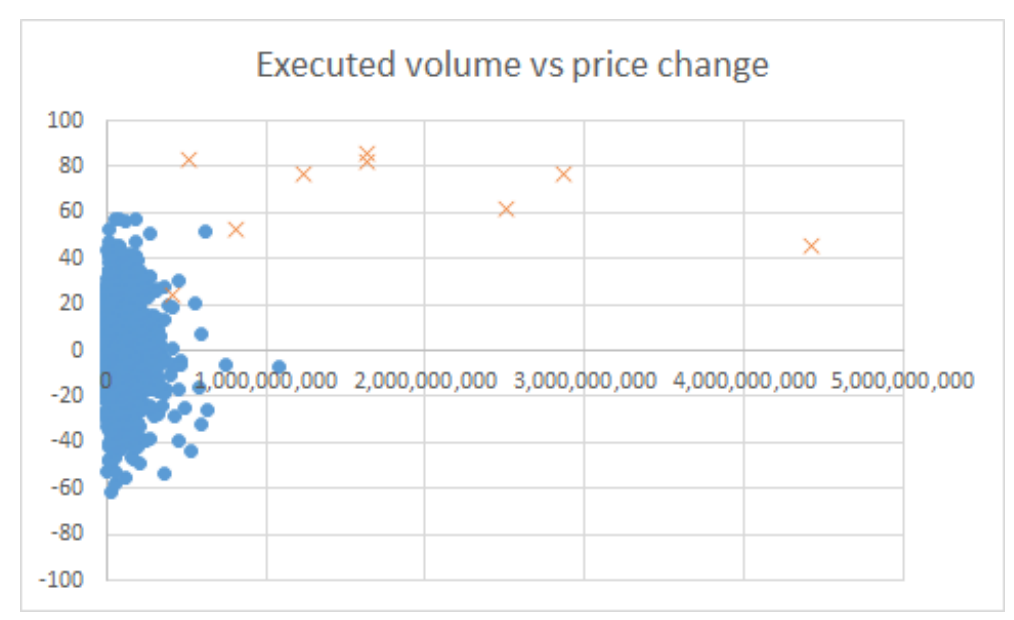

Figure 6: Executed volumes vs price changes, 26th August

\begin{tabular}{|ll|l|}
\hline Return & Thinness & \# of times \\
\hline 1 & 1 & 12 \\
1 & 2 & 131 \\
1 & 3 & 212 \\
\hline 2 & 1 & 1488 \\
2 & 2 & 3018 \\
2 & 3 & 1858 \\
\hline 3 & 1 & 1445 \\
3 & 2 & 2928 \\
3 & 3 & 2303 \\
\hline 4 & 1 & 11 \\
4 & 2 & 144 \\
4 & 3 & 241 \\
\hline
\end{tabular}

Table 55: Number of times for intervals with different levels of market returns and thinnesses 


\begin{tabular}{|ll|cccc|}
\hline Return & Thinness & Server type A & Server type B & Server type C & Server type D \\
\hline 1 & 1 & $26 \%$ & $28 \%$ & $28 \%$ & $17 \%$ \\
1 & 2 & $45 \%$ & $22 \%$ & $22 \%$ & $10 \%$ \\
1 & 3 & $51 \%$ & $19 \%$ & $24 \%$ & $6 \%$ \\
\hline 2 & 1 & $35 \%$ & $16 \%$ & $39 \%$ & $11 \%$ \\
2 & 2 & $39 \%$ & $17 \%$ & $35 \%$ & $9 \%$ \\
2 & 3 & $40 \%$ & $16 \%$ & $37 \%$ & $7 \%$ \\
\hline 3 & 1 & $24 \%$ & $15 \%$ & $47 \%$ & $14 \%$ \\
3 & 2 & $23 \%$ & $17 \%$ & $47 \%$ & $13 \%$ \\
3 & 3 & $19 \%$ & $21 \%$ & $50 \%$ & $10 \%$ \\
\hline 4 & 1 & $11 \%$ & $27 \%$ & $43 \%$ & $20 \%$ \\
4 & 2 & $21 \%$ & $22 \%$ & $43 \%$ & $14 \%$ \\
4 & 3 & $16 \%$ & $30 \%$ & $42 \%$ & $12 \%$ \\
\hline
\end{tabular}

Table 56: Shares of selling orders with different levels of market returns and thinnesses

\begin{tabular}{|ll|cccc|}
\hline Return & Thinness & Server type A & Server type B & Server type C & Server type D \\
\hline 1 & 1 & $12 \%$ & $15 \%$ & $52 \%$ & $20 \%$ \\
1 & 2 & $20 \%$ & $18 \%$ & $48 \%$ & $14 \%$ \\
1 & 3 & $15 \%$ & $28 \%$ & $45 \%$ & $13 \%$ \\
\hline 2 & 1 & $23 \%$ & $13 \%$ & $47 \%$ & $17 \%$ \\
2 & 2 & $20 \%$ & $15 \%$ & $50 \%$ & $14 \%$ \\
2 & 3 & $15 \%$ & $20 \%$ & $53 \%$ & $12 \%$ \\
\hline 3 & 1 & $31 \%$ & $14 \%$ & $39 \%$ & $16 \%$ \\
3 & 2 & $35 \%$ & $15 \%$ & $39 \%$ & $11 \%$ \\
3 & 3 & $37 \%$ & $12 \%$ & $41 \%$ & $10 \%$ \\
\hline 4 & 1 & $27 \%$ & $18 \%$ & $25 \%$ & $31 \%$ \\
4 & 2 & $38 \%$ & $17 \%$ & $31 \%$ & $15 \%$ \\
4 & 3 & $45 \%$ & $14 \%$ & $29 \%$ & $11 \%$ \\
\hline
\end{tabular}

Table 57: Shares of buying orders with different levels of market returns and thinnesses

\begin{tabular}{|ll|cccc|}
\hline Return & Thinness & Server type A & Server type B & Server type C & Server type D \\
\hline 1 & 1 & $22 \%$ & $17 \%$ & $37 \%$ & $25 \%$ \\
1 & 2 & $46 \%$ & $15 \%$ & $25 \%$ & $14 \%$ \\
1 & 3 & $54 \%$ & $13 \%$ & $25 \%$ & $7 \%$ \\
\hline 2 & 1 & $38 \%$ & $11 \%$ & $39 \%$ & $11 \%$ \\
2 & 2 & $41 \%$ & $11 \%$ & $38 \%$ & $9 \%$ \\
2 & 3 & $40 \%$ & $13 \%$ & $38 \%$ & $7 \%$ \\
\hline 3 & 1 & $34 \%$ & $11 \%$ & $43 \%$ & $11 \%$ \\
3 & 2 & $32 \%$ & $9 \%$ & $43 \%$ & $8 \%$ \\
3 & 3 & $20 \%$ & $7 \%$ & $30 \%$ & $5 \%$ \\
\hline 4 & 1 & $35 \%$ & $9 \%$ & $48 \%$ & $8 \%$ \\
4 & 2 & $43 \%$ & $10 \%$ & $35 \%$ & $9 \%$ \\
4 & 3 & $23 \%$ & $9 \%$ & $28 \%$ & $5 \%$ \\
\hline
\end{tabular}

Table 58: Shares of price renewal selling orders with different levels of market returns and thinnesses 


\begin{tabular}{|ll|cccc|}
\hline Return & Thinness & Server type A & Server type B & Server type C & Server type D \\
\hline 1 & 1 & $37 \%$ & $19 \%$ & $33 \%$ & $12 \%$ \\
1 & 2 & $33 \%$ & $8 \%$ & $44 \%$ & $12 \%$ \\
1 & 3 & $25 \%$ & $8 \%$ & $35 \%$ & $7 \%$ \\
\hline 2 & 1 & $28 \%$ & $8 \%$ & $47 \%$ & $17 \%$ \\
2 & 2 & $27 \%$ & $7 \%$ & $46 \%$ & $14 \%$ \\
2 & 3 & $18 \%$ & $5 \%$ & $33 \%$ & $7 \%$ \\
\hline 3 & 1 & $32 \%$ & $8 \%$ & $42 \%$ & $18 \%$ \\
3 & 2 & $36 \%$ & $8 \%$ & $43 \%$ & $13 \%$ \\
3 & 3 & $38 \%$ & $7 \%$ & $41 \%$ & $11 \%$ \\
\hline 4 & 1 & $22 \%$ & $8 \%$ & $35 \%$ & $35 \%$ \\
4 & 2 & $38 \%$ & $10 \%$ & $34 \%$ & $18 \%$ \\
4 & 3 & $46 \%$ & $8 \%$ & $32 \%$ & $13 \%$ \\
\hline
\end{tabular}

Table 59: Shares of price renewal buying orders with different levels of market returns and thinnesses

\subsubsection{8th and 9th July}

- Tables and figure of 8th July 2015 - Down $2.69 \%$

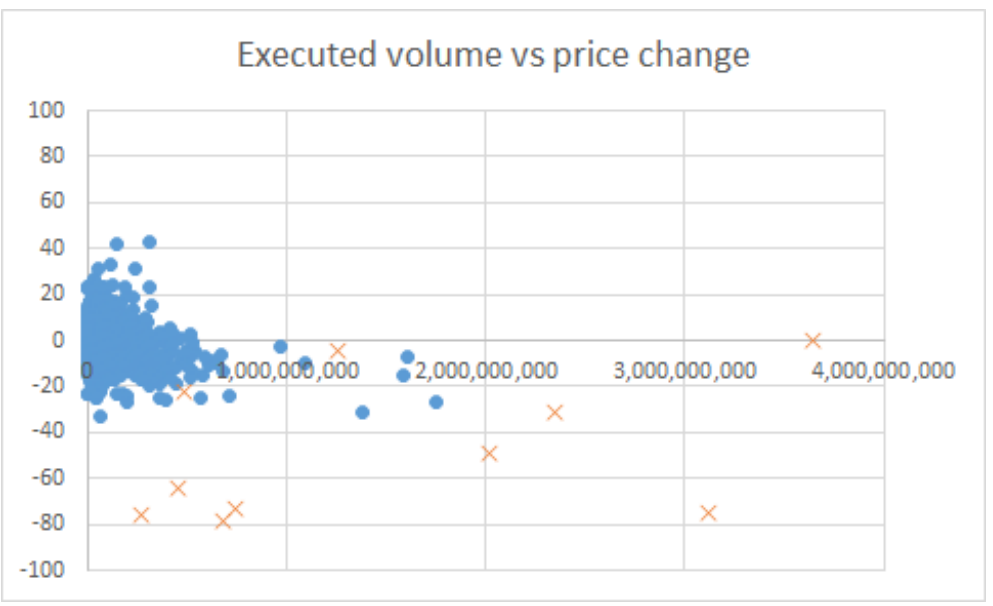

Figure 7: Executed volumes vs price changes, 8th July

The Nikkei 225 index dropped by $2.69 \%$ on 8 th and increased by $2.23 \%$ on 9 th July. The returns of the 10 representative stocks are as follows:

\begin{tabular}{|l|c|c|}
\hline Trading date & Nikkei 225 index return & 10 stock average return \\
\hline 8th July & $-2.69 \%$ & $-2.88 \%$ \\
9th July & $2.23 \%$ & $2.73 \%$ \\
\hline
\end{tabular}

Table 60: The Nikkei 225 index and 10 representative stock return

We observe that on 8th, the shares of the selling volumes for Server type A are skewed to the situations of the price falls and on 9th, the shares of the buying volumes for Server type A are biased toward the situations with the price rises. 


\begin{tabular}{|ll|l|}
\hline Return & Thinness & \# of times \\
\hline 1 & 1 & 6 \\
1 & 2 & 6 \\
1 & 3 & 24 \\
\hline 2 & 1 & 1783 \\
2 & 2 & 2594 \\
2 & 3 & 1696 \\
\hline 3 & 1 & 1511 \\
3 & 2 & 2525 \\
3 & 3 & 1632 \\
\hline 4 & 1 & 1 \\
4 & 2 & 13 \\
4 & 3 & 20 \\
\hline
\end{tabular}

Table 61: Number of times for intervals with different levels of market returns and thinnesses

\begin{tabular}{|ll|cccc|}
\hline Return & Thinness & Server type A & Server type B & Server type C & Server type D \\
\hline 1 & 1 & $13 \%$ & $26 \%$ & $41 \%$ & $21 \%$ \\
1 & 2 & $24 \%$ & $23 \%$ & $30 \%$ & $22 \%$ \\
1 & 3 & $43 \%$ & $9 \%$ & $32 \%$ & $16 \%$ \\
\hline 2 & 1 & $30 \%$ & $15 \%$ & $39 \%$ & $16 \%$ \\
2 & 2 & $33 \%$ & $13 \%$ & $40 \%$ & $13 \%$ \\
2 & 3 & $27 \%$ & $11 \%$ & $50 \%$ & $12 \%$ \\
\hline 3 & 1 & $14 \%$ & $17 \%$ & $53 \%$ & $17 \%$ \\
3 & 2 & $12 \%$ & $18 \%$ & $57 \%$ & $13 \%$ \\
3 & 3 & $13 \%$ & $24 \%$ & $53 \%$ & $10 \%$ \\
\hline 4 & 1 & $9 \%$ & $27 \%$ & $56 \%$ & $8 \%$ \\
4 & 2 & $15 \%$ & $27 \%$ & $49 \%$ & $9 \%$ \\
4 & 3 & $9 \%$ & $32 \%$ & $43 \%$ & $16 \%$ \\
\hline
\end{tabular}

Table 62: Shares of selling orders with different levels of market returns and thinnesses

\begin{tabular}{|ll|cccc|}
\hline Return & Thinness & Server type A & Server type B & Server type C & Server type D \\
\hline 1 & 1 & $8 \%$ & $7 \%$ & $61 \%$ & $22 \%$ \\
1 & 2 & $4 \%$ & $11 \%$ & $58 \%$ & $26 \%$ \\
1 & 3 & $5 \%$ & $19 \%$ & $68 \%$ & $8 \%$ \\
\hline 2 & 1 & $17 \%$ & $12 \%$ & $51 \%$ & $20 \%$ \\
2 & 2 & $14 \%$ & $15 \%$ & $54 \%$ & $16 \%$ \\
2 & 3 & $15 \%$ & $18 \%$ & $56 \%$ & $12 \%$ \\
\hline 3 & 1 & $36 \%$ & $11 \%$ & $37 \%$ & $16 \%$ \\
3 & 2 & $39 \%$ & $10 \%$ & $37 \%$ & $14 \%$ \\
3 & 3 & $31 \%$ & $8 \%$ & $49 \%$ & $12 \%$ \\
\hline 4 & 1 & $17 \%$ & $10 \%$ & $55 \%$ & $18 \%$ \\
4 & 2 & $33 \%$ & $3 \%$ & $41 \%$ & $22 \%$ \\
4 & 3 & $44 \%$ & $8 \%$ & $42 \%$ & $6 \%$ \\
\hline
\end{tabular}

Table 63: Shares of buying orders with different levels of market returns and thinnesses 


\begin{tabular}{|ll|cccc|}
\hline Return & Thinness & Server type A & Server type B & Server type C & Server type D \\
\hline 1 & 1 & $5 \%$ & $11 \%$ & $54 \%$ & $30 \%$ \\
1 & 2 & $21 \%$ & $19 \%$ & $35 \%$ & $25 \%$ \\
1 & 3 & $30 \%$ & $5 \%$ & $40 \%$ & $25 \%$ \\
\hline 2 & 1 & $27 \%$ & $10 \%$ & $45 \%$ & $18 \%$ \\
2 & 2 & $30 \%$ & $9 \%$ & $45 \%$ & $14 \%$ \\
2 & 3 & $25 \%$ & $7 \%$ & $53 \%$ & $11 \%$ \\
\hline 3 & 1 & $16 \%$ & $7 \%$ & $55 \%$ & $16 \%$ \\
3 & 2 & $12 \%$ & $6 \%$ & $47 \%$ & $14 \%$ \\
3 & 3 & $7 \%$ & $3 \%$ & $26 \%$ & $5 \%$ \\
\hline 4 & 1 & $12 \%$ & $7 \%$ & $61 \%$ & $20 \%$ \\
4 & 2 & $7 \%$ & $2 \%$ & $48 \%$ & $5 \%$ \\
4 & 3 & $6 \%$ & $0 \%$ & $19 \%$ & $0 \%$ \\
\hline
\end{tabular}

Table 64: Shares of price renewal selling orders with different levels of market returns and thinnesses

\begin{tabular}{|ll|cccc|}
\hline Return & Thinness & Server type A & Server type B & Server type C & Server type D \\
\hline 1 & 1 & $15 \%$ & $19 \%$ & $42 \%$ & $25 \%$ \\
1 & 2 & $19 \%$ & $1 \%$ & $67 \%$ & $13 \%$ \\
1 & 3 & $4 \%$ & $0 \%$ & $21 \%$ & $0 \%$ \\
\hline 2 & 1 & $19 \%$ & $6 \%$ & $52 \%$ & $18 \%$ \\
2 & 2 & $14 \%$ & $4 \%$ & $48 \%$ & $14 \%$ \\
2 & 3 & $8 \%$ & $2 \%$ & $26 \%$ & $6 \%$ \\
\hline 3 & 1 & $34 \%$ & $8 \%$ & $40 \%$ & $18 \%$ \\
3 & 2 & $35 \%$ & $6 \%$ & $42 \%$ & $16 \%$ \\
3 & 3 & $29 \%$ & $4 \%$ & $52 \%$ & $12 \%$ \\
\hline 4 & 1 & $14 \%$ & $1 \%$ & $79 \%$ & $6 \%$ \\
4 & 2 & $26 \%$ & $1 \%$ & $45 \%$ & $27 \%$ \\
4 & 3 & $41 \%$ & $6 \%$ & $46 \%$ & $7 \%$ \\
\hline
\end{tabular}

Table 65: Shares of price renewal buying orders with different levels of market returns and thinnesses

- Tables and figure of 9th July 2015 - Up $2.23 \%$

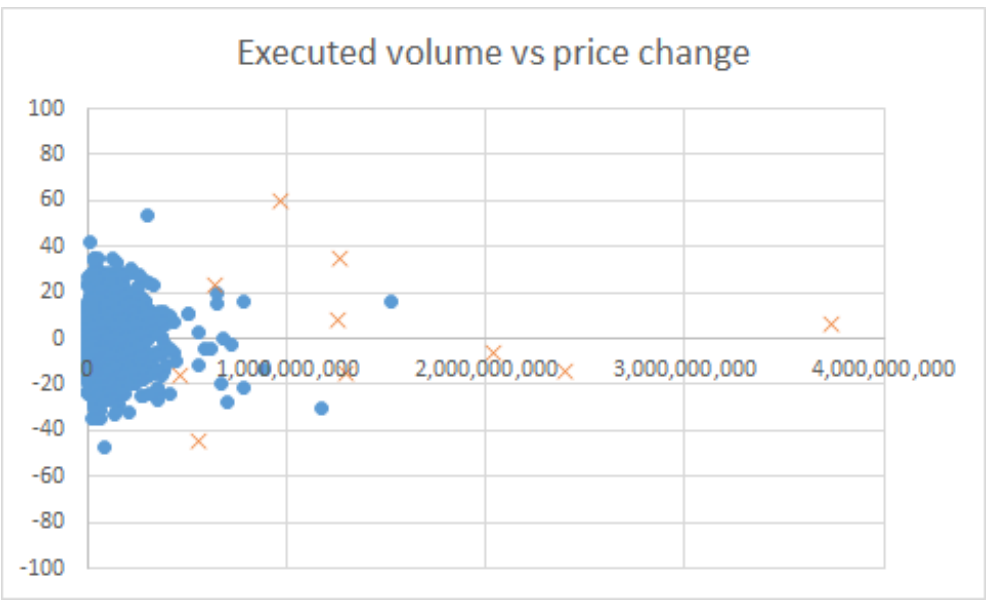

Figure 8: Executed volumes vs price changes, 9th July 


\begin{tabular}{|ll|l|}
\hline Return & Thinness & \# of times \\
\hline 1 & 1 & 10 \\
1 & 2 & 36 \\
1 & 3 & 57 \\
\hline 2 & 1 & 1526 \\
2 & 2 & 2962 \\
2 & 3 & 1716 \\
\hline 3 & 1 & 1620 \\
3 & 2 & 3105 \\
3 & 3 & 2011 \\
\hline 4 & 1 & 9 \\
4 & 2 & 68 \\
4 & 3 & 59 \\
\hline
\end{tabular}

Table 66: Number of times for intervals with different levels of market returns and thinnesses

\begin{tabular}{|ll|cccc|}
\hline Return & Thinness & Server type A & Server type B & Server type C & Server type D \\
\hline 1 & 1 & $19 \%$ & $12 \%$ & $43 \%$ & $26 \%$ \\
1 & 2 & $36 \%$ & $15 \%$ & $30 \%$ & $19 \%$ \\
1 & 3 & $40 \%$ & $12 \%$ & $30 \%$ & $18 \%$ \\
\hline 2 & 1 & $33 \%$ & $15 \%$ & $37 \%$ & $16 \%$ \\
2 & 2 & $39 \%$ & $13 \%$ & $35 \%$ & $14 \%$ \\
2 & 3 & $36 \%$ & $10 \%$ & $43 \%$ & $11 \%$ \\
\hline 3 & 1 & $18 \%$ & $15 \%$ & $48 \%$ & $19 \%$ \\
3 & 2 & $14 \%$ & $17 \%$ & $53 \%$ & $16 \%$ \\
3 & 3 & $13 \%$ & $21 \%$ & $56 \%$ & $10 \%$ \\
\hline 4 & 1 & $16 \%$ & $29 \%$ & $44 \%$ & $11 \%$ \\
4 & 2 & $12 \%$ & $23 \%$ & $42 \%$ & $22 \%$ \\
4 & 3 & $11 \%$ & $28 \%$ & $51 \%$ & $10 \%$ \\
\hline
\end{tabular}

Table 67: Shares of selling orders with different levels of market returns and thinnesses

\begin{tabular}{|ll|cccc|}
\hline Return & Thinness & Server type A & Server type B & Server type C & Server type D \\
\hline 1 & 1 & $24 \%$ & $20 \%$ & $43 \%$ & $14 \%$ \\
1 & 2 & $17 \%$ & $22 \%$ & $36 \%$ & $25 \%$ \\
1 & 3 & $10 \%$ & $38 \%$ & $39 \%$ & $13 \%$ \\
\hline 2 & 1 & $19 \%$ & $13 \%$ & $46 \%$ & $22 \%$ \\
2 & 2 & $15 \%$ & $16 \%$ & $51 \%$ & $18 \%$ \\
2 & 3 & $14 \%$ & $20 \%$ & $53 \%$ & $13 \%$ \\
\hline 3 & 1 & $32 \%$ & $12 \%$ & $36 \%$ & $20 \%$ \\
3 & 2 & $39 \%$ & $11 \%$ & $35 \%$ & $14 \%$ \\
3 & 3 & $34 \%$ & $10 \%$ & $43 \%$ & $13 \%$ \\
\hline 4 & 1 & $21 \%$ & $12 \%$ & $40 \%$ & $27 \%$ \\
4 & 2 & $38 \%$ & $12 \%$ & $30 \%$ & $20 \%$ \\
4 & 3 & $58 \%$ & $9 \%$ & $25 \%$ & $7 \%$ \\
\hline
\end{tabular}

Table 68: Shares of buying orders with different levels of market returns and thinnesses 


\begin{tabular}{|ll|cccc|}
\hline Return & Thinness & Server type A & Server type B & Server type C & Server type D \\
\hline 1 & 1 & $9 \%$ & $4 \%$ & $47 \%$ & $29 \%$ \\
1 & 2 & $32 \%$ & $6 \%$ & $38 \%$ & $24 \%$ \\
1 & 3 & $37 \%$ & $6 \%$ & $37 \%$ & $18 \%$ \\
\hline 2 & 1 & $30 \%$ & $9 \%$ & $42 \%$ & $18 \%$ \\
2 & 2 & $36 \%$ & $7 \%$ & $40 \%$ & $16 \%$ \\
2 & 3 & $35 \%$ & $6 \%$ & $45 \%$ & $11 \%$ \\
\hline 3 & 1 & $20 \%$ & $8 \%$ & $49 \%$ & $18 \%$ \\
3 & 2 & $15 \%$ & $7 \%$ & $47 \%$ & $15 \%$ \\
3 & 3 & $8 \%$ & $3 \%$ & $28 \%$ & $5 \%$ \\
\hline 4 & 1 & $25 \%$ & $9 \%$ & $50 \%$ & $16 \%$ \\
4 & 2 & $15 \%$ & $7 \%$ & $46 \%$ & $26 \%$ \\
4 & 3 & $12 \%$ & $1 \%$ & $23 \%$ & $2 \%$ \\
\hline
\end{tabular}

Table 69: Shares of price renewal selling orders with different levels of market returns and thinnesses

\begin{tabular}{|ll|cccc|}
\hline Return & Thinness & Server type A & Server type B & Server type C & Server type D \\
\hline 1 & 1 & $36 \%$ & $13 \%$ & $36 \%$ & $15 \%$ \\
1 & 2 & $18 \%$ & $7 \%$ & $46 \%$ & $22 \%$ \\
1 & 3 & $15 \%$ & $2 \%$ & $20 \%$ & $8 \%$ \\
\hline 2 & 1 & $20 \%$ & $5 \%$ & $51 \%$ & $22 \%$ \\
2 & 2 & $16 \%$ & $5 \%$ & $51 \%$ & $17 \%$ \\
2 & 3 & $9 \%$ & $2 \%$ & $34 \%$ & $7 \%$ \\
\hline 3 & 1 & $29 \%$ & $7 \%$ & $42 \%$ & $22 \%$ \\
3 & 2 & $36 \%$ & $5 \%$ & $42 \%$ & $16 \%$ \\
3 & 3 & $32 \%$ & $6 \%$ & $46 \%$ & $13 \%$ \\
\hline 4 & 1 & $17 \%$ & $3 \%$ & $48 \%$ & $32 \%$ \\
4 & 2 & $30 \%$ & $8 \%$ & $40 \%$ & $22 \%$ \\
4 & 3 & $55 \%$ & $11 \%$ & $27 \%$ & $7 \%$ \\
\hline
\end{tabular}

Table 70: Shares of price renewal buying orders with different levels of market returns and thinnesses

\subsection{Shares of executed orders on a quiet day: Tables and figure of 17 th July 2015 - Up $0.02 \%$}

In this subsection, we present shares of buying/selling for the four server types on 17th July 2015 as an example of a low volatility day as opposed to the days in the swings and roundabouts in Section 3.2. 


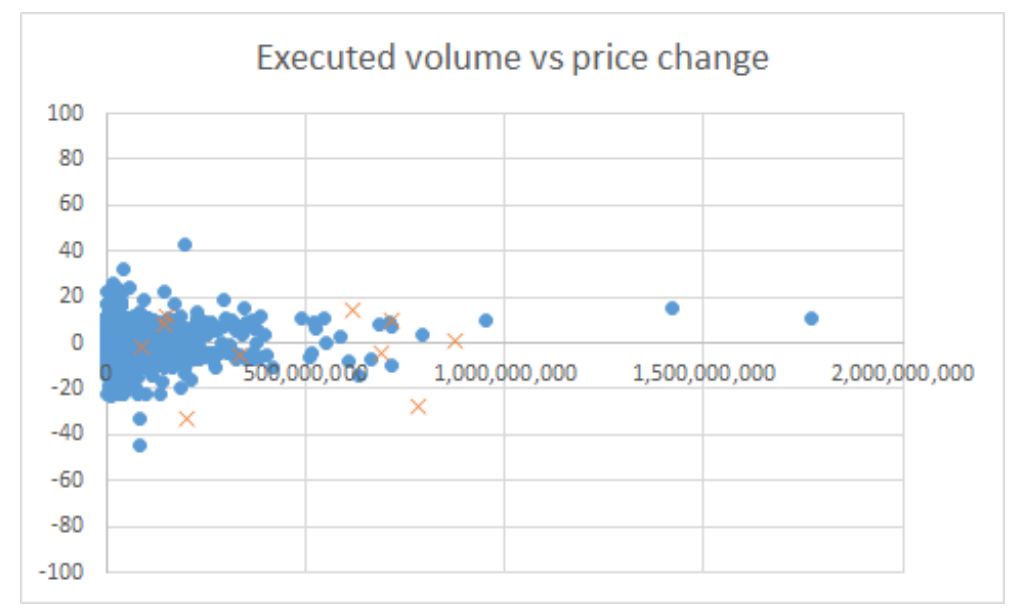

Figure 9: Executed volumes vs price changes, 17th July

The horizontally long scattered diagram indicates that the market was thick. The Nikkei 225 index is almost unchanged on 17 th; it increased only by $0.02 \%$. The returns of the 10 representative stocks are as follows:

\begin{tabular}{|l|c|c|}
\hline Trading date & Nikkei 225 index return & 10 stock average return \\
\hline 8th July & $+0.02 \%$ & $+0.22 \%$ \\
\hline
\end{tabular}

Table 71: The Nikkei 225 index and 10 representative stock return

Although most of the price movements are small in the day, we observe similar trading patterns to the days in the swings and roundabouts for each server type. Server type A takes a trend following strategy; we observe that the shares of the selling/buying volumes for Server type A are skewed to the situations of the price falls/increases. Server types B \& D have constant shares in both buying and selling, and Server type C has large shares.

\begin{tabular}{|ll|l|}
\hline Return & Thinness & \# of times \\
\hline 1 & 1 & 0 \\
1 & 2 & 3 \\
1 & 3 & 7 \\
\hline 2 & 1 & 1041 \\
2 & 2 & 1411 \\
2 & 3 & 1196 \\
\hline 3 & 1 & 1031 \\
3 & 2 & 1414 \\
3 & 3 & 1236 \\
\hline 4 & 1 & 0 \\
4 & 2 & 0 \\
4 & 3 & 7 \\
\hline
\end{tabular}

Table 72: Number of times for intervals with different levels of market returns and thinnesses 


\begin{tabular}{|ll|cccc|}
\hline Return & Thinness & Server type A & Server type B & Server type C & Server type D \\
\hline 1 & 1 & N/A & N/A & N/A & N/A \\
1 & 2 & $30 \%$ & $14 \%$ & $25 \%$ & $9 \%$ \\
1 & 3 & $25 \%$ & $10 \%$ & $48 \%$ & $14 \%$ \\
\hline 2 & 1 & $21 \%$ & $12 \%$ & $48 \%$ & $19 \%$ \\
2 & 2 & $21 \%$ & $10 \%$ & $52 \%$ & $17 \%$ \\
2 & 3 & $15 \%$ & $9 \%$ & $59 \%$ & $18 \%$ \\
\hline 3 & 1 & $8 \%$ & $11 \%$ & $61 \%$ & $19 \%$ \\
3 & 2 & $10 \%$ & $13 \%$ & $60 \%$ & $17 \%$ \\
3 & 3 & $14 \%$ & $16 \%$ & $54 \%$ & $16 \%$ \\
\hline 4 & 1 & $\mathrm{~N} / \mathrm{A}$ & $\mathrm{N} / \mathrm{A}$ & $\mathrm{N} / \mathrm{A}$ & $\mathrm{N} / \mathrm{A}$ \\
4 & 2 & $\mathrm{~N} / \mathrm{A}$ & $\mathrm{N} / \mathrm{A}$ & $\mathrm{N} / \mathrm{A}$ & $\mathrm{N} / \mathrm{A}$ \\
4 & 3 & $13 \%$ & $16 \%$ & $67 \%$ & $5 \%$ \\
\hline
\end{tabular}

Table 73: Shares of selling orders with different levels of market returns and thinnesses

\begin{tabular}{|ll|cccc|}
\hline Return & Thinness & Server type A & Server type B & Server type C & Server type D \\
\hline 1 & 1 & N/A & N/A & N/A & N/A \\
1 & 2 & $47 \%$ & $12 \%$ & $26 \%$ & $14 \%$ \\
1 & 3 & $8 \%$ & $21 \%$ & $65 \%$ & $6 \%$ \\
\hline 2 & 1 & $9 \%$ & $14 \%$ & $57 \%$ & $19 \%$ \\
2 & 2 & $10 \%$ & $14 \%$ & $61 \%$ & $14 \%$ \\
2 & 3 & $13 \%$ & $15 \%$ & $61 \%$ & $11 \%$ \\
\hline 3 & 1 & $24 \%$ & $15 \%$ & $43 \%$ & $19 \%$ \\
3 & 2 & $24 \%$ & $12 \%$ & $49 \%$ & $15 \%$ \\
3 & 3 & $17 \%$ & $10 \%$ & $60 \%$ & $12 \%$ \\
\hline 4 & 1 & N/A & N/A & N/A & N/A \\
4 & 2 & N/A & N/A & N/A & N \\
4 & 3 & $39 \%$ & $14 \%$ & $36 \%$ & $11 \%$ \\
\hline
\end{tabular}

Table 74: Shares of buying orders with different levels of market returns and thinnesses

\begin{tabular}{|ll|cccc|}
\hline Return & Thinness & Server type A & Server type B & Server type C & Server type D \\
\hline 1 & 1 & N/A & N/A & N/A & N/A \\
1 & 2 & $53 \%$ & $4 \%$ & $38 \%$ & $1 \%$ \\
1 & 3 & $10 \%$ & $2 \%$ & $55 \%$ & $22 \%$ \\
\hline 2 & 1 & $15 \%$ & $10 \%$ & $54 \%$ & $21 \%$ \\
2 & 2 & $15 \%$ & $7 \%$ & $58 \%$ & $18 \%$ \\
2 & 3 & $12 \%$ & $6 \%$ & $60 \%$ & $16 \%$ \\
\hline 3 & 1 & $7 \%$ & $6 \%$ & $56 \%$ & $14 \%$ \\
3 & 2 & $6 \%$ & $3 \%$ & $45 \%$ & $9 \%$ \\
3 & 3 & $3 \%$ & $2 \%$ & $21 \%$ & $5 \%$ \\
\hline 4 & 1 & N/A & N/A & N/A & N/A \\
4 & 2 & N/A & N/A & N/A & N/A \\
4 & 3 & $0 \%$ & $0 \%$ & $0 \%$ & $0 \%$ \\
\hline
\end{tabular}

Table 75: Shares of price renewal selling orders with different levels of market returns and thinnesses 


\begin{tabular}{|ll|cccc|}
\hline Return & Thinness & Server type A & Server type B & Server type C & Server type D \\
\hline 1 & 1 & N/A & N/A & N/A & N/A \\
1 & 2 & $31 \%$ & $9 \%$ & $26 \%$ & $0 \%$ \\
1 & 3 & $14 \%$ & $14 \%$ & $43 \%$ & $0 \%$ \\
\hline 2 & 1 & $8 \%$ & $7 \%$ & $57 \%$ & $14 \%$ \\
2 & 2 & $7 \%$ & $4 \%$ & $40 \%$ & $9 \%$ \\
2 & 3 & $4 \%$ & $2 \%$ & $18 \%$ & $3 \%$ \\
\hline 3 & 1 & $18 \%$ & $10 \%$ & $51 \%$ & $21 \%$ \\
3 & 2 & $20 \%$ & $10 \%$ & $53 \%$ & $15 \%$ \\
3 & 3 & $15 \%$ & $8 \%$ & $61 \%$ & $11 \%$ \\
\hline 4 & 1 & N/A & N/A & N/A & N/A \\
4 & 2 & N/A & N/A & N/A & N/A \\
4 & 3 & $34 \%$ & $25 \%$ & $25 \%$ & $16 \%$ \\
\hline
\end{tabular}

Table 76: Shares of price renewal buying orders with different levels of market returns and thinnesses

\section{Buying levels in the price falls and P\&L of Server type B}

As we have observed in Section 3.1, Server type B has certain shares of buying orders in the price falling situations. In order to examine trading patterns of the server type in the situations in more detail, this section investigates the price levels where Server type B buys in the price falls. Particularly, we further classify the servers in Server type B by aggressiveness and observe their shares and execution levels in the price falls. In addition, we observe P\&L of Server type B on trading of the 10 stocks on two price falling days, 8th July and 1st September 2015.

\subsection{Buying levels of Server type B in the price falls}

Firstly, we investigate at which levels the servers bought the stocks in the price falling intervals with a negative return more than 10 bps or 20 bps on 8th July and 1st September 2015. For each server type, we take the volume weighted average of the buying levels over the price falling intervals. The levels are expressed in percentage where $100 \%$ is the high price and $0 \%$ is the low price in the 10 seconds. The servers are classified based on the trading data of all the listed stocks in TSE of the corresponding date. Aggressiveness of a server is defined as the ratio of aggressively executed orders to all orders executed by the server. We say that an order is aggressively executed when the server buys or sells the stock by taking orders placed in the opposite side of the order book, which is identified in our data set.

Tables $77 \& 78$ show the buying levels of Server type B and the other server types on 8th July 2015 in the intervals with negative returns more than 10 bps and 20 bps, respectively. Tables 79 \& 80 illustrate the buying levels of servers in Server type B further classified by the aggressiveness. In these tables, the shares are calculated as the ratio of the executed volume of the server type to that of the total servers.

We observe that in these price falling situations, Server type B buys at lower levels than the other server types. Moreover, the servers with aggressiveness 0-20\% in Server type B has the largest share. The same observation holds for 1st September 2015 as in Tables $81-84$. 


\begin{tabular}{|l|c|c|}
\hline & Share & Buying level \\
\hline Server type B & $21 \%$ & $20 \%$ \\
others & $79 \%$ & $33 \%$ \\
\hline
\end{tabular}

Table 77: Server type B's buying level in the intervals with negative return greater than 10 bps, 8th July 2015

\begin{tabular}{|l|c|c|}
\hline & Share & Buying level \\
\hline Server type B & $16 \%$ & $21 \%$ \\
others & $84 \%$ & $38 \%$ \\
\hline
\end{tabular}

Table 78: Server type B's buying level in the intervals with negative return greater than 20 bps, 8th July 2015

\begin{tabular}{|l|c|c|}
\hline Server type B & Share & Buying level \\
\hline Aggressiveness 0.8-1.0 & $0 \%$ & N/A \\
Aggressiveness 0.6-0.8 & $0 \%$ & $26 \%$ \\
Aggressiveness 0.4-0.6 & $2 \%$ & $21 \%$ \\
Aggressiveness 0.2-0.4 & $3 \%$ & $28 \%$ \\
Aggressiveness 0.0-0.2 & $16 \%$ & $18 \%$ \\
\hline
\end{tabular}

Table 79: Server type B's buying level by aggressiveness in the intervals with negative return greater than 10 bps, 8th July 2015

\begin{tabular}{|l|c|c|}
\hline Server type B & Share & Buying level \\
\hline Aggressiveness 0.8-1.0 & $0 \%$ & N/A \\
Aggressiveness 0.6-0.8 & $0 \%$ & $56 \%$ \\
Aggressiveness 0.4-0.6 & $1 \%$ & $34 \%$ \\
Aggressiveness 0.2-0.4 & $2 \%$ & $41 \%$ \\
Aggressiveness 0.0-0.2 & $13 \%$ & $17 \%$ \\
\hline
\end{tabular}

Table 80: Server type B's buying level by aggressiveness in the intervals with negative return greater than 20 bps, 8th July 2015

\begin{tabular}{|l|c|c|}
\hline & Share & Buying level \\
\hline Server type B & $24 \%$ & $28 \%$ \\
others & $76 \%$ & $36 \%$ \\
\hline
\end{tabular}

Table 81: Server type B's buying level in the intervals with negative return greater than 10 bps, 1st September 2015

\begin{tabular}{|l|c|c|}
\hline & Share & Buying level \\
\hline Server type B & $31 \%$ & $35 \%$ \\
Others & $69 \%$ & $42 \%$ \\
\hline
\end{tabular}

Table 82: Server type B's buying level in the intervals with negative return greater than 20 bps, 1st September 2015 


\begin{tabular}{|l|c|c|}
\hline Server type B & Share & Buying level \\
\hline Aggressiveness 0.8-1.0 & $0 \%$ & $51 \%$ \\
Aggressiveness 0.6-0.8 & $0 \%$ & $51 \%$ \\
Aggressiveness 0.4-0.6 & $1 \%$ & $41 \%$ \\
Aggressiveness 0.2-0.4 & $5 \%$ & $37 \%$ \\
Aggressiveness 0.0-0.2 & $16 \%$ & $24 \%$ \\
\hline
\end{tabular}

Table 83: Server type B's buying level by aggressiveness in the intervals with negative return greater than 10 bps, 1st September 2015

\begin{tabular}{|l|c|c|}
\hline Server type B & Share & Buying level \\
\hline Aggressiveness 0.8-1.0 & $0 \%$ & $42 \%$ \\
Aggressiveness 0.6-0.8 & $0 \%$ & $37 \%$ \\
Aggressiveness 0.4-0.6 & $1 \%$ & $49 \%$ \\
Aggressiveness 0.2-0.4 & $7 \%$ & $47 \%$ \\
Aggressiveness 0.0-0.2 & $22 \%$ & $30 \%$ \\
\hline
\end{tabular}

Table 84: Server type B's buying level by aggressiveness in the intervals with negative return greater than 20 bps, 1st September 2015

\subsection{Execution share and change in order volume of Server type B by aggressiveness}

Tables 85-92 show the execution shares and changes in order volume of Server type B by aggressiveness on 1st September 2015. This corresponds to Server type B in Tables 10-17 in Section 3.1. Here, the classification is based on the order data on 8th July 2015 as in Section 3.1. We observe that cancellation of buying orders in the price falls is done by passive servers with aggressiveness $0.0-0.2$, and the addition of the selling orders in the price falls are done by the servers with aggressiveness $0.2-0.4$. We also observe that the selling/buying execution shares in the price falls/rises are mostly had by passive servers with aggressiveness $0.0-0.2$.

\begin{tabular}{|c|ccc|}
\hline & Executed order volume & Change in order volume & Price renewal \\
\hline Aggressiveness 0.8-1.0 & $0 \%$ & $0 \%$ & 0.00 \\
Aggressiveness 0.6-0.8 & $0 \%$ & $-4 \%$ & 0.02 \\
Aggressiveness 0.4-0.6 & $1 \%$ & $11 \%$ & 0.08 \\
Aggressiveness 0.2-0.4 & $2 \%$ & $37 \%$ & 0.13 \\
Aggressiveness 0.0-0.2 & $18 \%$ & $-88 \%$ & 0.21 \\
\hline
\end{tabular}

Table 85: Intervals with a negative return greater than $0.10 \%$, buy orders

\begin{tabular}{|c|ccc|}
\hline & Executed order volume & Change in order volume & Price renewal \\
\hline Aggressiveness 0.8-1.0 & $0 \%$ & $0 \%$ & 0.00 \\
Aggressiveness 0.6-0.8 & $1 \%$ & $-1 \%$ & 0.09 \\
Aggressiveness 0.4-0.6 & $5 \%$ & $24 \%$ & 0.36 \\
Aggressiveness 0.2-0.4 & $4 \%$ & $114 \%$ & 0.22 \\
Aggressiveness 0.0-0.2 & $7 \%$ & $-25 \%$ & 0.36 \\
\hline
\end{tabular}

Table 86: Intervals with a negative return greater than $0.10 \%$, sell orders 


\begin{tabular}{|c|ccc|}
\hline & Executed order volume & Change in order volume & Price renewal \\
\hline Aggressiveness 0.8-1.0 & $0 \%$ & $0 \%$ & 0.00 \\
Aggressiveness 0.6-0.8 & $0 \%$ & $0 \%$ & 0.03 \\
Aggressiveness 0.4-0.6 & $1 \%$ & $12 \%$ & 0.06 \\
Aggressiveness 0.2-0.4 & $2 \%$ & $54 \%$ & 0.08 \\
Aggressiveness 0.0-0.2 & $10 \%$ & $-51 \%$ & 0.10 \\
\hline
\end{tabular}

Table 87: Intervals with a negative return less than $0.10 \%$, buy orders

\begin{tabular}{|c|ccc|}
\hline & Executed order volume & Change in order volume & Price renewal \\
\hline Aggressiveness 0.8-1.0 & $0 \%$ & $0 \%$ & 0.00 \\
Aggressiveness 0.6-0.8 & $1 \%$ & $0 \%$ & 0.03 \\
Aggressiveness 0.4-0.6 & $6 \%$ & $21 \%$ & 0.28 \\
Aggressiveness 0.2-0.4 & $4 \%$ & $90 \%$ & 0.16 \\
Aggressiveness 0.0-0.2 & $7 \%$ & $-22 \%$ & 0.19 \\
\hline
\end{tabular}

Table 88: Intervals with a negative return less than $0.10 \%$, sell orders

\begin{tabular}{|c|ccc|}
\hline & Executed order volume & Change in order volume & Price renewal \\
\hline Aggressiveness 0.8-1.0 & $0 \%$ & $0 \%$ & 0.00 \\
Aggressiveness 0.6-0.8 & $1 \%$ & $0 \%$ & 0.04 \\
Aggressiveness 0.4-0.6 & $3 \%$ & $36 \%$ & 0.15 \\
Aggressiveness 0.2-0.4 & $3 \%$ & $102 \%$ & 0.12 \\
Aggressiveness 0.0-0.2 & $6 \%$ & $-26 \%$ & 0.18 \\
\hline
\end{tabular}

Table 89: Intervals with a positive return less than $0.10 \%$, buy orders

\begin{tabular}{|c|ccc|}
\hline & Executed order volume & Change in order volume & Price renewal \\
\hline Aggressiveness 0.8-1.0 & $0 \%$ & $0 \%$ & 0.00 \\
Aggressiveness 0.6-0.8 & $1 \%$ & $0 \%$ & 0.02 \\
Aggressiveness 0.4-0.6 & $3 \%$ & $-1 \%$ & 0.10 \\
Aggressiveness 0.2-0.4 & $3 \%$ & $52 \%$ & 0.06 \\
Aggressiveness 0.0-0.2 & $13 \%$ & $-50 \%$ & 0.10 \\
\hline
\end{tabular}

Table 90: Intervals with a positive return less than $0.10 \%$, sell orders

\begin{tabular}{|c|ccc|}
\hline & Executed order volume & Change in order volume & Price renewal \\
\hline Aggressiveness 0.8-1.0 & $0 \%$ & $0 \%$ & 0.00 \\
Aggressiveness 0.6-0.8 & $1 \%$ & $0 \%$ & 0.06 \\
Aggressiveness 0.4-0.6 & $2 \%$ & $52 \%$ & 0.16 \\
Aggressiveness 0.2-0.4 & $3 \%$ & $102 \%$ & 0.33 \\
Aggressiveness 0.0-0.2 & $8 \%$ & $-34 \%$ & 0.49 \\
\hline
\end{tabular}

Table 91: Intervals with a positive return greater than $0.10 \%$, buy orders

\begin{tabular}{|c|ccc|}
\hline & Executed order volume & Change in order volume & Price renewal \\
\hline Aggressiveness 0.8-1.0 & $0 \%$ & $0 \%$ & 0.00 \\
Aggressiveness 0.6-0.8 & $0 \%$ & $-5 \%$ & 0.04 \\
Aggressiveness 0.4-0.6 & $1 \%$ & $2 \%$ & 0.12 \\
Aggressiveness 0.2-0.4 & $3 \%$ & $28 \%$ & 0.09 \\
Aggressiveness 0.0-0.2 & $21 \%$ & $-92 \%$ & 0.18 \\
\hline
\end{tabular}

Table 92: Intervals with a positive return greater than $0.10 \%$, sell orders 


\subsection{P\&L of Server type B}

Next, we investigate P\&L that Server type B made on 8th July and 1st of September 2015 in trading of the 10 stocks. Table 93 shows P\&L of Server type B and the other server types together with their returns on trading volume for the 10 stocks on 8th July 2015. The trading volume is calculated as the net cash amount paid/received on buying/selling the stocks. Table 94 presents those of servers in Server type B further classified by the aggressiveness. P\&L of a server is defined as the mark-to-market of its position at the end of the day. Tables $95 \& 96$ are the same analysis for 1st September 2015. Sections 4.2.1 and 4.2.2 present the corresponding results by ticker.

We observe that on both 8th July and 1st September 2015 when the stock prices fell largely, Server type B made a profit on trading of the stocks from the other server types. In detail, Server type B earned a profit on 10 out of 10 stocks on 8th July and 9 out of 10 stocks on 1st September 2015. Particularly, Server type B with aggressiveness 40 - 60\% made a large portion of Server type B' profit with high returns on trading volume. Server type B with aggressiveness $0-20 \%$ has the largest share in trading volume and shows a constant profit.

\begin{tabular}{|l|c|cc|c|}
\hline & Share & P\&L & Total volume in cash & P\&L vs Total volume \\
\hline Server type B & $14 \%$ & $336,540,010$ & $134,772,260,696$ & $0.25 \%$ \\
Others & $86 \%$ & $-335,466,010$ & $827,602,148,834$ & $-0.04 \%$ \\
\hline
\end{tabular}

Table 93: P\&L of Server type B and the others, 8th July 2015

\begin{tabular}{|l|c|cc|c|}
\hline Server type B & Share & P\&L & Total volume in cash & P\&L vs Total volume \\
\hline Aggressiveness 0.8-1.0 & $0 \%$ & 0 & 0 & N/A \\
Aggressiveness 0.6-0.8 & $1 \%$ & $18,746,210$ & $4,880,967,365$ & $0.38 \%$ \\
Aggressiveness 0.4-0.6 & $3 \%$ & $196,431,190$ & $24,746,065,522$ & $0.79 \%$ \\
Aggressiveness 0.2-0.4 & $3 \%$ & $38,632,820$ & $31,944,427,532$ & $0.12 \%$ \\
Aggressiveness 0.0-0.2 & $8 \%$ & $82,734,430$ & $75,683,095,172$ & $0.11 \%$ \\
\hline
\end{tabular}

Table 94: P\&L of Server type B by aggressiveness, 8th July 2015

\begin{tabular}{|l|c|cc|c|}
\hline & Share & P\&L & Total volume in cash & P\&L vs total volume \\
\hline Server type B & $22 \%$ & $207,084,590$ & $164,108,079,984$ & $0.13 \%$ \\
others & $78 \%$ & $-207,084,590$ & $588,853,030,054$ & $-0.04 \%$ \\
\hline
\end{tabular}

Table 95: P\&L of Server type B and the others, 1st September 2015

\begin{tabular}{|l|c|cc|c|}
\hline Server type B & Share & P\&L & Total volume in cash & P\&L vs total volume \\
\hline Aggressiveness 0.8-1.0 & $1 \%$ & $9,553,330$ & $11,194,668,720$ & $0.09 \%$ \\
Aggressiveness 0.6-0.8 & $2 \%$ & $27,046,050$ & $11,595,633,917$ & $0.23 \%$ \\
Aggressiveness 0.4-0.6 & $4 \%$ & $94,644,080$ & $28,753,740,717$ & $0.33 \%$ \\
Aggressiveness 0.2-0.4 & $6 \%$ & $-18,010,500$ & $43,568,305,655$ & $-0.04 \%$ \\
Aggressiveness 0.0-0.2 & $9 \%$ & $93,940,410$ & $68,479,613,585$ & $0.14 \%$ \\
\hline
\end{tabular}

Table 96: P\&L of Server type B by aggressiveness, 1st September 2015 


\subsubsection{P\&L of Server type B by ticker, 8th July 2015}

Table 97 shows daily returns of the 10 stocks on 8th July 2015.

\begin{tabular}{|l|cc|c|}
\hline Ticker & Start price & End price & Daily return \\
\hline 8316 & 5461 & 5230 & $-4.23 \%$ \\
8411 & 264 & 253.3 & $-4.05 \%$ \\
8306 & 875 & 840.5 & $-3.94 \%$ \\
6758 & 3445 & 3322 & $-3.57 \%$ \\
6762 & 8990 & 8720 & $-3.00 \%$ \\
7270 & 4428.5 & 4318.5 & $-2.48 \%$ \\
7267 & 3930 & 3835 & $-2.42 \%$ \\
9984 & 7084 & 6934 & $-2.12 \%$ \\
7203 & 8092 & 7931 & $-1.99 \%$ \\
2802 & 2628.5 & 2602 & $-1.01 \%$ \\
\hline
\end{tabular}

Table 97: 8th July 2015 Daily return by ticker

Tables 98-117 show P\&L of Server type B corresponding to Tables 93 \& 94 for each individual stock.

- 8316

\begin{tabular}{|l|c|cc|c|}
\hline & Share & P\&L & Total volume in cash & P\&L vs total volume \\
\hline Server type B & $16 \%$ & $41,105,300$ & $20,579,500,040$ & $0.20 \%$ \\
others & $84 \%$ & $-41,105,300$ & $107,999,925,528$ & $-0.04 \%$ \\
\hline
\end{tabular}

Table 98: 8th July 2015 P\&L, 8316

\begin{tabular}{|l|c|cc|c|}
\hline Server type B & Share & P\&L & Total volume in cash & P\&L vs total volume \\
\hline Aggressiveness 0.8-1.0 & $0 \%$ & 0 & 0 & N/A \\
Aggressiveness 0.6-0.8 & $1 \%$ & $3,492,200$ & $767,003,600$ & $0.46 \%$ \\
Aggressiveness 0.4-0.6 & $3 \%$ & $20,207,700$ & $3,255,798,040$ & $0.62 \%$ \\
Aggressiveness 0.2-0.4 & $4 \%$ & $-274,200$ & $4,867,563,536$ & $-0.01 \%$ \\
Aggressiveness 0.0-0.2 & $9 \%$ & $17,755,200$ & $11,681,200,344$ & $0.15 \%$ \\
\hline
\end{tabular}

Table 99: 8th July 2015 P\&L, 8316

- 8411

\begin{tabular}{|l|c|cc|c|}
\hline & Share & P\&L & Total volume in cash & P\&L vs total volume \\
\hline Server type B & $15 \%$ & $39,453,340$ & $24,121,638,711$ & $0.16 \%$ \\
others & $85 \%$ & $-39,453,340$ & $135,323,174,681$ & $-0.03 \%$ \\
\hline
\end{tabular}

Table 100: 8th July 2015 P\&L, 8411

\begin{tabular}{|l|c|cc|c|}
\hline Server type B & Share & P\&L & Total volume in cash & P\&L vs total volume \\
\hline Aggressiveness 0.8-1.0 & $0 \%$ & 0 & 0 & N/A \\
Aggressiveness 0.6-0.8 & $0 \%$ & $3,157,530$ & $761,826,127$ & $0.41 \%$ \\
Aggressiveness 0.4-0.6 & $3 \%$ & $31,082,340$ & $4,745,423,124$ & $0.65 \%$ \\
Aggressiveness 0.2-0.4 & $3 \%$ & $5,109,730$ & $4,117,529,988$ & $0.12 \%$ \\
Aggressiveness 0.0-0.2 & $9 \%$ & 119,680 & $14,489,841,600$ & $0.00 \%$ \\
\hline
\end{tabular}

Table 101: 8th July 2015 P\&L, 8411 


\begin{tabular}{|l|c|cc|c|}
\hline & Share & P\&L & Total volume in cash & P\&L vs total volume \\
\hline Server type B & $16 \%$ & $148,911,620$ & $27,365,561,740$ & $0.54 \%$ \\
others & $84 \%$ & $-148,911,620$ & $147,907,571,900$ & $-0.10 \%$ \\
\hline
\end{tabular}

Table 102: 8th July 2015 P\&L, 8306

\begin{tabular}{|l|c|cc|c|}
\hline Server type B & Share & P\&L & Total volume in cash & P\&L vs total volume \\
\hline Aggressiveness 0.8-1.0 & $0 \%$ & 0 & 0 & N/A \\
Aggressiveness 0.6-0.8 & $1 \%$ & $8,127,530$ & $1,598,529,416$ & $0.51 \%$ \\
Aggressiveness 0.4-0.6 & $4 \%$ & $84,078,450$ & $6,408,822,288$ & $1.31 \%$ \\
Aggressiveness 0.2-0.4 & $4 \%$ & $34,527,440$ & $6,543,215,712$ & $0.53 \%$ \\
Aggressiveness 0.0-0.2 & $7 \%$ & $22,178,200$ & $12,814,994,324$ & $0.17 \%$ \\
\hline
\end{tabular}

Table 103: 8th July 2015 P\&L, 8306

- 6758

\begin{tabular}{|l|c|cc|c|}
\hline & Share & P\&L & Total volume in cash & P\&L vs total volume \\
\hline Server type B & $12 \%$ & $26,040,250$ & $6,968,998,680$ & $0.37 \%$ \\
others & $88 \%$ & $-26,040,250$ & $50,760,615,640$ & $-0.05 \%$ \\
\hline
\end{tabular}

Table 104: 8th July 2015 P\&L, 6758

\begin{tabular}{|l|c|cc|c|}
\hline Server type B & Share & P\&L & Total volume in cash & P\&L vs total volume \\
\hline Aggressiveness 0.8-1.0 & $0 \%$ & 0 & 0 & N/A \\
Aggressiveness 0.6-0.8 & $0 \%$ & 175,750 & $76,374,440$ & $0.23 \%$ \\
Aggressiveness 0.4-0.6 & $1 \%$ & $6,001,800$ & $638,706,600$ & $0.94 \%$ \\
Aggressiveness 0.2-0.4 & $2 \%$ & $1,315,000$ & $1,298,365,480$ & $0.10 \%$ \\
Aggressiveness 0.0-0.2 & $9 \%$ & $18,511,000$ & $4,932,572,240$ & $0.38 \%$ \\
\hline
\end{tabular}

Table 105: 8th July 2015 P\&L, 6758

- 6762

\begin{tabular}{|l|c|cc|c|}
\hline & Share & P\&L & Total volume in cash & P\&L vs total volume \\
\hline Server type B & $18 \%$ & $4,487,000$ & $4,376,135,160$ & $0.10 \%$ \\
others & $82 \%$ & $-4,487,000$ & $20,479,961,052$ & $-0.02 \%$ \\
\hline
\end{tabular}

Table 106: 8th July 2015 P\&L, 6762

\begin{tabular}{|l|c|cc|c|}
\hline Server type B & Share & P\&L & Total volume in cash & P\&L vs total volume \\
\hline Aggressiveness 0.8-1.0 & $0 \%$ & 0 & 0 & N/A \\
Aggressiveness 0.6-0.8 & $0 \%$ & $-120,000$ & $101,055,330$ & $-0.12 \%$ \\
Aggressiveness 0.4-0.6 & $2 \%$ & $1,181,000$ & $459,582,066$ & $0.26 \%$ \\
Aggressiveness 0.2-0.4 & $5 \%$ & $1,939,000$ & $1,275,933,384$ & $0.15 \%$ \\
Aggressiveness 0.0-0.2 & $10 \%$ & $1,497,000$ & $2,532,534,444$ & $0.06 \%$ \\
\hline
\end{tabular}

Table 107: 8th July 2015 P\&L, 6762 


\begin{tabular}{|l|c|cc|c|}
\hline & Share & P\&L & Total volume in cash & P\&L vs total volume \\
\hline Server type B & $14 \%$ & $19,083,450$ & $4,876,461,656$ & $0.39 \%$ \\
others & $86 \%$ & $-19,083,450$ & $29,405,957,424$ & $-0.06 \%$ \\
\hline
\end{tabular}

Table 108: 8th July 2015 P\&L, 7270

\begin{tabular}{|l|c|cc|c|}
\hline Server type B & Share & P\&L & Total volume in cash & P\&L vs total volume \\
\hline Aggressiveness 0.8-1.0 & $0 \%$ & 0 & 0 & N/A \\
Aggressiveness 0.6-0.8 & $1 \%$ & $1,755,500$ & $198,440,274$ & $0.88 \%$ \\
Aggressiveness 0.4-0.6 & $2 \%$ & $5,836,550$ & $628,175,172$ & $0.93 \%$ \\
Aggressiveness 0.2-0.4 & $3 \%$ & $2,960,600$ & $961,975,368$ & $0.31 \%$ \\
Aggressiveness 0.0-0.2 & $9 \%$ & $8,529,900$ & $3,086,556,668$ & $0.28 \%$ \\
\hline
\end{tabular}

Table 109: 8th July 2015 P\&L, 7270

- 7267

\begin{tabular}{|l|c|cc|c|}
\hline & Share & P\&L & Total volume in cash & P\&L vs total volume \\
\hline Server type B & $16 \%$ & $11,231,650$ & $6,574,658,238$ & $0.17 \%$ \\
others & $84 \%$ & $-11,231,650$ & $34,158,475,356$ & $-0.03 \%$ \\
\hline
\end{tabular}

Table 110: 8th July 2015 P\&L, 7267

\begin{tabular}{|l|c|cc|c|}
\hline Server type B & Share & P\&L & Total volume in cash & P\&L vs total volume \\
\hline Aggressiveness 0.8-1.0 & $0 \%$ & 0 & 0 & N/A \\
Aggressiveness 0.6-0.8 & $1 \%$ & 735,600 & $215,746,709$ & $0.34 \%$ \\
Aggressiveness 0.4-0.6 & $2 \%$ & $6,433,650$ & $842,070,638$ & $0.76 \%$ \\
Aggressiveness 0.2-0.4 & $4 \%$ & $1,512,850$ & $1,705,444,811$ & $0.09 \%$ \\
Aggressiveness 0.0-0.2 & $9 \%$ & $2,525,650$ & $3,804,811,351$ & $0.07 \%$ \\
\hline
\end{tabular}

Table 111: 8th July 2015 P\&L, 7267

- 9984

\begin{tabular}{|l|c|cc|c|}
\hline & Share & P\&L & Total volume in cash & P\&L vs total volume \\
\hline Server type B & $12 \%$ & $7,311,900$ & $21,997,144,080$ & $0.03 \%$ \\
others & $88 \%$ & $-7,311,900$ & $160,852,988,400$ & $0.00 \%$ \\
\hline
\end{tabular}

Table 112: 8th July 2015 P\&L, 9984

\begin{tabular}{|l|c|cc|c|}
\hline Server type B & Share & P\&L & Total volume in cash & P\&L vs total volume \\
\hline Aggressiveness 0.8-1.0 & $0 \%$ & 0 & 0 & N/A \\
Aggressiveness 0.6-0.8 & $1 \%$ & $1,716,000$ & $1,031,224,560$ & $0.17 \%$ \\
Aggressiveness 0.4-0.6 & $2 \%$ & $9,589,500$ & $3,921,567,960$ & $0.24 \%$ \\
Aggressiveness 0.2-0.4 & $3 \%$ & $-8,188,300$ & $6,004,141,920$ & $-0.14 \%$ \\
Aggressiveness 0.0-0.2 & $6 \%$ & $4,170,300$ & $11,033,964,000$ & $0.04 \%$ \\
\hline
\end{tabular}

Table 113: 8th July 2015 P\&L, 9984 


\begin{tabular}{|l|c|cc|c|}
\hline & Share & P\&L & Total volume in cash & P\&L vs total volume \\
\hline Server type B & $11 \%$ & $38,378,500$ & $17,911,625,391$ & $0.21 \%$ \\
others & $89 \%$ & $-38,378,500$ & $140,712,941,853$ & $-0.03 \%$ \\
\hline
\end{tabular}

Table 114: 8th July 2015 P\&L, 7203

\begin{tabular}{|l|c|cc|c|}
\hline Server type B & Share & P\&L & Total volume in cash & P\&L vs total volume \\
\hline Aggressiveness 0.8-1.0 & $0 \%$ & 0 & 0 & N/A \\
Aggressiveness 0.6-0.8 & $0 \%$ & $-37,400$ & $88,802,109$ & $-0.04 \%$ \\
Aggressiveness 0.4-0.6 & $2 \%$ & $31,884,200$ & $3,588,885,234$ & $0.89 \%$ \\
Aggressiveness 0.2-0.4 & $3 \%$ & $-1,716,800$ & $4,485,706,533$ & $-0.04 \%$ \\
Aggressiveness 0.0-0.2 & $6 \%$ & $8,237,500$ & $9,743,431,401$ & $0.08 \%$ \\
\hline
\end{tabular}

Table 115: 8th July 2015 P\&L, 7203

- 2802

\begin{tabular}{|l|c|cc|c|}
\hline & Share & P\&L & Total volume in cash & P\&L vs total volume \\
\hline Server type B & $18 \%$ & 537,000 & $2,546,738,800$ & $0.02 \%$ \\
others & $82 \%$ & $-537,000$ & $11,485,241,200$ & $0.00 \%$ \\
\hline
\end{tabular}

Table 116: 8th July 2015 P\&L, 2802

\begin{tabular}{|l|c|cc|c|}
\hline Server type B & Share & P\&L & Total volume in cash & P\&L vs total volume \\
\hline Aggressiveness 0.8-1.0 & $0 \%$ & 0 & 0 & N/A \\
Aggressiveness 0.6-0.8 & $0 \%$ & $-256,500$ & $41,964,800$ & $-0.61 \%$ \\
Aggressiveness 0.4-0.6 & $2 \%$ & 136,000 & $257,034,400$ & $0.05 \%$ \\
Aggressiveness 0.2-0.4 & $5 \%$ & $1,447,500$ & $684,550,800$ & $0.21 \%$ \\
Aggressiveness 0.0-0.2 & $11 \%$ & $-790,000$ & $1,563,188,800$ & $-0.05 \%$ \\
\hline
\end{tabular}

Table 117: 8th July 2015 P\&L, 2802

\subsubsection{P\&L of Server type B by ticker, 1st September 2015}

\begin{tabular}{|l|cc|c|}
\hline ticker & start & end & daily return \\
\hline 6762 & 7360 & 7030 & $-4.48 \%$ \\
2802 & 2656 & 2550.5 & $-3.97 \%$ \\
7270 & 4230 & 4081 & $-3.52 \%$ \\
7267 & 3800 & 3679.5 & $-3.17 \%$ \\
8411 & 247 & 240 & $-2.83 \%$ \\
8306 & 797.1 & 777 & $-2.52 \%$ \\
9984 & 7030 & 6853 & $-2.52 \%$ \\
6758 & 3123.5 & 3051 & $-2.32 \%$ \\
8316 & 4913.5 & 4818 & $-1.94 \%$ \\
7203 & 7124 & 7000 & $-1.74 \%$ \\
\hline
\end{tabular}

Table 118: 1st September 2015 Daily return by ticker 


\begin{tabular}{|l|c|cc|c|}
\hline & Share & P\&L & Total volume in cash & P\&L vs total volume \\
\hline Server type B & $22 \%$ & $-9,198,000$ & $6,322,563,792$ & $-0.15 \%$ \\
others & $78 \%$ & $9,198,000$ & $23,018,195,584$ & $0.04 \%$ \\
\hline
\end{tabular}

Table 119: 1st September 2015 P\&L, 6762

\begin{tabular}{|l|c|cc|c|}
\hline Server type B & Share & P\&L & Total volume in cash & P\&L vs total volume \\
\hline Aggressiveness 0.8-1.0 & $0 \%$ & $-1,113,000$ & $120,184,512$ & $-0.93 \%$ \\
Aggressiveness 0.6-0.8 & $2 \%$ & $-4,404,000$ & $646,707,136$ & $-0.68 \%$ \\
Aggressiveness 0.4-0.6 & $1 \%$ & $-452,000$ & $367,707,376$ & $-0.12 \%$ \\
Aggressiveness 0.2-0.4 & $4 \%$ & $-1,540,000$ & $1,302,714,264$ & $-0.12 \%$ \\
Aggressiveness 0.0-0.2 & $13 \%$ & $-1,677,000$ & $3,882,388,968$ & $-0.04 \%$ \\
\hline
\end{tabular}

Table 120: 1st September 2015 P\&L, 6762

- 2802

\begin{tabular}{|l|c|cc|c|}
\hline & Share & P\&L & Total volume in cash & P\&L vs total volume \\
\hline Server type B & $24 \%$ & $17,019,500$ & $3,706,461,640$ & $0.46 \%$ \\
others & $76 \%$ & $-17,019,500$ & $11,622,666,840$ & $-0.15 \%$ \\
\hline
\end{tabular}

Table 121: 1st September 2015 P\&L, 2802

\begin{tabular}{|l|c|cc|c|}
\hline Server type B & Share & P\&L & Total volume in cash & P\&L vs total volume \\
\hline Aggressiveness 0.8-1.0 & $0 \%$ & 271,000 & $41,940,160$ & $0.65 \%$ \\
Aggressiveness 0.6-0.8 & $2 \%$ & 651,000 & $243,777,180$ & $0.27 \%$ \\
Aggressiveness 0.4-0.6 & $3 \%$ & $3,927,500$ & $495,418,140$ & $0.79 \%$ \\
Aggressiveness 0.2-0.4 & $8 \%$ & $10,179,000$ & $1,226,749,680$ & $0.83 \%$ \\
Aggressiveness 0.0-0.2 & $11 \%$ & $2,007,000$ & $1,640,908,760$ & $0.12 \%$ \\
\hline
\end{tabular}

Table 122: 1st September 2015 P\&L, 2802

- 7270

\begin{tabular}{|l|c|cc|c|}
\hline & Share & P\&L & Total volume in cash & P\&L vs total volume \\
\hline Server type B & $24 \%$ & $6,811,350$ & $9,242,261,532$ & $0.07 \%$ \\
others & $76 \%$ & $-6,811,350$ & $28,493,960,044$ & $-0.02 \%$ \\
\hline
\end{tabular}

Table 123: 1st September 2015 P\&L, 7270

\begin{tabular}{|l|c|cc|c|}
\hline Server type B & Share & P\&L & Total volume in cash & P\&L vs total volume \\
\hline Aggressiveness 0.8-1.0 & $1 \%$ & 757,650 & $291,742,776$ & $0.26 \%$ \\
Aggressiveness 0.6-0.8 & $2 \%$ & $2,446,500$ & $809,149,836$ & $0.30 \%$ \\
Aggressiveness 0.4-0.6 & $3 \%$ & $7,286,150$ & $1,099,645,848$ & $0.66 \%$ \\
Aggressiveness 0.2-0.4 & $6 \%$ & 276,500 & $2,446,566,556$ & $0.01 \%$ \\
Aggressiveness 0.0-0.2 & $12 \%$ & $-4,085,150$ & $4,577,701,820$ & $-0.09 \%$ \\
\hline
\end{tabular}

Table 124: 1st September 2015 P\&L, 7270 


\begin{tabular}{|l|c|cc|c|}
\hline & Share & P\&L & Total volume in cash & P\&L vs total volume \\
\hline Server type B & $24 \%$ & $7,575,150$ & $8,851,859,280$ & $0.09 \%$ \\
others & $76 \%$ & $-7,575,150$ & $27,814,061,874$ & $-0.03 \%$ \\
\hline
\end{tabular}

Table 125: 1st September 2015 P\&L, 7267

\begin{tabular}{|l|c|cc|c|}
\hline Server type B & Share & P\&L & Total volume in cash & P\&L vs total volume \\
\hline Aggressiveness 0.8-1.0 & $1 \%$ & 539,450 & $240,669,238$ & $0.22 \%$ \\
Aggressiveness 0.6-0.8 & $2 \%$ & 541,250 & $744,360,894$ & $0.07 \%$ \\
Aggressiveness 0.4-0.6 & $7 \%$ & $3,620,900$ & $2,613,831,848$ & $0.14 \%$ \\
Aggressiveness 0.2-0.4 & $4 \%$ & $-4,688,500$ & $1,289,405,933$ & $-0.36 \%$ \\
Aggressiveness 0.0-0.2 & $11 \%$ & $7,652,750$ & $3,930,061,597$ & $0.19 \%$ \\
\hline
\end{tabular}

Table 126: 1st September 2015 P\&L, 7267

- 8411

\begin{tabular}{|l|c|cc|c|}
\hline & Share & P\&L & Total volume in cash & P\&L vs total volume \\
\hline Server type B & $19 \%$ & $44,960,260$ & $22,059,147,366$ & $0.20 \%$ \\
others & $81 \%$ & $-44,960,260$ & $94,046,785,812$ & $-0.05 \%$ \\
\hline
\end{tabular}

Table 127: 1st September 2015 P\&L, 8411

\begin{tabular}{|l|c|cc|c|}
\hline Server type B & Share & P\&L & Total volume in cash & P\&L vs total volume \\
\hline Aggressiveness 0.8-1.0 & $1 \%$ & $2,253,690$ & $1,038,211,651$ & $0.22 \%$ \\
Aggressiveness 0.6-0.8 & $1 \%$ & $9,616,690$ & $792,372,741$ & $1.21 \%$ \\
Aggressiveness 0.4-0.6 & $3 \%$ & $20,764,210$ & $3,784,454,434$ & $0.55 \%$ \\
Aggressiveness 0.2-0.4 & $7 \%$ & $3,221,730$ & $8,640,468,677$ & $0.04 \%$ \\
Aggressiveness 0.0-0.2 & $7 \%$ & $9,051,320$ & $7,784,890,679$ & $0.12 \%$ \\
\hline
\end{tabular}

Table 128: 1st September 2015 P\&L, 8411

- 8306

\begin{tabular}{|l|c|cc|c|}
\hline & Share & P\&L & Total volume in cash & P\&L vs total volume \\
\hline Server type B & $19 \%$ & $20,878,480$ & $22,629,179,246$ & $0.09 \%$ \\
others & $81 \%$ & $-20,878,480$ & $97,354,890,922$ & $-0.02 \%$ \\
\hline
\end{tabular}

Table 129: 1st September 2015 P\&L, 8306

\begin{tabular}{|l|c|cc|c|}
\hline Server type B & Share & P\&L & Total volume in cash & P\&L vs total volume \\
\hline Aggressiveness 0.8-1.0 & $3 \%$ & $3,353,390$ & $3,285,602,956$ & $0.10 \%$ \\
Aggressiveness 0.6-0.8 & $1 \%$ & $8,258,060$ & $1,234,048,488$ & $0.67 \%$ \\
Aggressiveness 0.4-0.6 & $4 \%$ & $23,592,220$ & $5,314,497,008$ & $0.44 \%$ \\
Aggressiveness 0.2-0.4 & $4 \%$ & $-18,892,980$ & $5,393,336,372$ & $-0.35 \%$ \\
Aggressiveness 0.0-0.2 & $6 \%$ & $4,567,790$ & $7,401,694,422$ & $0.06 \%$ \\
\hline
\end{tabular}

Table 130: 1st September 2015 P\&L, 8306 


\begin{tabular}{|l|c|cc|c|}
\hline & Share & P\&L & Total volume in cash & P\&L vs total volume \\
\hline Server type B & $22 \%$ & $18,751,000$ & $22,673,152,892$ & $0.08 \%$ \\
others & $78 \%$ & $-18,751,000$ & $78,836,135,872$ & $-0.02 \%$ \\
\hline
\end{tabular}

Table 131: 1st September 2015 P\&L, 9984

\begin{tabular}{|l|c|cc|c|}
\hline Server type B & Share & P\&L & Total volume in cash & P\&L vs total volume \\
\hline Aggressiveness 0.8-1.0 & $1 \%$ & $-236,300$ & $1,218,643,842$ & $-0.02 \%$ \\
Aggressiveness 0.6-0.8 & $3 \%$ & $1,370,900$ & $3,259,421,698$ & $0.04 \%$ \\
Aggressiveness 0.4-0.6 & $3 \%$ & $5,252,100$ & $3,280,910,867$ & $0.16 \%$ \\
Aggressiveness 0.2-0.4 & $6 \%$ & $-6,866,500$ & $6,539,639,366$ & $-0.10 \%$ \\
Aggressiveness 0.0-0.2 & $8 \%$ & $19,364,700$ & $8,274,716,463$ & $0.23 \%$ \\
\hline
\end{tabular}

Table 132: 1st September 2015 P\&L, 9984

- 6758

\begin{tabular}{|l|c|cc|c|}
\hline & Share & P\&L & Total volume in cash & P\&L vs total volume \\
\hline Server type B & $21 \%$ & $24,449,750$ & $13,555,727,319$ & $0.18 \%$ \\
others & $79 \%$ & $-24,449,750$ & $49,696,660,665$ & $-0.05 \%$ \\
\hline
\end{tabular}

Table 133: 1st September 2015 P\&L, 6758

\begin{tabular}{|l|c|cc|c|}
\hline Server type B & Share & P\&L & Total volume in cash & P\&L vs total volume \\
\hline Aggressiveness 0.8-1.0 & $2 \%$ & $2,319,550$ & $1,533,530,730$ & $0.15 \%$ \\
Aggressiveness 0.6-0.8 & $1 \%$ & $-1,511,950$ & $674,691,309$ & $-0.22 \%$ \\
Aggressiveness 0.4-0.6 & $3 \%$ & 468,600 & $2,209,777,344$ & $0.02 \%$ \\
Aggressiveness 0.2-0.4 & $6 \%$ & $11,777,100$ & $3,621,994,284$ & $0.33 \%$ \\
Aggressiveness 0.0-0.2 & $9 \%$ & $11,442,350$ & $5,452,277,208$ & $0.21 \%$ \\
\hline
\end{tabular}

Table 134: 1st September 2015 P\&L, 6758

- 8316

\begin{tabular}{|l|c|cc|c|}
\hline & Share & P\&L & Total volume in cash & P\&L vs total volume \\
\hline Server type B & $24 \%$ & $14,032,600$ & $22,308,564,957$ & $0.06 \%$ \\
others & $76 \%$ & $-14,032,600$ & $70,770,301,249$ & $-0.02 \%$ \\
\hline
\end{tabular}

Table 135: 1st September 2015 P\&L, 8316

\begin{tabular}{|l|c|cc|c|}
\hline Server type B & Share & P\&L & Total volume in cash & P\&L vs total volume \\
\hline Aggressiveness 0.8-1.0 & $3 \%$ & 420,400 & $2,787,168,991$ & $0.02 \%$ \\
Aggressiveness 0.6-0.8 & $1 \%$ & $3,917,000$ & $1,377,252,475$ & $0.28 \%$ \\
Aggressiveness 0.4-0.6 & $2 \%$ & $5,455,900$ & $2,279,657,548$ & $0.24 \%$ \\
Aggressiveness 0.2-0.4 & $5 \%$ & $-15,140,350$ & $4,805,514,211$ & $-0.32 \%$ \\
Aggressiveness 0.0-0.2 & $12 \%$ & $19,394,250$ & $10,941,966,212$ & $0.18 \%$ \\
\hline
\end{tabular}

Table 136: 1st September 2015 P\&L, 8316 


\begin{tabular}{|l|c|cc|c|}
\hline & Share & P\&L & Total volume in cash & P\&L vs total volume \\
\hline Server type B & $23 \%$ & $61,804,500$ & $32,759,161,960$ & $0.19 \%$ \\
others & $77 \%$ & $-61,804,500$ & $107,199,371,192$ & $-0.06 \%$ \\
\hline
\end{tabular}

Table 137: 1st September 2015 P\&L, 7203

\begin{tabular}{|l|c|cc|c|}
\hline Server type B & Share & P\&L & Total volume in cash & P\&L vs total volume \\
\hline Aggressiveness 0.8-1.0 & $0 \%$ & 987,500 & $636,973,864$ & $0.16 \%$ \\
Aggressiveness 0.6-0.8 & $1 \%$ & $6,160,600$ & $1,813,852,160$ & $0.34 \%$ \\
Aggressiveness 0.4-0.6 & $5 \%$ & $24,728,500$ & $7,307,840,304$ & $0.34 \%$ \\
Aggressiveness 0.2-0.4 & $6 \%$ & $3,663,500$ & $8,301,916,312$ & $0.04 \%$ \\
Aggressiveness 0.0-0.2 & $10 \%$ & $26,222,400$ & $14,593,007,456$ & $0.18 \%$ \\
\hline
\end{tabular}

Table 138: 1st September 2015 P\&L, 7203

\subsection{P\&L of Server type A}

Tables 141-151 \& 154-164 show the P\&L of Server type A made in trading of the 10 representative stocks on 8th July and 1st September 2015, which correspond to the P\&L of Server type B in Tables 93-138 in Section 4.3. We observe that Server type A also makes profits constantly for 8 out of 10 stocks on 8th July 2015 and 10 out of 10 stocks on 1st September 2015.

In addition, we calculate selling/buying execution shares of Server type A in price falls/rises from local high/low prices, which corresponds to the analysis in Section 3.1 and Appendix A. Tables 139, 140, 152 \& 153 show selling/buying shares of Server type A in the 10 second intervals with price falls/rises from a local high/low price, which is the high/low price in 5 minutes before and after the interval for 8th July and 1st September 2015. These tables also show the shares in the price falls/rises from the other prices.

We observe that the selling/buying shares of Server type A in the small price falls/rises from the local high/low prices are high compared to those in the small price falls/rises from the other prices for both 8th July and 1st September 2015, while it is not the case with the 10 second intervals with price falls greater than 10 bps on 8th July 2015. This implies that Server type A captures the tops and bottoms particularly in the small price moves in making the profits, which may not be done by the other server types.

\subsubsection{P\&L of Server type A by ticker, 8th July 2015}

\begin{tabular}{|l|c|c|c|c|}
\hline & Selling share (fall from a local high price) & \# of times & Selling share (other fall cases) & \# of times \\
\hline Negative return greater than $0.10 \%$ & $25 \%$ & 67 & 423 \\
Negative return less than $0.10 \%$ & $37 \%$ & 147 & $32 \%$ \\
\hline
\end{tabular}

Table 139: Execution shares of Server type A in price falls, falls from a local high price vs other cases, 8th July 2015 


\begin{tabular}{|c|c|c|c|c|}
\hline & Buying share (rise from a local low price) & \# of times & Buying share (other rise cases) & \# of times \\
\hline Positive return greater than $0.10 \%$ & $52 \%$ & 48 & $37 \%$ & 365 \\
\hline Positive return less than $0.10 \%$ & $46 \%$ & 158 & $36 \%$ & 5138 \\
\hline
\end{tabular}

Table 140: Execution shares of Server type A in price rises, rises from a local low price vs other cases, 8th July 2015

\begin{tabular}{|c|c|cc|c|}
\hline & Share & P\&L & Total volume in cash & P\&L vs total volume \\
\hline Server type A & $23 \%$ & $66,698,980$ & $221,161,827,600$ & $0.03 \%$ \\
\hline
\end{tabular}

Table 141: 8th July 2015 P\&L

\begin{tabular}{|c|c|cc|c|}
\hline & Share & P\&L & Total volume in cash & P\&L vs total volume \\
\hline Server type A & $27 \%$ & $1,573,500$ & $3,850,166,500$ & $0.04 \%$ \\
\hline
\end{tabular}

Table 142: 8th July 2015 P\&L, 2802

\begin{tabular}{|c|c|cc|c|}
\hline & Share & P\&L & Total volume in cash & P\&L vs total volume \\
\hline Server type A & $21 \%$ & $12,837,300$ & $38,505,061,500$ & $0.03 \%$ \\
\hline
\end{tabular}

Table 143: 8th July 2015 P\&L, 9984

\begin{tabular}{|c|c|cc|c|}
\hline & Share & P\&L & Total volume in cash & P\&L vs total volume \\
\hline Server type A & $29 \%$ & $4,974,800$ & $11,857,364,400$ & $0.04 \%$ \\
\hline
\end{tabular}

Table 144: 8th July 2015 P\&L, 7267

\begin{tabular}{|c|c|cc|c|}
\hline & Share & P\&L & Total volume in cash & P\&L vs total volume \\
\hline Server type A & $27 \%$ & $1,225,100$ & $9,367,624,300$ & $0.01 \%$ \\
\hline
\end{tabular}

Table 145: 8th July 2015 P\&L, 7270

\begin{tabular}{|c|c|cc|c|}
\hline & Share & P\&L & Total volume in cash & P\&L vs total volume \\
\hline Server type A & $26 \%$ & $-5,698,000$ & $6,414,408,000$ & $-0.09 \%$ \\
\hline
\end{tabular}

Table 146: 8th July 2015 P\&L, 6762

\begin{tabular}{|c|c|cc|c|}
\hline & Share & P\&L & Total volume in cash & P\&L vs total volume \\
\hline Server type A & $24 \%$ & $9,560,400$ & $14,044,861,500$ & $0.07 \%$ \\
\hline
\end{tabular}

Table 147: 8th July 2015 P\&L, 6758 


\begin{tabular}{|c|c|cc|c|}
\hline & Share & P\&L & Total volume in cash & P\&L vs total volume \\
\hline Server type A & $19 \%$ & $8,952,220$ & $33,853,746,090$ & $0.03 \%$ \\
\hline
\end{tabular}

Table 148: 8th July 2015 P\&L, 8306

\begin{tabular}{|c|c|cc|c|}
\hline & Share & P\&L & Total volume in cash & P\&L vs total volume \\
\hline Server type A & $19 \%$ & $17,096,260$ & $30,059,611,910$ & $0.06 \%$ \\
\hline
\end{tabular}

Table 149: 8th July 2015 P\&L, 8411

\begin{tabular}{|c|c|cc|c|}
\hline & Share & P\&L & Total volume in cash & P\&L vs total volume \\
\hline Server type A & $24 \%$ & $-3,859,700$ & $31,361,003,100$ & $-0.01 \%$ \\
\hline
\end{tabular}

Table 150: 8th July 2015 P\&L, 8316

\begin{tabular}{|c|c|cc|c|}
\hline & Share & P\&L & Total volume in cash & P\&L vs total volume \\
\hline Server type A & $26 \%$ & $20,037,100$ & $41,847,980,300$ & $0.05 \%$ \\
\hline
\end{tabular}

Table 151: 8th July 2015 P\&L, 7203

\subsubsection{P\&L of Server type A by ticker, 1st September 2015}

\begin{tabular}{|c|c|c|c|c|}
\hline & Selling share (fall from a local high price) & \# of times & Selling share (other fall cases) & \# of times \\
\hline Negative return greater than $0.10 \%$ & $35 \%$ & 58 & $32 \%$ & 654 \\
\hline Negative return less than $0.10 \%$ & $37 \%$ & 118 & $31 \%$ & 5676 \\
\hline
\end{tabular}

Table 152: Execution shares of Server type A in price falls, falls from a local high price vs other cases, 1st September 2015

\begin{tabular}{|l|c|c|c|c|}
\hline & Buying share (rise from a local low price) & \# of times & Buying share (other rise cases) & \# of times \\
\hline Positive return greater than $0.10 \%$ & $40 \%$ & 60 & 594 \\
Positive return less than $0.10 \%$ & $41 \%$ & 122 & $35 \%$ \\
\hline
\end{tabular}

Table 153: Execution shares of Server type A in price rises, rises from a local low price vs other cases, 1st September 2015

\begin{tabular}{|c|c|cc|c|}
\hline & Share & P\&L & Total volume in cash & P\&L vs total volume \\
\hline Server type A & $22 \%$ & $117,688,090$ & $164,490,496,170$ & $0.07 \%$ \\
\hline
\end{tabular}

Table 154: 1st September 2015 P\&L

\begin{tabular}{|c|c|cc|c|}
\hline & Share & P\&L & Total volume in cash & P\&L vs total volume \\
\hline Server type A & $23 \%$ & $9,449,900$ & $23,379,046,900$ & $0.04 \%$ \\
\hline
\end{tabular}

Table 155: 1st September 2015 P\&L, 9984 


\begin{tabular}{|c|c|cc|c|}
\hline & Share & P\&L & Total volume in cash & P\&L vs total volume \\
\hline Server type A & $27 \%$ & $10,954,690$ & $31,876,490,170$ & $0.03 \%$ \\
\hline
\end{tabular}

Table 156: 1st September 2015 P\&L, 8411

\begin{tabular}{|c|c|cc|c|}
\hline & Share & P\&L & Total volume in cash & P\&L vs total volume \\
\hline Server type A & $22 \%$ & $15,518,250$ & $20,205,512,350$ & $0.08 \%$ \\
\hline
\end{tabular}

Table 157: 1st September 2015 P\&L, 8316

\begin{tabular}{|c|c|cc|c|}
\hline & Share & P\&L & Total volume in cash & P\&L vs total volume \\
\hline Server type A & $19 \%$ & $26,537,150$ & $22,322,593,550$ & $0.12 \%$ \\
\hline
\end{tabular}

Table 158: 1st September 2015 P\&L, 8306

\begin{tabular}{|c|c|cc|c|}
\hline & Share & P\&L & Total volume in cash & P\&L vs total volume \\
\hline Server type A & $23 \%$ & $4,007,900$ & $8,577,699,100$ & $0.05 \%$ \\
\hline
\end{tabular}

Table 159: 1st September 2015 P\&L, 7270

\begin{tabular}{|c|c|cc|c|}
\hline & Share & P\&L & Total volume in cash & P\&L vs total volume \\
\hline Server type A & $25 \%$ & $9,991,800$ & $9,194,772,700$ & $0.11 \%$ \\
\hline
\end{tabular}

Table 160: 1st September 2015 P\&L, 7267

\begin{tabular}{|c|c|cc|c|}
\hline & Share & P\&L & Total volume in cash & P\&L vs total volume \\
\hline Server type A & $19 \%$ & $25,138,600$ & $26,373,838,800$ & $0.10 \%$ \\
\hline
\end{tabular}

Table 161: 1st September 2015 P\&L, 7203

\begin{tabular}{|c|c|cc|c|}
\hline & Share & P\&L & Total volume in cash & P\&L vs total volume \\
\hline Server type A & $21 \%$ & 528,000 & $6,273,430,000$ & $0.01 \%$ \\
\hline
\end{tabular}

Table 162: 1st September 2015 P\&L, 6762

\begin{tabular}{|c|c|cc|c|}
\hline & Share & P\&L & Total volume in cash & P\&L vs total volume \\
\hline Server type A & $19 \%$ & $5,021,800$ & $12,246,886,600$ & $0.04 \%$ \\
\hline
\end{tabular}

Table 163: 1st September 2015 P\&L, 6758 


\begin{tabular}{|c|c|cc|c|}
\hline & Share & P\&L & Total volume in cash & P\&L vs total volume \\
\hline Server type A & $26 \%$ & $10,540,000$ & $4,040,226,000$ & $0.26 \%$ \\
\hline
\end{tabular}

Table 164: 1st September 2015 P\&L, 2802

\section{Conclusion}

We have observed that various sorts of servers trade and place orders differently in the market movements. Particularly, Server type A follows the short-term market trend, selling in the price falls and buying in the price rises, which accelerates the market movements. Moreover, Server type A places large orders along with the price movements, which opposes to rebound of the stock price. We have also found that while Server type $\mathrm{B}$ cancels its buying/selling orders in the rapid price falls/rises, it has a constant share of buying/selling orders in such a situation. The other types, Server types C \& D consistently buy/sell and place orders regardless of the market situation. Furthermore, we have investigated shares of the executed orders by the server type in the large swings and roundabout of the Nikkei 225 index, which occurred in July, August, and September 2015, and the two patterns have been observed. The first one is that the market is highly liquid and Server type $\mathrm{C}$ has the largest share of buying volume in the price rebound. The second one is that the market is thin and the stock prices rebound accompanying the large price fluctuations due to the trading of Server type A. In both cases, in the price falls of the swing and the roundabouts, Server type A has the largest share of selling volume. Also, we have investigated at which level Server type B buys the stocks in the price falls. We have observed that passive servers are the main buyers and they trade at low levels. Moreover, in the price falling days, Server types B\&A make profits from the other server types. In particular, the aggressive servers earn most of the profit of Server type B.

\section{Acknowledgment}

We would like to express our gratitude to JPX for supplying the order data in TSE.

\section{References}

[1] Riordan, R., Storkenmaier, A. (2012). Latency, liquidity and price discovery. Journal of Financial Markets, 15(4), 416-437.

[2] Menkveld, A. J. (2013). High frequency trading and the new market makers. Journal of Financial Markets, 16(4), 712-740.

[3] O’ Hara, M. (2015). High frequency market microstructure. Journal of Financial Economics, 116(2), 257-270.

[4] Kirilenko, A. A., Kyle, A. S., Samadi, M., Tuzun, T. (2015). The flash crash: The impact of high frequency trading on an electronic market. Available at SSRN 1686004. 
[5] Boehmer, E., Jones, C. M., Zhang, X. (2013). Shackling short sellers: The 2008 shorting ban. Review of Financial Studies, hht017.

[6] Hasbrouck, J., Saar, G. (2013). Low-latency trading. Journal of Financial Markets, 16(4), 646-679.

[7] Carrion, A. (2013). Very fast money: High-frequency trading on the NASDAQ. Journal of Financial Markets, 16(4), 680-711.

[8] Brogaard, J., Hendershott, T., Riordan, R. (2014). High-frequency trading and price discovery. Review of Financial Studies, 27(8), 2267-2306.

[9] Hosaka, G. (2014). Analysis of high-frequency trading at Tokyo Stock Exchange. JPX Working paper, Japan Exchange Group. Available at http://www. jpx. co. jp/generalinformation/research-study/wp. html (accessed March 25, 2015).

[10] Labadie, M., Lehalle, C. A. (2010). Optimal algorithmic trading and market microstructure.

[11] Kearns, M., Nevmyvaka, Y. (2013). Machine learning for market microstructure and high frequency trading. High Frequency Trading-New Realities for Traders, Markets and Regulators, David Easley, Marcos Lopez de Prado and Maureen O ' Hara editors, Risk Books.

\section{A Changes in order volume for the other trading dates}

This section provides the results of the same analysis as Section 3 for the other dates which are also investigated in Section 4.

\section{A.0.1 4th September}

\begin{tabular}{|l|ccc|}
\hline & Executed order volume & Change in order volume & Price renewal \\
\hline Server type A & $13 \%$ & $26 \%$ & 1.06 \\
Server type B & $25 \%$ & $-56 \%$ & 0.47 \\
Server type C & $49 \%$ & $32 \%$ & 2.41 \\
Server type D & $13 \%$ & $1 \%$ & 0.54 \\
\hline
\end{tabular}

Table 165: Intervals with a negative return greater than $0.10 \%$, buy orders

\begin{tabular}{|l|ccc|}
\hline & Executed order volume & Change in order volume & Price renewal \\
\hline Server type A & $39 \%$ & $156 \%$ & 2.76 \\
Server type B & $18 \%$ & $154 \%$ & 1.09 \\
Server type C & $33 \%$ & $74 \%$ & 4.38 \\
Server type D & $10 \%$ & $22 \%$ & 1.18 \\
\hline
\end{tabular}

Table 166: Intervals with a negative return greater than $0.10 \%$, sell orders 


\begin{tabular}{|c|ccc|}
\hline & Executed order volume & Change in order volume & Price renewal \\
\hline Server type A & $18 \%$ & $28 \%$ & 0.76 \\
Server type B & $18 \%$ & $-18 \%$ & 0.28 \\
Server type C & $51 \%$ & $33 \%$ & 1.81 \\
Server type D & $13 \%$ & $9 \%$ & 0.44 \\
\hline
\end{tabular}

Table 167: Intervals with a negative return less than $0.10 \%$, buy orders

\begin{tabular}{|l|ccc|}
\hline & Executed order volume & Change in order volume & Price renewal \\
\hline Server type A & $33 \%$ & $101 \%$ & 1.46 \\
Server type B & $18 \%$ & $63 \%$ & 0.64 \\
Server type C & $40 \%$ & $67 \%$ & 2.44 \\
Server type D & $9 \%$ & $14 \%$ & 0.54 \\
\hline
\end{tabular}

Table 168: Intervals with a negative return less than $0.10 \%$, sell orders

\begin{tabular}{|l|ccc|}
\hline & Executed order volume & Change in order volume & Price renewal \\
\hline Server type A & $35 \%$ & $127 \%$ & 1.56 \\
Server type B & $13 \%$ & $46 \%$ & 0.41 \\
Server type C & $42 \%$ & $63 \%$ & 2.41 \\
Server type D & $10 \%$ & $17 \%$ & 0.60 \\
\hline
\end{tabular}

Table 169: Intervals with a positive return less than $0.10 \%$, buy orders

\begin{tabular}{|l|ccc|}
\hline & Executed order volume & Change in order volume & Price renewal \\
\hline Server type A & $18 \%$ & $20 \%$ & 0.81 \\
Server type B & $22 \%$ & $-29 \%$ & 0.35 \\
Server type C & $50 \%$ & $38 \%$ & 1.69 \\
Server type D & $10 \%$ & $5 \%$ & 0.35 \\
\hline
\end{tabular}

Table 170: Intervals with a positive return less than $0.10 \%$, sell orders

\begin{tabular}{|l|ccc|}
\hline & Executed order volume & Change in order volume & Price renewal \\
\hline Server type A & $42 \%$ & $169 \%$ & 2.92 \\
Server type B & $15 \%$ & $105 \%$ & 1.07 \\
Server type C & $36 \%$ & $67 \%$ & 3.31 \\
Server type D & $8 \%$ & $17 \%$ & 0.81 \\
\hline
\end{tabular}

Table 171: Intervals with a positive return greater than $0.10 \%$, buy orders

\begin{tabular}{|l|ccc|}
\hline & Executed order volume & Change in order volume & Price renewal \\
\hline Server type A & $15 \%$ & $23 \%$ & 1.09 \\
Server type B & $26 \%$ & $-94 \%$ & 0.50 \\
Server type C & $48 \%$ & $24 \%$ & 2.04 \\
Server type D & $11 \%$ & $1 \%$ & 0.47 \\
\hline
\end{tabular}

Table 172: Intervals with a positive return greater than $0.10 \%$, sell orders 


\section{A.0.2 8th September}

\begin{tabular}{|l|ccc|}
\hline & Executed order volume & Change in order volume & Price renewal \\
\hline Server type A & $14 \%$ & $21 \%$ & 0.79 \\
Server type B & $25 \%$ & $-43 \%$ & 0.35 \\
Server type C & $50 \%$ & $38 \%$ & 1.52 \\
Server type D & $11 \%$ & $2 \%$ & 0.24 \\
\hline
\end{tabular}

Table 173: Intervals with a negative return greater than $0.10 \%$, buy orders

\begin{tabular}{|c|ccc|}
\hline & Executed order volume & Change in order volume & Price renewal \\
\hline Server type A & $35 \%$ & $124 \%$ & 2.24 \\
Server type B & $15 \%$ & $112 \%$ & 0.87 \\
Server type C & $42 \%$ & $76 \%$ & 2.99 \\
Server type D & $8 \%$ & $29 \%$ & 0.69 \\
\hline
\end{tabular}

Table 174: Intervals with a negative return greater than $0.10 \%$, sell orders

\begin{tabular}{|l|ccc|}
\hline & Executed order volume & Change in order volume & Price renewal \\
\hline Server type A & $19 \%$ & $29 \%$ & 0.57 \\
Server type B & $18 \%$ & $-28 \%$ & 0.19 \\
Server type C & $52 \%$ & $39 \%$ & 1.30 \\
Server type D & $11 \%$ & $10 \%$ & 0.22 \\
\hline
\end{tabular}

Table 175: Intervals with a negative return less than $0.10 \%$, buy orders

\begin{tabular}{|l|ccc|}
\hline & Executed order volume & Change in order volume & Price renewal \\
\hline Server type A & $36 \%$ & $114 \%$ & 1.22 \\
Server type B & $15 \%$ & $76 \%$ & 0.49 \\
Server type C & $39 \%$ & $74 \%$ & 1.83 \\
Server type D & $10 \%$ & $29 \%$ & 0.43 \\
\hline
\end{tabular}

Table 176: Intervals with a negative return less than $0.10 \%$, sell orders

\begin{tabular}{|c|ccc|}
\hline & Executed order volume & Change in order volume & Price renewal \\
\hline Server type A & $38 \%$ & $126 \%$ & 1.38 \\
Server type B & $13 \%$ & $66 \%$ & 0.44 \\
Server type C & $40 \%$ & $82 \%$ & 1.83 \\
Server type D & $9 \%$ & $24 \%$ & 0.42 \\
\hline
\end{tabular}

Table 177: Intervals with a positive return less than $0.10 \%$, buy orders

\begin{tabular}{|l|ccc|}
\hline & Executed order volume & Change in order volume & Price renewal \\
\hline Server type A & $18 \%$ & $25 \%$ & 0.53 \\
Server type B & $18 \%$ & $-22 \%$ & 0.23 \\
Server type C & $53 \%$ & $39 \%$ & 1.24 \\
Server type D & $11 \%$ & $9 \%$ & 0.26 \\
\hline
\end{tabular}

Table 178: Intervals with a positive return less than $0.10 \%$, sell orders 


\begin{tabular}{|c|ccc|}
\hline & Executed order volume & Change in order volume & Price renewal \\
\hline Server type A & $39 \%$ & $146 \%$ & 2.16 \\
Server type B & $16 \%$ & $129 \%$ & 0.78 \\
Server type C & $37 \%$ & $74 \%$ & 2.71 \\
Server type D & $8 \%$ & $16 \%$ & 0.69 \\
\hline
\end{tabular}

Table 179: Intervals with a positive return greater than $0.10 \%$, buy orders

\begin{tabular}{|l|ccc|}
\hline & Executed order volume & Change in order volume & Price renewal \\
\hline Server type A & $15 \%$ & $19 \%$ & 0.64 \\
Server type B & $31 \%$ & $-74 \%$ & 0.37 \\
Server type C & $45 \%$ & $25 \%$ & 1.37 \\
Server type D & $10 \%$ & $2 \%$ & 0.28 \\
\hline
\end{tabular}

Table 180: Intervals with a positive return greater than $0.10 \%$, sell orders

\section{A.0.3 9th September}

\begin{tabular}{|l|ccc|}
\hline & Executed order volume & Change in order volume & Price renewal \\
\hline Server type A & $17 \%$ & $23 \%$ & 1.18 \\
Server type B & $25 \%$ & $-41 \%$ & 0.42 \\
Server type C & $45 \%$ & $29 \%$ & 1.84 \\
Server type D & $13 \%$ & $8 \%$ & 0.39 \\
\hline
\end{tabular}

Table 181: Intervals with a positive return greater than $0.10 \%$, buy orders

\begin{tabular}{|l|ccc|}
\hline & Executed order volume & Change in order volume & Price renewal \\
\hline Server type A & $28 \%$ & $95 \%$ & 2.02 \\
Server type B & $14 \%$ & $97 \%$ & 0.91 \\
Server type C & $45 \%$ & $75 \%$ & 3.83 \\
Server type D & $13 \%$ & $24 \%$ & 1.20 \\
\hline
\end{tabular}

Table 182: Intervals with a negative return greater than $0.10 \%$, sell orders

\begin{tabular}{|l|ccc|}
\hline & Executed order volume & Change in order volume & Price renewal \\
\hline Server type A & $17 \%$ & $29 \%$ & 0.73 \\
Server type B & $15 \%$ & $-20 \%$ & 0.24 \\
Server type C & $54 \%$ & $43 \%$ & 1.54 \\
Server type D & $14 \%$ & $5 \%$ & 0.34 \\
\hline
\end{tabular}

Table 183: Intervals with a negative return less than $0.10 \%$, buy orders

\begin{tabular}{|l|ccc|}
\hline & Executed order volume & Change in order volume & Price renewal \\
\hline Server type A & $30 \%$ & $100 \%$ & 1.12 \\
Server type B & $12 \%$ & $45 \%$ & 0.47 \\
Server type C & $47 \%$ & $75 \%$ & 2.67 \\
Server type D & $11 \%$ & $16 \%$ & 0.65 \\
\hline
\end{tabular}

Table 184: Intervals with a negative return less than $0.10 \%$, sell orders 


\begin{tabular}{|c|ccc|}
\hline & Executed order volume & Change in order volume & Price renewal \\
\hline Server type A & $37 \%$ & $94 \%$ & 1.71 \\
Server type B & $11 \%$ & $42 \%$ & 0.44 \\
Server type C & $42 \%$ & $71 \%$ & 2.27 \\
Server type D & $11 \%$ & $24 \%$ & 0.53 \\
\hline
\end{tabular}

Table 185: Intervals with a positive return less than $0.10 \%$, buy orders

\begin{tabular}{|l|ccc|}
\hline & Executed order volume & Change in order volume & Price renewal \\
\hline Server type A & $15 \%$ & $22 \%$ & 0.61 \\
Server type B & $15 \%$ & $-14 \%$ & 0.27 \\
Server type C & $57 \%$ & $33 \%$ & 2.08 \\
Server type D & $13 \%$ & $6 \%$ & 0.49 \\
\hline
\end{tabular}

Table 186: Intervals with a positive return less than $0.10 \%$, sell orders

\begin{tabular}{|l|ccc|}
\hline & Executed order volume & Change in order volume & Price renewal \\
\hline Server type A & $40 \%$ & $110 \%$ & 2.63 \\
Server type B & $13 \%$ & $79 \%$ & 0.80 \\
Server type C & $36 \%$ & $72 \%$ & 3.22 \\
Server type D & $11 \%$ & $31 \%$ & 0.81 \\
\hline
\end{tabular}

Table 187: Intervals with a positive return greater than $0.10 \%$, buy orders

\begin{tabular}{|l|ccc|}
\hline & Executed order volume & Change in order volume & Price renewal \\
\hline Server type A & $11 \%$ & $22 \%$ & 0.83 \\
Server type B & $23 \%$ & $-42 \%$ & 0.46 \\
Server type C & $53 \%$ & $24 \%$ & 2.23 \\
Server type D & $13 \%$ & $15 \%$ & 0.42 \\
\hline
\end{tabular}

Table 188: Intervals with a positive return greater than $0.10 \%$, sell orders

\section{A.0.4 24th August}

\begin{tabular}{|l|ccc|}
\hline & Executed order volume & Change in order volume & Price renewal \\
\hline Server type A & $13 \%$ & $19 \%$ & 1.16 \\
Server type B & $27 \%$ & $-69 \%$ & 0.46 \\
Server type C & $46 \%$ & $19 \%$ & 3.44 \\
Server type D & $14 \%$ & $13 \%$ & 0.72 \\
\hline
\end{tabular}

Table 189: Intervals with a negative return greater than $0.10 \%$, buy orders

\begin{tabular}{|l|ccc|}
\hline & Executed order volume & Change in order volume & Price renewal \\
\hline Server type A & $35 \%$ & $97 \%$ & 2.50 \\
Server type B & $14 \%$ & $123 \%$ & 0.85 \\
Server type C & $40 \%$ & $76 \%$ & 5.27 \\
Server type D & $11 \%$ & $29 \%$ & 1.29 \\
\hline
\end{tabular}

Table 190: Intervals with a negative return greater than $0.10 \%$, sell orders 


\begin{tabular}{|c|ccc|}
\hline & Executed order volume & Change in order volume & Price renewal \\
\hline Server type A & $16 \%$ & $23 \%$ & 1.04 \\
Server type B & $15 \%$ & $-12 \%$ & 0.28 \\
Server type C & $54 \%$ & $33 \%$ & 3.35 \\
Server type D & $15 \%$ & $7 \%$ & 0.83 \\
\hline
\end{tabular}

Table 191: Intervals with a negative return less than $0.10 \%$, buy orders

\begin{tabular}{|l|ccc|}
\hline & Executed order volume & Change in order volume & Price renewal \\
\hline Server type A & $31 \%$ & $66 \%$ & 1.65 \\
Server type B & $12 \%$ & $49 \%$ & 0.51 \\
Server type C & $45 \%$ & $61 \%$ & 3.78 \\
Server type D & $11 \%$ & $17 \%$ & 0.85 \\
\hline
\end{tabular}

Table 192: Intervals with a negative return less than $0.10 \%$, sell orders

\begin{tabular}{|l|ccc|}
\hline & Executed order volume & Change in order volume & Price renewal \\
\hline Server type A & $34 \%$ & $78 \%$ & 1.94 \\
Server type B & $11 \%$ & $32 \%$ & 0.49 \\
Server type C & $42 \%$ & $55 \%$ & 3.99 \\
Server type D & $13 \%$ & $16 \%$ & 1.08 \\
\hline
\end{tabular}

Table 193: Intervals with a positive return less than $0.10 \%$, buy orders

\begin{tabular}{|l|ccc|}
\hline & Executed order volume & Change in order volume & Price renewal \\
\hline Server type A & $14 \%$ & $18 \%$ & 0.86 \\
Server type B & $16 \%$ & $-17 \%$ & 0.34 \\
Server type C & $57 \%$ & $38 \%$ & 3.01 \\
Server type D & $13 \%$ & $6 \%$ & 0.59 \\
\hline
\end{tabular}

Table 194: Intervals with a positive return less than $0.10 \%$, sell orders

\begin{tabular}{|c|ccc|}
\hline & Executed order volume & Change in order volume & Price renewal \\
\hline Server type A & $41 \%$ & $118 \%$ & 3.04 \\
Server type B & $13 \%$ & $95 \%$ & 0.88 \\
Server type C & $34 \%$ & $55 \%$ & 4.76 \\
Server type D & $11 \%$ & $21 \%$ & 1.18 \\
\hline
\end{tabular}

Table 195: Intervals with a positive return greater than $0.10 \%$, buy orders

\begin{tabular}{|l|ccc|}
\hline & Executed order volume & Change in order volume & Price renewal \\
\hline Server type A & $11 \%$ & $18 \%$ & 1.00 \\
Server type B & $25 \%$ & $-73 \%$ & 0.41 \\
Server type C & $52 \%$ & $23 \%$ & 3.06 \\
Server type D & $12 \%$ & $-3 \%$ & 0.56 \\
\hline
\end{tabular}

Table 196: Intervals with a positive return greater than $0.10 \%$, sell orders 


\section{A.0.5 26th August}

\begin{tabular}{|c|ccc|}
\hline & Executed order volume & Change in order volume & Price renewal \\
\hline Server type A & $16 \%$ & $30 \%$ & 2.65 \\
Server type B & $20 \%$ & $-25 \%$ & 0.82 \\
Server type C & $50 \%$ & $28 \%$ & 3.98 \\
Server type D & $14 \%$ & $10 \%$ & 1.04 \\
\hline
\end{tabular}

Table 197: Intervals with a negative return greater than $0.10 \%$, buy orders

\begin{tabular}{|c|ccc|}
\hline & Executed order volume & Change in order volume & Price renewal \\
\hline Server type A & $44 \%$ & $127 \%$ & 5.29 \\
Server type B & $18 \%$ & $81 \%$ & 1.73 \\
Server type C & $29 \%$ & $53 \%$ & 4.71 \\
Server type D & $8 \%$ & $17 \%$ & 1.15 \\
\hline
\end{tabular}

Table 198: Intervals with a negative return greater than $0.10 \%$, sell orders

\begin{tabular}{|c|ccc|}
\hline & Executed order volume & Change in order volume & Price renewal \\
\hline Server type A & $20 \%$ & $21 \%$ & 1.54 \\
Server type B & $16 \%$ & $-4 \%$ & 0.44 \\
Server type C & $50 \%$ & $28 \%$ & 2.89 \\
Server type D & $14 \%$ & $6 \%$ & 0.83 \\
\hline
\end{tabular}

Table 199: Intervals with a negative return less than $0.10 \%$, buy orders

\begin{tabular}{|l|ccc|}
\hline & Executed order volume & Change in order volume & Price renewal \\
\hline Server type A & $37 \%$ & $77 \%$ & 2.72 \\
Server type B & $16 \%$ & $30 \%$ & 0.85 \\
Server type C & $38 \%$ & $43 \%$ & 2.99 \\
Server type D & $9 \%$ & $14 \%$ & 0.62 \\
\hline
\end{tabular}

Table 200: Intervals with a negative return less than $0.10 \%$, sell orders

\begin{tabular}{|c|ccc|}
\hline & Executed order volume & Change in order volume & Price renewal \\
\hline Server type A & $34 \%$ & $75 \%$ & 2.19 \\
Server type B & $14 \%$ & $38 \%$ & 0.58 \\
Server type C & $41 \%$ & $54 \%$ & 3.14 \\
Server type D & $12 \%$ & $15 \%$ & 0.94 \\
\hline
\end{tabular}

Table 201: Intervals with a positive return less than $0.10 \%$, buy orders

\begin{tabular}{|l|ccc|}
\hline & Executed order volume & Change in order volume & Price renewal \\
\hline Server type A & $22 \%$ & $21 \%$ & 1.65 \\
Server type B & $17 \%$ & $-10 \%$ & 0.53 \\
Server type C & $49 \%$ & $28 \%$ & 2.17 \\
Server type D & $12 \%$ & $6 \%$ & 0.41 \\
\hline
\end{tabular}

Table 202: Intervals with a positive return less than $0.10 \%$, sell orders 


\begin{tabular}{|c|ccc|}
\hline & Executed order volume & Change in order volume & Price renewal \\
\hline Server type A & $40 \%$ & $119 \%$ & 4.31 \\
Server type B & $14 \%$ & $93 \%$ & 1.29 \\
Server type C & $35 \%$ & $68 \%$ & 5.24 \\
Server type D & $11 \%$ & $28 \%$ & 1.67 \\
\hline
\end{tabular}

Table 203: Intervals with a positive return greater than $0.10 \%$, buy orders

\begin{tabular}{|l|ccc|}
\hline & Executed order volume & Change in order volume & Price renewal \\
\hline Server type A & $18 \%$ & $31 \%$ & 2.61 \\
Server type B & $24 \%$ & $-50 \%$ & 0.95 \\
Server type C & $45 \%$ & $23 \%$ & 3.19 \\
Server type D & $12 \%$ & $3 \%$ & 0.57 \\
\hline
\end{tabular}

Table 204: Intervals with a positive return greater than $0.10 \%$, sell orders

\section{A.0.6 8th July}

\begin{tabular}{|l|ccc|}
\hline & Executed order volume & Change in order volume & Price renewal \\
\hline Server type A & $11 \%$ & $17 \%$ & 0.52 \\
Server type B & $21 \%$ & $-52 \%$ & 0.31 \\
Server type C & $50 \%$ & $29 \%$ & 2.52 \\
Server type D & $18 \%$ & $24 \%$ & 0.81 \\
\hline
\end{tabular}

Table 205: Intervals with a negative return greater than $0.10 \%$, buy orders

\begin{tabular}{|l|ccc|}
\hline & Executed order volume & Change in order volume & Price renewal \\
\hline Server type A & $31 \%$ & $109 \%$ & 1.91 \\
Server type B & $14 \%$ & $270 \%$ & 0.75 \\
Server type C & $39 \%$ & $89 \%$ & 4.26 \\
Server type D & $16 \%$ & $31 \%$ & 1.10 \\
\hline
\end{tabular}

Table 206: Intervals with a negative return greater than $0.10 \%$, sell orders

\begin{tabular}{|l|ccc|}
\hline & Executed order volume & Change in order volume & Price renewal \\
\hline Server type A & $15 \%$ & $22 \%$ & 0.51 \\
Server type B & $14 \%$ & $-19 \%$ & 0.19 \\
Server type C & $54 \%$ & $34 \%$ & 1.93 \\
Server type D & $16 \%$ & $11 \%$ & 0.51 \\
\hline
\end{tabular}

Table 207: Intervals with a negative return less than $0.10 \%$, buy orders

\begin{tabular}{|l|ccc|}
\hline & Executed order volume & Change in order volume & Price renewal \\
\hline Server type A & $31 \%$ & $90 \%$ & 1.17 \\
Server type B & $13 \%$ & $89 \%$ & 0.42 \\
Server type C & $43 \%$ & $75 \%$ & 2.44 \\
Server type D & $13 \%$ & $27 \%$ & 0.62 \\
\hline
\end{tabular}

Table 208: Intervals with a negative return less than $0.10 \%$, sell orders 


\begin{tabular}{|c|ccc|}
\hline & Executed order volume & Change in order volume & Price renewal \\
\hline Server type A & $36 \%$ & $109 \%$ & 1.24 \\
Server type B & $10 \%$ & $42 \%$ & 0.30 \\
Server type C & $41 \%$ & $60 \%$ & 2.34 \\
Server type D & $14 \%$ & $29 \%$ & 0.69 \\
\hline
\end{tabular}

Table 209: Intervals with a positive return less than $0.10 \%$, buy orders

\begin{tabular}{|l|ccc|}
\hline & Executed order volume & Change in order volume & Price renewal \\
\hline Server type A & $13 \%$ & $17 \%$ & 0.43 \\
Server type B & $19 \%$ & $104 \%$ & 0.21 \\
Server type C & $55 \%$ & $44 \%$ & 1.64 \\
Server type D & $13 \%$ & $5 \%$ & 0.40 \\
\hline
\end{tabular}

Table 210: Intervals with a positive return less than $0.10 \%$, sell orders

\begin{tabular}{|l|ccc|}
\hline & Executed order volume & Change in order volume & Price renewal \\
\hline Server type A & $38 \%$ & $149 \%$ & 1.79 \\
Server type B & $11 \%$ & $94 \%$ & 0.55 \\
Server type C & $37 \%$ & $66 \%$ & 6.40 \\
Server type D & $14 \%$ & $35 \%$ & 1.79 \\
\hline
\end{tabular}

Table 211: Intervals with a positive return greater than $0.10 \%$, buy orders

\begin{tabular}{|l|ccc|}
\hline & Executed order volume & Change in order volume & Price renewal \\
\hline Server type A & $9 \%$ & $13 \%$ & 0.36 \\
Server type B & $29 \%$ & $-63 \%$ & 0.31 \\
Server type C & $49 \%$ & $44 \%$ & 1.54 \\
Server type D & $14 \%$ & $1 \%$ & 0.36 \\
\hline
\end{tabular}

Table 212: Intervals with a positive return greater than $0.10 \%$, sell orders

\section{A.0.7 9th July}

\begin{tabular}{|l|ccc|}
\hline & Executed order volume & Change in order volume & Price renewal \\
\hline Server type A & $17 \%$ & $23 \%$ & 1.18 \\
Server type B & $25 \%$ & $-41 \%$ & 0.42 \\
Server type C & $45 \%$ & $29 \%$ & 1.84 \\
Server type D & $13 \%$ & $8 \%$ & 0.39 \\
\hline
\end{tabular}

Table 213: Intervals with a negative return greater than $0.10 \%$, buy orders

\begin{tabular}{|l|ccc|}
\hline & Executed order volume & Change in order volume & Price renewal \\
\hline Server type A & $28 \%$ & $95 \%$ & 2.02 \\
Server type B & $14 \%$ & $97 \%$ & 0.91 \\
Server type C & $45 \%$ & $75 \%$ & 3.83 \\
Server type D & $13 \%$ & $24 \%$ & 1.20 \\
\hline
\end{tabular}

Table 214: Intervals with a negative return greater than $0.10 \%$, sell orders 


\begin{tabular}{|c|ccc|}
\hline & Executed order volume & Change in order volume & Price renewal \\
\hline Server type A & $17 \%$ & $29 \%$ & 0.73 \\
Server type B & $15 \%$ & $-20 \%$ & 0.24 \\
Server type C & $54 \%$ & $43 \%$ & 1.54 \\
Server type D & $14 \%$ & $5 \%$ & 0.34 \\
\hline
\end{tabular}

Table 215: Intervals with a negative return less than $0.10 \%$, buy orders

\begin{tabular}{|l|ccc|}
\hline & Executed order volume & Change in order volume & Price renewal \\
\hline Server type A & $30 \%$ & $100 \%$ & 1.12 \\
Server type B & $12 \%$ & $45 \%$ & 0.47 \\
Server type C & $47 \%$ & $75 \%$ & 2.67 \\
Server type D & $11 \%$ & $16 \%$ & 0.65 \\
\hline
\end{tabular}

Table 216: Intervals with a negative return less than $0.10 \%$, sell orders

\begin{tabular}{|l|ccc|}
\hline & Executed order volume & Change in order volume & Price renewal \\
\hline Server type A & $37 \%$ & $94 \%$ & 1.71 \\
Server type B & $11 \%$ & $42 \%$ & 0.44 \\
Server type C & $42 \%$ & $71 \%$ & 2.27 \\
Server type D & $11 \%$ & $24 \%$ & 0.53 \\
\hline
\end{tabular}

Table 217: Intervals with a positive return less than $0.10 \%$, buy orders

\begin{tabular}{|l|ccc|}
\hline & Executed order volume & Change in order volume & Price renewal \\
\hline Server type A & $15 \%$ & $22 \%$ & 0.61 \\
Server type B & $15 \%$ & $-14 \%$ & 0.27 \\
Server type C & $57 \%$ & $33 \%$ & 2.08 \\
Server type D & $13 \%$ & $6 \%$ & 0.49 \\
\hline
\end{tabular}

Table 218: Intervals with a positive return less than $0.10 \%$, sell orders

\begin{tabular}{|l|ccc|}
\hline & Executed order volume & Change in order volume & Price renewal \\
\hline Server type A & $40 \%$ & $110 \%$ & 2.63 \\
Server type B & $13 \%$ & $79 \%$ & 0.80 \\
Server type C & $36 \%$ & $72 \%$ & 3.22 \\
Server type D & $11 \%$ & $31 \%$ & 0.81 \\
\hline
\end{tabular}

Table 219: Intervals with a positive return greater than $0.10 \%$, buy orders

\begin{tabular}{|l|ccc|}
\hline & Executed order volume & Change in order volume & Price renewal \\
\hline Server type A & $11 \%$ & $22 \%$ & 0.83 \\
Server type B & $23 \%$ & $-42 \%$ & 0.46 \\
Server type C & $53 \%$ & $24 \%$ & 2.23 \\
Server type D & $13 \%$ & $15 \%$ & 0.42 \\
\hline
\end{tabular}

Table 220: Intervals with a positive return greater than $0.10 \%$, sell orders 